\title{
Unique PGE-Cu-Ni Noril'sk Deposits, Siberian Trap Province: Magmatic and Tectonic Factors in Their Origin
}

\author{
Nadezhda A. Krivolutskaya $1, *\left(\mathbb{D}\right.$, Anton V. Latyshev ${ }^{2,3}$, Alexander S. Dolgal ${ }^{4}$, \\ Bronislav I. Gongalsky ${ }^{5}$, Elena M. Makarieva ${ }^{6}$, Alexander A. Makariev ${ }^{6}$, Natalia M. Svirskaya ${ }^{1}$, \\ Yana V. Bychkova ${ }^{2}$, Anton I. Yakushev ${ }^{5}$ and Alexey M. Asavin ${ }^{1}$ \\ 1 Vernadsky Institute of Geochemistry and Analytical Chemistry, Russian Academy of Sciences, \\ 19 Kosygin st., Moscow 119991, Russia; nsvirsk@mail.ru (N.M.S.); aalex06@inbox.ru (A.M.A.) \\ 2 Geological Department, Lomonosov Moscow State University, Leninsky Gory, 1, Moscow 119992, Russia; \\ anton.latyshev@gmail.com (A.V.L.); yanab66@yandex.ru (Y.V.B.) \\ 3 Schmidt Institute of the Physics of the Earth, Russian Academy of Sciences, 10 Bolshaya Gruzinskaya, \\ Moscow 123995, Russia \\ 4 Geological Department, Perm State University, 15 Bukireva, Perm 614990, Russia; Dolgal@mi-perm.ru \\ 5 Institute of Geology of Ore Deposits, Petrography, Mineralogy and Geochemistry, \\ Russian Academy of Sciences, 35 Staronometny per., Moscow 119017, Russia; brgon@mail.ru (B.I.G.); \\ antemp@inbox.ru (A.I.Y.) \\ 6 Polyarnaya Expedition, Lomonosov, 24, Pobedy str., Lomonosov, St.-Petersburg 198412, Russia; \\ makareva@mail.ru (E.M.M.); almakarev@mail.ru (A.A.M.) \\ * Correspondence: nakriv@mail.ru; Tel.: +7-495-93 9-70-17
}

Received: 11 December 2018; Accepted: 18 January 2019; Published: 21 January 2019

\begin{abstract}
The unique and very large PGE-Cu-Ni Noril'sk deposits are located within the Siberian trap province, posing a number of questions about the relationship between the ore-forming process and the magmatism that produced the traps. A successful answer to these questions could greatly increase the possibility of discovering new deposits in flood basalt provinces elsewhere. In this contribution, we present new data on volcanic stratigraphy and geochemistry of the magmatic rocks in the key regions of the Siberian trap province (Noril'sk, Taimyr, Maymecha-Kotuy, Kulyumber, Lower Tunguska and Angara) and analyze the structure of the north part of the province. The magmatic rocks of the Arctic zone are characterized by variable $\mathrm{MgO}(3.6-37.2 \mathrm{wt} \%)$ and $\mathrm{TiO}_{2}(0.8-3.9 \mathrm{wt} \%)$ contents, $\mathrm{Gd} / \mathrm{Yb}(1.4-6.3)$ and $\mathrm{La} / \mathrm{Sm}(2.0-10.4)$ ratios, and a large range of isotopic compositions. The intrusions in the center of the Tunguska syneclise and Angara syncline have much less variable compositions and correspond to a "typical trap" with $\mathrm{MgO}$ of 5.6-7.2 wt \%, $\mathrm{TiO}_{2}$ of 1.0-1.6 wt \%, $\mathrm{Gd} / \mathrm{Yb}$ ratio of 1.4-1.6 and $\mathrm{La} / \mathrm{Sm}$ ratio of 2.0-3.5. This compositional diversity of magmas in the Arctic zone is consistent with their emplacement within the paleo-rift zones. Ore-bearing intrusions (the Noril'sk 1, Talnakh, Kharaelakh) are deep-situated in the Igarka-Noril'sk rift zone, which has three branches, namely the Bolsheavamsky, Dyupkunsky, and Lower Tunguska, that are prospected for discovering new deposits. One possible explanation for the specific position of the PGE-Cu-Ni deposits is accumulation of sulfides in these long-lived zones from the Neoproterozoic to the Mesozoic era during magmatic and metamorphic processes. Thus, trap magmatism, itself, does not produce large deposits, but mobilizes earlier formed sulfide segregations in addition carrying metals in the original magmas. These deposits are the results of several successive magmatic events, in which emplacement of the traps was the final event.
\end{abstract}

Keywords: Siberian traps; magmatism; geochemistry; PGE-Cu-Ni deposits; paleo-rift 


\section{Introduction}

The Noril'sk deposits play a leading role in the economics of the world's supply of $\mathrm{Ni}$ and PGE. They are unique among PGE-Cu-Ni deposits around the world due to their location inside the Phanerozoic Siberian traps province of continental flood basalts [1,2], in contrast to most deposits which are related to large Proterozoic layered intrusions. The Noril'sk giant bodies of massive sulfide ore (up to $54 \mathrm{~m}$ ) extremely enriched in PGE are related to thin (100-150 m) subvolcanic basic intrusions. Despite the abundance of basic intrusions in the Noril'sk area, only three massifs contain economically important PGE-Cu-Ni-rich ores (the Talnakh, Kharaelakh, and Noril'sk 1), whereas many other massifs contain only poor mineralization (e.g., Zub-Marksheydersky, Bolshaya Bar'ernaya, and Mikchangdinsky). At the same time, thousands of barren intrusive bodies are known not only in the Noril'sk region but also over the entire Siberian province.

The location of the intrusions within the Siberian trap province and the geochemical similarities between the intrusions and lavas pose a question about the possible relationships between magmatic and ore-forming processes in this area. Were the deposits formed under special conditions, or was their origin predetermined by the evolution of the trap system as a whole? Could we expect the discovery of large $\mathrm{Cu}-\mathrm{Ni}$ deposits in flood-basalt provinces elsewhere? To answer these questions, it is necessary to analyze the structure of the Siberian province and the evolution of magmatism within it.

The tectonic positions of intrusions are rarely taken into account in genetic models of the Noril'sk deposits with the most authors focusing on their mineralogy and geochemistry. There are two widespread models of the sulfide ore origin in the Noril'sk area suggesting ore formation either in closed or in open magmatic system. The first hypothesis suggests that the sulfides were transported by magma from deep zones of the crust or mantle into the shallow chamber where they formed a disseminated and massive ore [3-9]. The second model proposes the sulfide formation in situ due to the long-term flow of magma to the surface and its interactions with surrounding rocks, in particular, the evaporates that provided a source of sulfur [10-13]. According to this model, ore-bearing intrusions are horizontal parts of the magma conduits to the surface. Merits and imperfections of this model are considered in [14]. In connection with the subject of this article, it is important to note that based on the suggestion of an open magmatic system, sulfide deposits could have been formed everywhere within the trap province, especially in the south part, where evaporates are abundant [15]. The problem of surrounding rocks' assimilation has been discussed by us earlier [14]. Nevertheless, the ore deposits are located only in the NW part of the province.

Ore crystallization in a closed magmatic system attaches great importance to both magmatic and tectonic factors for the formation of deposits. Many publications have demonstrated that these deposits are related only to high-Mg magma (10-12 wt \% MgO) widespread in the NW part of the Siberian traps province. This model suggests the sulfide transportation by magmas as small drops from the mantle and their segregation in a modern chamber $[5,7,16]$. Based on geophysical data, the localization of high-Mg magmas in the limited area within the province could reflect the presence of deep faults along which mantle magma reached the upper crust, where it was differentiated and sulfide separation occurred in intermediate chambers [17-19].

New data on magmatism and tectonics in the Siberian platform obtained during recent decades has forced a re-evaluation of models for the Noril'sk deposits origin. First, it was shown that deep, mantle-tapping occurs in the central region of the Siberian craton, whereas many faults in the Noril'sk area, including the main Noril'sk-Kharaelakh fault, do not penetrate the mantle [20-22]. Second, the chemical compositions of the PGE-Cu-Ni mineralized intrusions have lower crustal signatures according to Rudnick and Gao data [23] that was shown in the articles [24-27], and, consequently, the parental magma for these intrusions could not be derived directly from the mantle. These new data require a new interpretation. Here we discuss only one aspect of the geology of the Noril'sk deposits, namely, their location in the northwest part of the Siberian trap province and their absence from the reminder of the province. 
Obviously, without considering this fact, any models of deposits origin are untenable. We demonstrate that the evolution of both effusive and intrusive magmatism in the north part of the trap province hosting the Noril'sk deposits differs from its central and south parts due to different structures $[19,28,29]$. The tectonics positions (inside the rift zones or the stable platform) and the conditions of magma origin were of fundamental importance for the formation of these deposits. Ore-bearing intrusions were formed only in long-developing rift zones, where the chemical composition of magma also had its own specific features.

Analyses of potential relationships between ore-bearing intrusions and trap magmatism must include (i) the evolution of the volcanism itself and (ii) the subdivision of intrusions into complexes, their correlations with volcanic formations, and distribution within the province. To analyze these relationships we used our new geochemical, paleomagnetic, and geophysical data from the key areas of the Siberian trap province. In particular, we present new geochemical data on volcanic and intrusive rocks from throughout the province. We suggest a new version of the Noril'sk-deposit origin model based on published and new data obtained from volcanic and intrusive rocks during the last several years. The sulfides of the Noril'sk deposits accumulated in multiple stages during several geological events from the Proterozoic until the Mesozoic era rather than during final magma emplacement.

\section{Materials and Methods}

The authors studied magmatic rocks in the key parts of the Siberian trap province, including (Figure 1a,b) the Noril'sk area (1), the Taimyr peninsula (2), the Maymecha-Kotuy area (3), the valleys of the Kulyumber river (or Kulumbe in some publications) (4), the Lower (Nizhnyaya) Tunguska river (5), and the Angara river (or Angara-Taseeva syncline) (6). Samples (Supplementary Materials) were mainly taken from outcrops and from some borehole cores in the Noril'sk region.

Major element concentrations in rocks were analyzed using X-ray fluorescence (XRF) at the Institute Geology of Ore Deposits, Petrography, Mineralogy and Geochemistry RAS on a sequenced-action vacuum spectrometer (with dispersion over the wavelength), and using an Axios mAX model from PANalytical (Almelo, The Netherlands). This spectrometer is equipped with a $4-\mathrm{kW}$ X-ray tube with a Rh-anode. The maximum voltage on the tube is $60 \mathrm{kV}$, and the maximum anode current is $160 \mathrm{~mA}$. When the spectrometer is calibrated, the industry and state standard samples of the chemical composition of rocks and ores are used. The quality control of the results is carried out according to the analysis of rock standard samples from the US Geological Survey (USGS) [30]. The relative standard deviations (RSD) for major oxides are as follows (\%): $\mathrm{SiO}_{2}-0.12$; $\mathrm{TiO}_{2}-0.05 ; \mathrm{Al}_{2} \mathrm{O}_{3}-0.12 ; \mathrm{Fe}_{2} \mathrm{O}_{3 \text { (total) }}-0.13 ; \mathrm{MnO}-0.005 ; \mathrm{MgO}-0.07 ; \mathrm{CaO}-0.08 ; \mathrm{Na}_{2} \mathrm{O}-0.08$; $\mathrm{K}_{2} \mathrm{O}-0.03$; and $\mathrm{P}_{2} \mathrm{O}_{5}-0.01$.

Data on the distribution of rare elements in volcanic and intrusive rocks were obtained using inductively coupled plasma mass spectrometry (ICP-MS) at Lomonosov Moscow State University. For these analyses, $100 \mathrm{mg}$ of powdered rock sample was sintered with $\mathrm{Na}_{2} \mathrm{CO}_{3}(1: 3)$ in a muffle furnace at a temperature of $800^{\circ} \mathrm{C}$ for $2 \mathrm{~h}$; it was then dissolved in $3.5 \mathrm{~mL} \mathrm{HNO3-HF} \mathrm{(10:1)} \mathrm{with} 0.5 \mathrm{~mL} \mathrm{HCl}$ and diluted by $50 \mathrm{~mL} 3 \% \mathrm{HNO}_{3}$. Prior to analysis, the samples were diluted 100 -fold in $3 \% \mathrm{HNO}_{3}$.

Trace elements were measured in low and medium resolution on the sector field double-focusing ELEMENT-2 ICP-MS. Analyses were monitored using indium as an internal standard, and data quality was monitored using USGS reference materials (DTS-1, BHVO-2, BCR-2). The relative standard deviations (RSD) for elements are as follows (\%): $\mathrm{La}-1 ; \mathrm{Rb}, \mathrm{Sr}, \mathrm{Y}, \mathrm{Ba}, \mathrm{Ce}, \mathrm{Pr}, \mathrm{Nd}, \mathrm{Er}, \mathrm{Th}, \mathrm{U}, \mathrm{Pb}, \mathrm{Ta}, \mathrm{Nb}$, $\mathrm{Zr}-2$; Sm, Eu, Gd, Tb, Dy, Ho, Yb, Hf, Sc, Co, Ni, Cu, Zn- 3; Lu, Tm-4. 

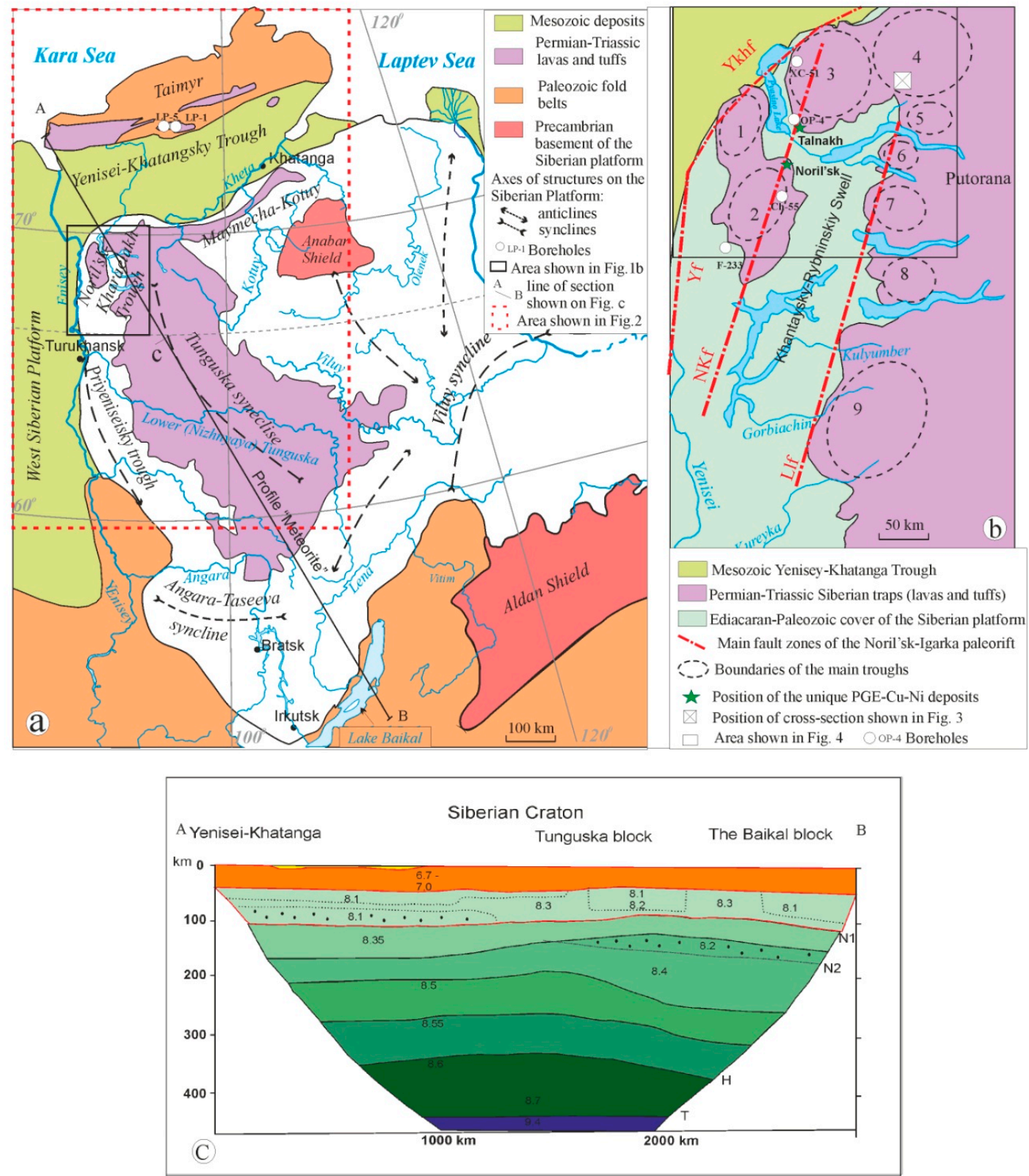

Figure 1. Tectonic schema of the Siberian platform and adjacent fold belts (a), schema of the Northwest Siberian platform (b) and velocity cross-section along the "Meteorite" seismic profile (c) (after [22]). Fault zones (Figure 1b): NKf-Noril'sk-Kharaelakh; LLf-Lamsko-Letninskiy; Yf-Yeniseysky, YKhfYenisey-Khatanga. The main troughs of the Siberian Traps: 1-Vologochan; 2-Noril'sk; 3-Kharaelakh; 4-Iken; 5-Mikchangda; 6-Lama; 7-Imangda; 8-Khantayka; 9-Kureyka. Figure 1c: The seismic boundaries N1, N2, H are the upper mantle basic boundaries with constant velocity of longitudinal waves (the velocity increases linearly between the boundaries); T-boundary is the top of the upper/lower mantle transition zone. $\mathrm{Vr}=8.1 \mathrm{~km} / \mathrm{s}$.

\section{Geological Background}

The Siberian trap province covering 1.5 million $\mathrm{km}^{2}$ [31], is the largest continental flood basalt province on Earth. Siberian traps are located in the central part of the East Siberian platform and the South Taimyr peninsula (Figure 1a); these were combined into a single craton at the end 
of the Proterozoic [32]. The main structures are (Figure 1a): (1) the Anabar and Aldan shields, (2) the Tunguska, Viluy, and Angara-Taseeva syneclises, and (3) the Yenisey-Khatanga, Priyeniseysky, and Noril'sk-Kharaelakh troughs [19]. The interpretation of the deep structure of the Siberian Platform [22] is based on a number of seismic profiles (that were constructed with the aid of nuclear and chemical explosions, methods of deep seismic sounding). The main seismic profiles are "Craton" and "Kimberlite", across the West Siberian and East Siberian platforms, and the "Meteorite" profile from Taimyr to Baikal (Figure 1c). The latter shows a clear difference in the structure of the lithosphere under the Yenisey-Khatanga trough and the Angara-Taseeva syneclise in comparison with the Siberian Craton. Low velocity zones (velocity of longitudinal waves is $8.1 \mathrm{~km} / \mathrm{s}$ ) occur at a depth of $80-100 \mathrm{~km}$ under the Yenisey-Khatanga trough, and 110-160 km under the Siberian craton. Based on these data, as well as large-scale geophysical data, there is also a significant difference in the crustal structure of the north and central parts of the province is shown as well (Figure 1c). The Moho boundary rises to a depth of $31-32 \mathrm{~km}$ in the Norilsk region while it is located at $51 \mathrm{~km}$ in the center of the Tunguska syncline.

The rocks of the crystalline basement $\left(\mathrm{AR}, \mathrm{PR}_{1}\right)$ are exposed on the surface within the Aldan and Anabar shields (Figure 2a). The platform cover complex is composed of deep- and shallow-water marine sedimentary rocks from the Ediacaran to the Carboniferous (with evaporates and salts) (Figure 2a), that are overlapped by clastic rocks of the Tunguska Group (with interlayers of coal, $\left.\mathrm{C}_{2}-\mathrm{P}_{2}\right)$ and trap volcanic rocks $\left(\mathrm{P}_{3}-\mathrm{T}_{1}\right)$. Intrusive rocks of the Siberian trap province were subdivided into the different complexes based on their internal structure and composition. As a result of post-Mesozoic structural transformations and erosion processes, many dolerite sills that occur in Paleozoic sedimentary rocks are now exposed on the surface (Figure 2a). The data of deep seismic sounding on super-long profiles, taking into account the magnetometric and gravimetric mapping at 1:200,000 scale, as well as magnetotelluric sounding, allowed the distinction of three layers in the structure of the crust of the Siberian platform and to calculation of the coefficient of the crust basification (defined as the proportion of the volume of basic and ultrabasic rocks to the whole volume of the crust). Based on these data Erinchek [33] constructed the map of the Siberian trap province (Figure $2 b$ ) which demonstrates the significant difference between its north and south parts. The maximum saturation of the crust with basic and ultrabasic rocks is typical of the Yenisey-Khatanga trough [32]. Masaitis [28] and Zolotukhin et al. [31] subdivided the province into three large zones: Zone I, the north-central zone, is mainly represented by lavas; Zone II, the south zone consists of tuffs, and Zone III is the border zones, where intrusive bodies are located in sedimentary rocks (Figure 2a). The north part of the province within and adjacent to the Yenisey-Khatanga trough is characterized by the maximal thickness of volcanic sections and diversity of the igneous rocks compositions, which differs significantly from the other parts of the province. This has been confirmed by drilling and geophysical (e.g., gravity, magnetic) data [18]. Volcanic rocks have been subdivided into different formations in different parts of the province (Table 1). Their correlation schemes are suggested in some publications [31,34-36]. These detailed works were mainly based on the major components of these rocks and did not include trace elements and isotope data. Precious geochemical data from magmatic rocks were later obtained and covered only two areas (Noril'sk and Maymecha-Kotuy, Figure 2b; [37-41]). Intrusive rocks are well studied only in the Noril'sk area due to their unique mineralization. They have been described quite well $[4,6,7,42-46]$. In contrast, intrusions from other parts of the Siberian platform were studied many years ago [34,47-50] and have hardly been analyzed using modern geochemical methods.

\subsection{Volcanic Rocks of the Siberian Traps Province}

Urvantsev [51] discovered PGE-Cu-Ni deposits in the north of East Siberia at the beginning of the 20th century (1920-1926 in the Noril'sk area and 1929 in the Taimyr); the relationship between deposits and the volcanic rocks was noted at the same time. But only after regional geological studies around the Siberian trap province it became clear that the deposits are localized only in its north part. This 
area was identified as the North-Siberian nickel-bearing province [52] characterized by maximum thickness and diversity of volcanic rocks. Because the intrusions are mostly covered by sedimentary and volcanic rocks it is very difficult to estimate their composition and determine if they contain mineralization. Only volcanic rocks exposed on the surface give complete information on the potential occurrence of intrusions in the area due to their common magma sources with plutonic rocks. The volcanic rocks carry basic information about magmatism in the area, so their study is important for our understanding of the magmatic evolution. Preliminary data show two types of regimes in the north Siberian trap province where PGE-Cu-Ni deposits found: earlier rift and later platform stages [53]. We demonstrate that these regimes existed simultaneously during the beginning stage of magmatism.

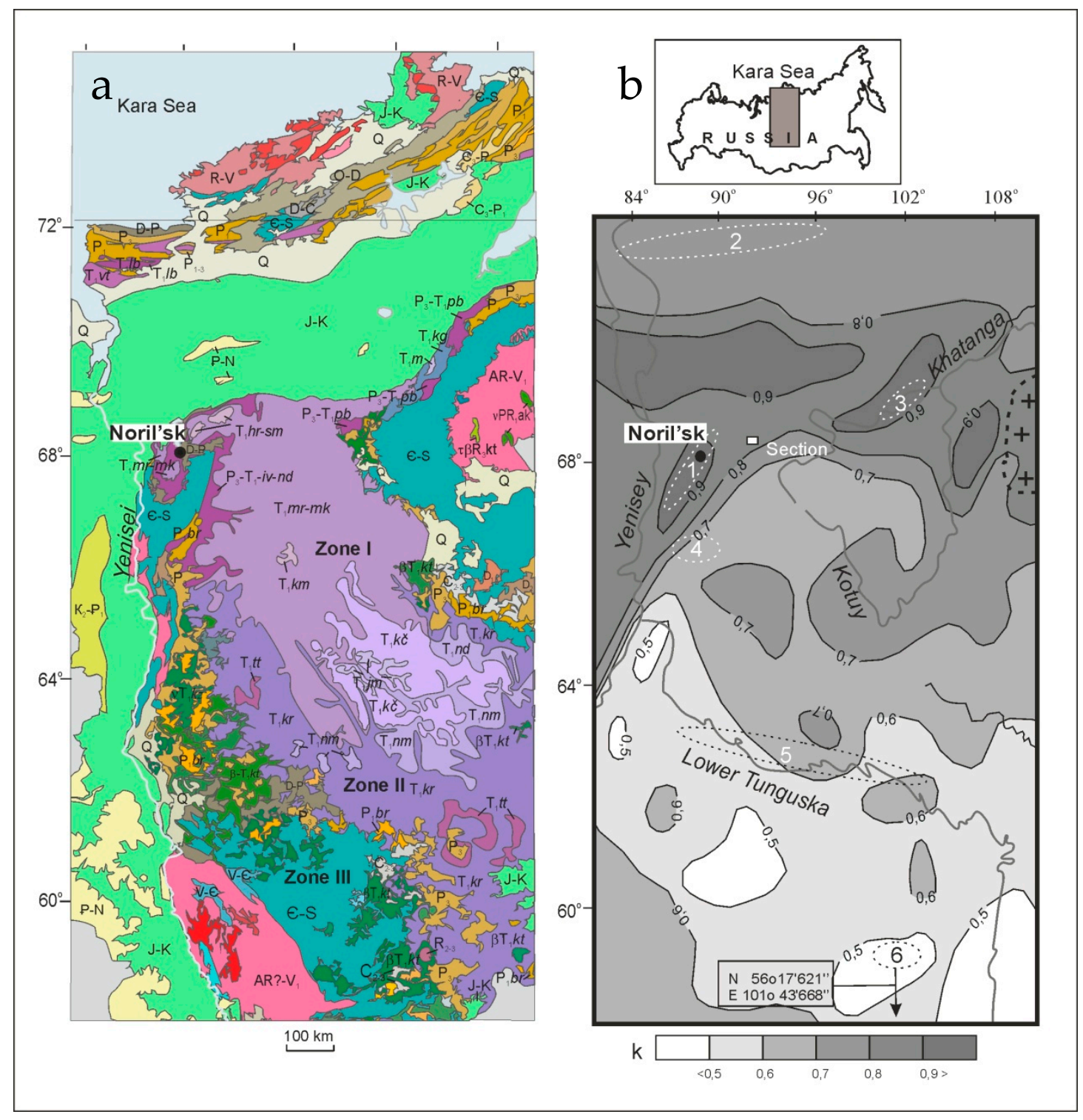

Figure 2. Cont. 


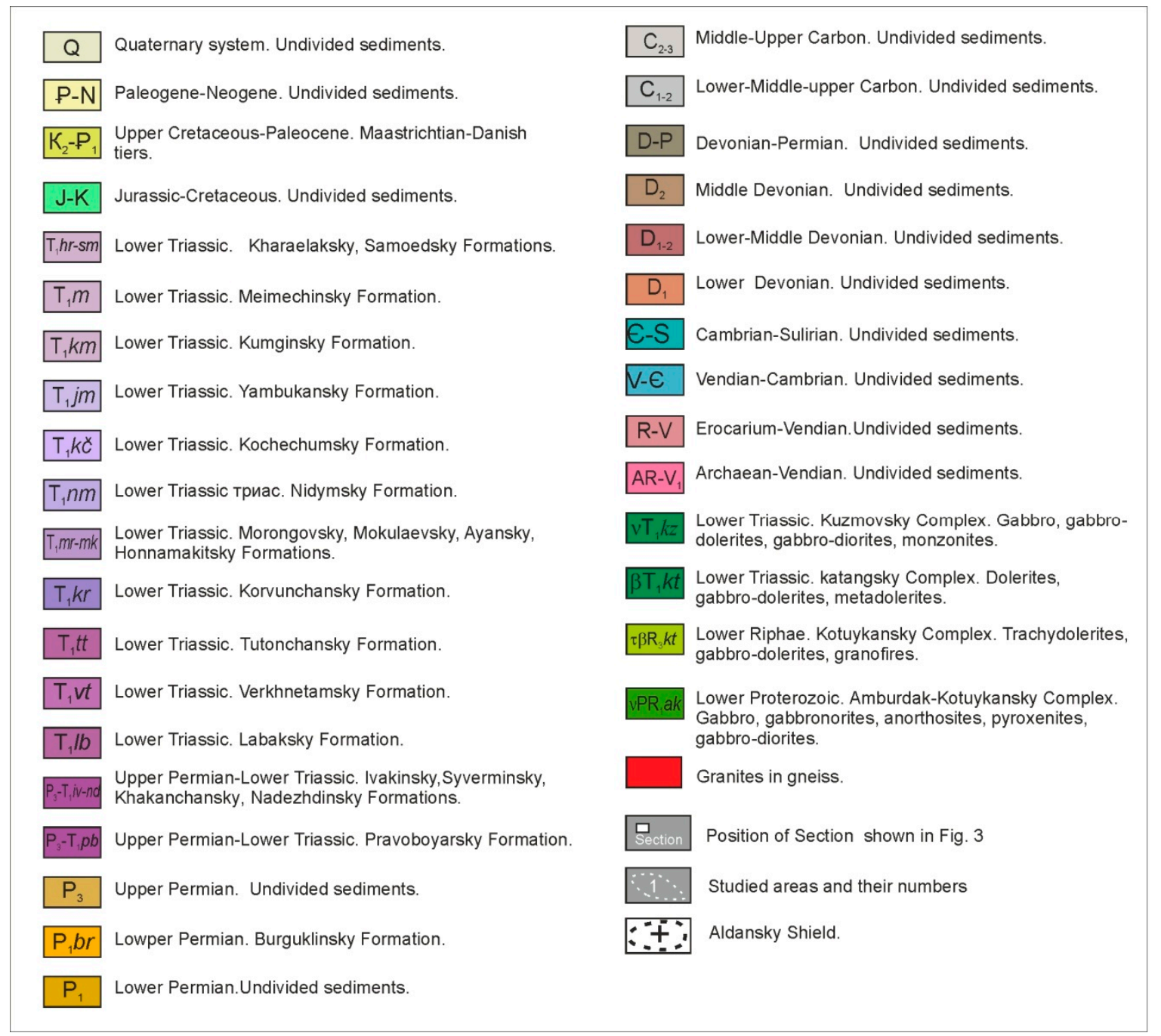

Figure 2. (a) Geological map of the Krasnoyarsk district modified after [36] and (b) map of the k-coefficient basement for the crust (defined as the ratio of the thickness of mafic and ultramafic rocks to the whole thickness of the crust) modified after [33]. The numbers on Figure $2 b$ indicate the areas studied in this project: 1-Noril'sk, 2-Taimyr, 3-Maymecha-Kotuy, 4-r. Kulyumber, 5-r. Lower Tunguska, 6-r. Angara (outside of the map, with coordinates). The position of the stratigraphic section given in Figure 3 is labeled as "Section".

The volcanic rocks of the Siberian trap province were subdivided into different formations in different areas. We give the list of formations and their roughcorrelation based on data [31,54] in Table 1. Here, we discuss their characteristics successively from north to south in six studied locations shown in Figure 2b.

The Noril'sk area (" 1 " in Figure $2 \mathrm{~b}$ ) has been studied in more detail due to its huge thickness of volcanic rocks and the presence of world-class ore deposits in this region. Russian geologists developed a stratigraphic subdivision of the Noril'sk volcanic rocks in the 1960s during a regional-scale geological survey [55-57]. Eleven formations form two groups: (1) the high-Ti $\left(\mathrm{TiO}_{2}>1.5-3 \mathrm{wt} \%\right.$ and high $\mathrm{Gd} / \mathrm{Yb}$ ratios of $>2)$ subalkaline basalt of the Ivakinsky $\left(\mathrm{P}_{3} \mathrm{iv}\right)$ and Syverminsky $\left(\mathrm{T}_{1} \mathrm{sv}\right)$ formations and the basalt and picritic basalt of the Gudchikhinsky formation ( $\mathrm{T}_{1} \mathrm{gd}$ ); and (2) the low-Ti tholeiitic basalt $\left(\mathrm{TiO}_{2} \leq 1.5 \mathrm{wt} \%\right.$, with low $\mathrm{Gd} / \mathrm{Yb}$ ratios of $\left.<2\right)$ of the Khakanchansky $\left(\mathrm{T}_{1}\right.$ hk), Tuklonsky $\left(\mathrm{T}_{1} \mathrm{tk}\right)$, Nadezhdinsky ( $\left.\mathrm{T}_{1} \mathrm{nd}\right)$, Morongonvsky $\left(\mathrm{T}_{1} \mathrm{mr}\right)$, Mokulaevsky $\left(\mathrm{T}_{1} \mathrm{mk}\right)$, Kharaelakhsky $\left(\mathrm{T}_{1} \mathrm{hr}\right)$, Kumginsky $\left(\mathrm{T}_{1} \mathrm{~km}\right)$, and Samoedsky $\left(\mathrm{T}_{1} \mathrm{sm}\right)$ formations. 
Table 1. Schema of volcanic formations within the Siberian trap province.

\begin{tabular}{|c|c|c|c|c|c|}
\hline System & Noril'sk & Taimyr & Putorana & Tunguska & Maymecha-Kotuy \\
\hline \multirow{10}{*}{$\mathrm{T}_{1}$} & & Lavas: & \multirow{11}{*}{$\begin{array}{c}\text { Lavas: } \\
\text { Nerakarsky } \\
\text { Honnamakitskyy } \\
\text { Ayansky }\end{array}$} & & \\
\hline & Lavas: Samoedsky & Fadiykudinsky & & Lavas: Yambukansky & Lavas: Maymechinsky \\
\hline & Kumginsky & Ayatarinsky & & Kochechumsky & Delkansky \\
\hline & Kharaelakhsky & & & Nidymsky & Tyvankitsky/Onkuchaksky/ \\
\hline & Mokulaevsky & Lavas: & & & Arydzhangsky \\
\hline & Morongovsky & Betlingsky & & & \\
\hline & Nadezhdinsky & Zverinsky & & & \\
\hline & Tuklonsky (Tuffs) & Lavas: & & Tuffs. Koryunchansky & Tuffs. Prayohovarcky \\
\hline & Khakanchansky & Labaksky & & $\begin{array}{l}\text { Tutts: Korvunchansky } \\
\text { Tutonchansky }\end{array}$ & $\begin{array}{c}\text { Tuffs: Pravoboyarsky } \\
\text { Khardakhsky }\end{array}$ \\
\hline & $\begin{array}{l}\text { Gudchikhinsky } \\
\text { Syverminsky }\end{array}$ & Verkhnetamsky & & & \\
\hline $\mathrm{P}_{3}$ & Lavas: Ivakinsky & Lavas: Syradasaysky & & $\begin{array}{l}\text { Tuffs: Degalinsky } \\
\text { Pelyatkinsky }\end{array}$ & Tuffs: Pelyatkinsky \\
\hline
\end{tabular}


The first group of volcanic rocks in the Noril'sk area was assigned to the ocean island basalt (OIB) type by Wooden et al. [25], whereas the second group of low-Ti rocks were regarded as within-plate basalt (WPB). It was suggested that these two rock groups were produced by the initial rift and subsequent platform stages of magmatic activity within the Siberian trap province [53,58] that changed from one to another over time. Based on their enrichment in LILE, a "transitional series" of intermediate composition comprising Khakanchansky, Tuklonsky Nadezhdinsky, and Lower Morongovsky formations was identified [26,59].

The petrography, geochemistry and mineralogy of volcanic rocks has been presented in numerous publications $[9,14,25,31,34-39,60-62]$. The rock texture changes from aphyric to porphyric and glomeroporphyric. The phenocrysts (3-20 vol. \%) in the latter are represented by plagioclase (90\%), and, in rare cases, by pyroxene or olivine. Groundmass consists of plagioclase and clinopyroxene, sometimes with olivine. The predominant groundmass textures are intersertal, poikilophitic, and tholeiitic. The lowest three formations are characterized by maximal diversity: the Ivakinsky formation consists of trachybasalt and basaltic andesite, the Syverminsky formation contains both tholeiitic and poikilophitic basalt and basaltic andesite, and the Gudchikhinsky Formation comprises basalt and picritic basalt. All overlapped volcanic rocks (the Tuklonsky, Nadezhdinsky, Morongovsky, Mokulaevsky, Kharaelakhsky, Kumginsky, and Samoedsky formations) are very similar texture tholeitic basalt (aphyric or porphyric).

In the Taimyr Peninsula ("2" in Figure 2b) the lower formations (Syradasaysky, Verkhnertamsky, and Labaksky) are OIB-type and similar to those of the initial rift stage in the Noril'sk area. The upper formations at the end of magmatic activity cover the peninsula with WPB (Betlingsky formation). The Ayatarinsky formation is spread throughout eastern Taimyr.

The Maymecha-Kotuy area ("3" in Figure 2b) is well known as an alkaline-ultrabasic province comprising the alkaline Delkansky, Pravoboyarsky, and Arydzhangsky formations and the high-Mg rocks of the Maymechinsky formation. Rocks of normal alkalinity include the Onkuchaksky Formation, while the Tyvankitsky formation contains interlayered normal and subalkaline lavas (Table 1; [31,35,40,54]).

In the Putorana plateau and the central part of the Tunguska syncline (Figure 1a), the WPB-type volcanic rocks constitute the Ayansky and Honnamakitsky formations, which completely correspond to the Morongovsky and Mokulaevsky formations in the Noril'sk region (see captions to Figure 2a). In the southern part of the Tunguska syncline, several formations have been recognized, i.e., Tutonchansky and Korvunchansky (tuffs) and Nidymsky, Kochechumsky and Yambukansky (lavas) [63]. These formations are typical WPB and very close to each other (as will be demonstrated below).

\subsection{Intrusive Rocks}

The intrusions of the Siberian trap province are mostly represented by sill-like bodies inside the sedimentary rocks (S-P) which is below the tuff-lava sequence. On the modern surface they are situated on the periphery of the Siberian trap province (Zone III, Figure 2a). This position does not allow the determination of their sequence of emplacement and relationships with lavas which are important for prospecting of new deposits.

The ore-bearing intrusions have been discovered only in the north part of the province (Figure 1a). They were related to the Noril'sk intrusive complex [64] comprising differentiated ultrabasic-basic bodies. Their characteristics have been given in many publications [8,31,34,45,47,65-70]. The gabbro-dolerite layers were recognized within a vertical cross-section of intrusions (from bottom to top according to the Russian classification): contact, taxitic, picritic, olivine, olivine-bearing, and olivine free. The upper zone of intrusions consists of gabbro-diorites, leucogabbro, and magmatic breccias (with coal xenoliths); and sometimes comprises upper picritic and taxitic gabbro-dolerite.

However, these intrusions have never been compared with intrusions from other areas of the province $[49,50]$, using modern geochemical methods. This has only been done for some intrusive bodies of the Viluy river valley [71]. Below, we briefly describe different intrusions with a focus on their geochemistry in order to reveal the specifics of the Noril'sk ore-bearing intrusions. 


\subsection{PGE-Cu-Ni Deposits}

The sulfide PGE-Cu-Ni Noril'sk deposits are spatially and genetically related to the differentiated intrusions. Three intrusive bodies comprise extra-large deposits, namely the Noril'sk 1, Talnakh, and Kharaelakh (Oktyabr'skoe deposit). Four ore types were recognized: sulfide disseminated ore in the intrusive rocks; sulfide disseminated ore in sedimentary rocks; massive ore in intrusive and surrounding rocks; and low-sulfide ore in the upper zone of the intrusions. The first specific feature of the Noril'sk deposits is a huge thickness of massive orebodies (up to $54 \mathrm{~m}$ ) related to thin (100-150 m) silicate intrusions. Chalcopyrite, pyrrhotite, and pentlandite are the main ore-forming minerals similar to other $\mathrm{Cu}-\mathrm{Ni}$ deposits. Additionally, there are economic ore types consisting of rare minerals of the chalcopyrite group such as talnakhite and moikhukite. The second specific feature of the Noril'sk ore is their enrichment in platinum group elements (5-12 ppm in disseminated ore and up to 125 in massive ore [72]. Due to the high PGE tenor, many PGM have been diagnosed [73-76].

\section{Results}

\subsection{Volcanic Rocks}

The subdivision of volcanic rocks changes from one area to another [35]. Here, we discuss their characteristics based on our data from the key areas (numbers 1-6 in Figure 2b) to identify the specific features of magmatism of the north part of the province.

In the Noril'sk area, the volcanic rocks are well studied and interpreted. Together with OIB and WPB rocks the identification of the "transitional series" was done for two particular sections of volcanic rocks from the west (the Kharaelakh trough) and east parts (the Tunguska syneclise; syneclise is a Russian term which means a huge syncline with long evolution) of the territory [26,59]. They were combined into one section according to the idea that a whole tuff-lava sequence was formed on a flat surface [57], which was folded after the trap magmatism. Indeed, there are a lot of data that demonstrate that the Noril'sk-Kharaelakh trough, Tunguska syneclise, and Khantaysko-Rybninsky swell were formed during Paleozoic times before the trap magmatism event $[8,19]$. Thus, the structure of the volcanic rocks fixes the differences of magmatism development in different tectonic elements.

We analyzed the volcanic sequence including the "transitional series" within the Mikchangdinsky area (Figure 1b) in order to understand the magmatism evolution. I. This area is located at the pericline of the Khantaysko-Rybninsky swell, at the junction of the Noril'sk-Kharaelakh trough and the Tunguska syneclise. A representative sequence of volcanic rocks (560 m thick) in the southern part of the Iken trough was thoroughlystudied in the cross-section (its position of the section is labeled as "Section" in Figure 2b). It comprises five formations: the Syverminsky $\left(\mathrm{T}_{1} \mathrm{sv}\right)$, Gudchikhinsky $\left(\mathrm{T}_{1} \mathrm{gd}\right)$, Khakanchansky, Tuklonsky ( $\left.\mathrm{T}_{1} \mathrm{tk}\right)$, and Nadezhdinsky $\left(\mathrm{T}_{1} \mathrm{nd}\right)$ (Figure 3 ). The last three formations represent so-called "intermediate formations" with intermediate (transitional) geochemical characteristics [26] that differ from those of the formations distributed in the west.

The previous subdivision of rocks into formations inside this section is shown in Figure 3 by black lines. The results of representative analyses are given in Table 2 and are shown in bold and underlined in Figure 3. We have changed the attribution of volcanic rocks to the formations (blue lines, Figure 3), which alternate in the section, and proposed the concept of their origin.

The analytical data (Table 2) confirmed the presence of rocks of the lower formations (Syverminsky and Gudchikhinsky) in the bottom of the section. The Syverminsky formation comprises twelve flows of basalt and trachybasalt with poikilophitic, tholeiitic structure and that are, in total, $110 \mathrm{~m}$ thick. These rocks are overlain by the picritic basalt of the Gudchikhinsky Formation (two flows up to $45 \mathrm{~m}$ in total thickness).

The most important data were obtained for the Khakanchansky and Tuklonsky formations, including lavas and tuffs (Figure 3). This part of the section includes three tuff horizons (3, 12, and $6 \mathrm{~m}$ thick) that are separated by flows of tholeiitic basalt. According to the legend [63] the lowermost layer is traditionally attributed to the Khakanchansky formation, whereas the upper layers belong to 
the Tuklonsky formation. The latter also includes the overlying 12 poikilophitic and 5 tholeiitic flows with a total thickness of $170 \mathrm{~m}$. The Nadezhdinsky formation, which overlaps the Tuklonsky basalt (Figure 3), consists of many flows of porphyritic and aphyric andesitic basalt (Figure 4).

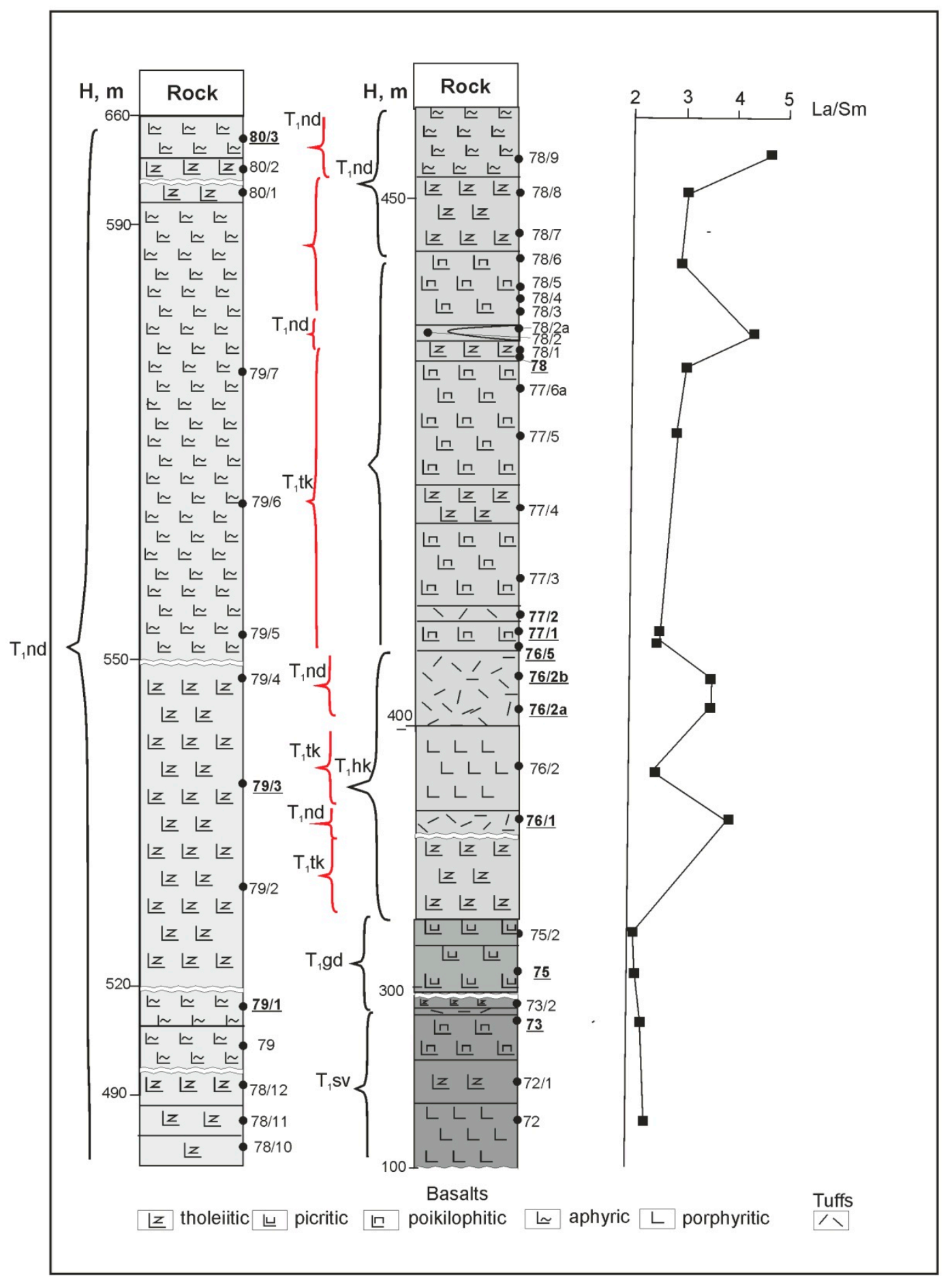

Figure 3. Cross-section of the volcanic rocks in the Mikchangdinsky area.

The volcanic rocks were previously subdivided based on the structures and textures of rocks, whereas the geochemistry of the tuff was ignored. Our geochemical study of this part of the section demonstrates an unexpected result: the compositions of the tuff correspond to the compositions of the Nadezhdinsky rocks and they must be attributed to the Nadezhdinsky formation, whereas the 
compositions of the interlayered basalt are typical of the Tuklonsky formation (red lines in Figure 3). Based on the distribution of the Nadezhdinsky and Tuklonsky volcanic rocks (Figure 5), we concluded that the material was transported from different sources-from the west and east, respectively-to form different rocks at the same place. Thus, based on the Gd/Yb ratio and location within the province [53], the "transitional" rocks were produced by a magmatic process typical of the rift (the Nadezhdinsky formation) and platform (the Tuklonsky formation) regime, rather than by any intermediate process. The difference between the Upper Nadezhdinsky and Lower Morongovsky formations was previously demonstrated $[77,78]$. Thus, trap magmatism per se (the platform stage) began from the eruption of the Tuklonsky basalt, not from the Upper Morongovsky rocks, as was suggested earlier [59]. It began during the magmatic activity of the rift.

An additional evidence of the simultaneous eruption of geochemically different lavas could be provided by the paleomagnetic data. In the Noril'sk volcanic section Heunemann et al. [79] identified the intervals that were formed during the short periods of the anomalous state of the geomagnetic field (the reversal and the following excursion), namely transitional and excursional intervals. As was shown by Pavlov et al. [80] in the Sunduk section (east of the Noril'sk area) the excursional interval comprises the lower part of the Nadezhdinsky formation, while in the Iken section [79] (in the north-east part of the Noril'sk area) it comprises the uppermost Tuklonsky and Lower Nadezhdinsky formations. Since the global geomagnetic events, such as reversals and excursions, are supposed to be isochronous, the data given above, demonstrate that both rift and platform regimes of volcanic activity, were acting simultaneously within this area.

Point and number near the column demonstrate the position of sample in the section and its number. Black lines (brackets) show the previous division of rocks into formations, red lines demonstrate a new version of rocks' subdivision. Position of the cross-section in Figure $2 b$ is labeled as "Section". Data are shown in Table 2.

Table 2. Chemical composition of the volcanic rocks in the Mikchangdinsky area ("Section" in Figure $2 b$ ).

\begin{tabular}{|c|c|c|c|c|c|c|c|c|}
\hline No & 1 & 2 & 3 & 4 & 5 & 6 & 7 & 8 \\
\hline $\begin{array}{c}\mathrm{N} \\
\text { sample }\end{array}$ & 72 & 73 & 75 & $75 / 2$ & $76 / 1$ & $76 / 2$ & $76 / 2 a$ & $76 / 26$ \\
\hline Formation & $\mathrm{sv}$ & $\mathrm{sv}$ & $g_{2}$ & $\mathrm{gd}_{2}$ & hk & $\mathrm{tk}$ & hk & hk \\
\hline Rock & $\mathrm{tb}$ & $\mathrm{b}$ & $\mathrm{pb}$ & $\mathrm{b}$ & $\mathrm{t}$ & $\mathrm{b}$ & $\mathrm{t}$ & $t$ \\
\hline $\mathrm{SiO}_{2}$ & 49.04 & 47.78 & 44.09 & 46.75 & 48.08 & 46.31 & 46.23 & 44.72 \\
\hline $\mathrm{TiO}_{2}$ & 1.23 & 1.41 & 1.24 & 1.66 & 0.85 & 0.87 & 1.02 & 0.81 \\
\hline $\mathrm{Al}_{2} \mathrm{O}_{3}$ & 12.46 & 12.24 & 7.85 & 11.61 & 13.51 & 14.77 & 15.58 & 12.25 \\
\hline $\mathrm{Fe}_{2} \mathrm{O}_{3}$ & 12.15 & 10.36 & 12.89 & 12.7 & 9.08 & 9.9 & 8.73 & 5.37 \\
\hline $\mathrm{MnO}$ & 0.16 & 0.15 & 0.18 & 0.16 & 0.14 & 0.17 & 0.17 & 0.21 \\
\hline $\mathrm{MgO}$ & 8.06 & 8.27 & 17.22 & 10.4 & 9.34 & 9.6 & 7.18 & 3.53 \\
\hline $\mathrm{CaO}$ & 8.95 & 9.6 & 7.74 & 9.73 & 10 & 9.6 & 8.24 & 15.84 \\
\hline $\mathrm{Na}_{2} \mathrm{O}$ & 3.81 & 3.72 & 0.86 & 1.91 & 2.56 & 2.25 & 0.76 & 2.16 \\
\hline $\mathrm{K}_{2} \mathrm{O}$ & 2.42 & 1.19 & 0.11 & 0.45 & 0.45 & 0.77 & 1.48 & 2.33 \\
\hline $\mathrm{P}_{2} \mathrm{O}_{5}$ & 0.14 & 0.13 & 0.111 & 0.13 & 0.09 & 0.087 & 0.122 & 0.175 \\
\hline $\mathrm{Cr}_{2} \mathrm{O}_{3}$ & & 0.083 & 0.188 & & 0.1 & 0.061 & 0.041 & 0.027 \\
\hline LOI & 6.11 & 5.13 & 7.37 & 4.11 & 4.9 & 5.04 & 10.28 & 14.52 \\
\hline Total & 98.42 & 100.06 & 99.84 & 99.61 & 99.1 & 99.43 & 99.83 & 101.94 \\
\hline $\mathrm{Rb}$ & 17.6 & 4.76 & 7.01 & 9.4 & 9.38 & 16.63 & 42.73 & 39 \\
\hline $\mathrm{Sr}$ & 406 & 362 & 147 & 240 & 210 & 272 & 275 & 240 \\
\hline $\mathrm{Y}$ & 23.1 & 20.4 & 14.4 & 18 & 20.3 & 15.2 & 20.7 & 18 \\
\hline $\mathrm{Ba}$ & 206 & 71 & 37 & 126 & 166 & 190 & 291 & 229 \\
\hline $\mathrm{La}$ & 11.9 & 9.87 & 6.41 & 7.9 & 14.15 & 5.86 & 11.57 & 10 \\
\hline $\mathrm{Ce}$ & 26.9 & 22.87 & 15.63 & 20 & 29 & 12.87 & 24.58 & 22 \\
\hline $\operatorname{Pr}$ & 3.76 & 3.15 & 2.19 & 2.9 & 3.54 & 1.68 & 3.25 & 2.9 \\
\hline $\mathrm{Nd}$ & 18.4 & 15 & 10.7 & 13 & 15 & 8 & 15 & 11 \\
\hline
\end{tabular}


Table 2. Cont.

\begin{tabular}{|c|c|c|c|c|c|c|c|c|}
\hline $\mathrm{Sm}$ & 4.84 & 3.87 & 2.78 & 3.8 & 3.41 & 2.22 & 3.6 & 3.1 \\
\hline $\mathrm{Eu}$ & 1.75 & 1.19 & 0.94 & 1.3 & 0.94 & 0.87 & 1.24 & 1.1 \\
\hline $\mathrm{Gd}$ & 4.65 & 4.14 & 3.15 & 3.7 & 3.6 & 2.58 & 3.84 & 2.8 \\
\hline $\mathrm{Tb}$ & 0.77 & 0.65 & 0.48 & 0.66 & 0.58 & 0.43 & 0.6 & 0.53 \\
\hline Dy & 4.67 & 4.16 & 3.05 & 3.9 & 3.87 & 2.94 & 3.97 & 3.3 \\
\hline Ho & 0.91 & 0.81 & 0.57 & 0.77 & 0.78 & 0.6 & 0.8 & 0.71 \\
\hline Er & 2.3 & 2.16 & 1.46 & 2 & 2.24 & 1.68 & 2.25 & 2 \\
\hline $\mathrm{Tm}$ & 0.31 & 0.31 & 0.21 & 0.26 & 0.33 & 0.24 & 0.33 & 0.27 \\
\hline $\mathrm{Yb}$ & 2.03 & 2.01 & 1.31 & 1.6 & 2.17 & 1.65 & 2.18 & 1.8 \\
\hline $\mathrm{Lu}$ & 0.29 & 0.29 & 0.19 & 0.22 & 0.32 & 0.24 & 0.32 & 0.26 \\
\hline $\mathrm{Pb}$ & 2.41 & 1.36 & 2.31 & 1.5 & 5.34 & 2.27 & 4.62 & 2 \\
\hline Th & 1.58 & 1.91 & 0.95 & 0.99 & 3.12 & 0.62 & 2.16 & 1.8 \\
\hline $\mathrm{U}$ & 0.46 & 0.41 & 0.31 & 0.3 & 0.79 & 0.17 & 0.61 & 0.54 \\
\hline Sc & 33.8 & 28.5 & 23.4 & 26 & 33.3 & 37.2 & 36.5 & 29 \\
\hline $\mathrm{Co}$ & 126 & 53 & 111 & 52 & 51 & 56 & 41 & 34 \\
\hline $\mathrm{Ni}$ & 349 & 242 & & 306 & 346 & 153 & 118 & 96 \\
\hline $\mathrm{Cu}$ & 106 & 85 & 73 & 110 & 70 & 82 & 99 & 91 \\
\hline $\mathrm{Zn}$ & 92 & 56 & 118 & & 88 & 85 & 86 & 91 \\
\hline $\mathrm{Zr}$ & 126 & 105 & 81 & 89 & 88 & 55 & 91 & 76 \\
\hline $\mathrm{Nb}$ & 8.94 & 6.05 & 5.71 & 6.6 & 5.67 & 2.65 & 6.34 & 5.8 \\
\hline $\mathrm{Hf}$ & 3.39 & 2.93 & 2.22 & 2.1 & 2.38 & 1.51 & 2.4 & 1.7 \\
\hline $\mathrm{Ta}$ & 0.59 & 0.43 & 0.38 & 0.67 & 0.39 & 0.17 & 0.42 & 0.53 \\
\hline No & 9 & 10 & 11 & 12 & 13 & 14 & 15 & 16 \\
\hline $\begin{array}{c}\mathrm{N} \\
\text { sample }\end{array}$ & $76 / 5$ & $77 / 1$ & $77 / 5$ & 78 & $78 / 2$ & $78 / 2 a$ & $78 / 6$ & $78 / 8$ \\
\hline Formation & tk & tk & tk & tk & $\mathrm{tk}$ & hk & $\mathrm{tk}$ & $\mathrm{nd}_{1}$ \\
\hline Rock & $\mathrm{b}$ & $\mathrm{b}$ & $\mathrm{b}$ & $b$ & ba & $\mathrm{t}$ & $b$ & ba \\
\hline $\mathrm{SiO}_{2}$ & 49.41 & 46.95 & 47.06 & 47.35 & 51.22 & 52.54 & 49.63 & 48.12 \\
\hline $\mathrm{TiO}_{2}$ & 0.89 & 0.79 & 0.92 & 0.93 & 1.02 & 0.83 & 0.95 & 0.91 \\
\hline $\mathrm{Al}_{2} \mathrm{O}_{3}$ & 13.51 & 14.72 & 15.02 & 15.16 & 15.31 & 14.58 & 15.41 & 14.8 \\
\hline $\mathrm{Fe}_{2} \mathrm{O}_{3}$ & 5.96 & 9.62 & 9.75 & 9.96 & 10.35 & 8.13 & 9.86 & 9.42 \\
\hline $\mathrm{MnO}$ & 0.22 & 0.15 & 0.16 & 0.15 & 0.18 & 0.14 & 0.147 & 0.131 \\
\hline $\mathrm{MgO}$ & 6.58 & 9.54 & 8.22 & 8.19 & 8.34 & 4.19 & 7.53 & 6.43 \\
\hline $\mathrm{CaO}$ & 14.63 & 11.02 & 7.6 & 7.2 & 9.73 & 19.13 & 11.87 & 12.34 \\
\hline $\mathrm{Na}_{2} \mathrm{O}$ & 2.63 & 2 & 3.72 & 3.78 & 2.6 & 0.18 & 1.76 & 2.36 \\
\hline $\mathrm{K}_{2} \mathrm{O}$ & 2.91 & 0.21 & 0.66 & 0.63 & 0.66 & 0.02 & 0.12 & 0.14 \\
\hline $\mathrm{P}_{2} \mathrm{O}_{5}$ & 0.2 & 0.083 & 0.096 & 0.098 & 0.15 & 0.09 & 0.097 & 0.1 \\
\hline $\mathrm{Cr}_{2} \mathrm{O}_{3}$ & 0.04 & 0.066 & 0.058 & 0.05 & & & 0.058 & 0.05 \\
\hline LOI & 4.32 & 4.34 & 4.9 & 5.22 & & & 4.46 & 5.54 \\
\hline Total & 101.3 & 99.49 & 98.16 & 98.72 & 99.57 & 99.84 & 101.89 & 100.34 \\
\hline $\mathrm{Rb}$ & 108. & 3.95 & 20.7 & 21.7 & 15.0 & 0.63 & 0.99 & 3.29 \\
\hline $\mathrm{Sr}$ & 456 & 246 & 545 & 394 & 413 & 47 & 277 & 425 \\
\hline $\mathrm{Y}$ & 19.8 & 14.9 & 15.5 & 13.6 & 22.1 & 14.2 & 15.8 & 16.6 \\
\hline $\mathrm{Ba}$ & 494 & 106 & 521 & 290 & 343 & 12 & 142 & 89 \\
\hline $\mathrm{La}$ & 28.31 & 5.12 & 6.22 & 5.74 & 19.67 & 7.43 & 7.07 & 8.25 \\
\hline $\mathrm{Ce}$ & 51.17 & 11.42 & 13.76 & 12.42 & 40.28 & 14.81 & 15.27 & 17.67 \\
\hline $\operatorname{Pr}$ & 5.71 & 1.51 & 1.8 & 1.62 & 4.73 & 1.87 & 2.01 & 2.26 \\
\hline $\mathrm{Nd}$ & 22.7 & 7.3 & 8.5 & 7.6 & 19.7 & 8.6 & 9.1 & 10.3 \\
\hline $\mathrm{Sm}$ & 4.33 & 2.03 & 2.25 & 1.97 & 4.12 & 2.14 & 2.37 & 2.52 \\
\hline $\mathrm{Eu}$ & 1.23 & 0.82 & 0.88 & 0.78 & 1.18 & 0.85 & 0.94 & 0.99 \\
\hline $\mathrm{Gd}$ & 3.99 & 2.49 & 2.57 & 2.33 & 4.09 & 2.49 & 2.81 & 2.92 \\
\hline $\mathrm{Tb}$ & 0.59 & 0.42 & 0.44 & 0.38 & 0.64 & 0.41 & 0.46 & 0.48 \\
\hline Dy & 3.7 & 2.83 & 2.96 & 2.61 & 4.29 & 2.76 & 3.15 & 3.22 \\
\hline Ho & 0.73 & 0.59 & 0.61 & 0.54 & 0.88 & 0.56 & 0.64 & 0.67 \\
\hline Er & 1.94 & 1.67 & 1.69 & 1.48 & 2.4 & 1.55 & 1.71 & 1.87 \\
\hline $\mathrm{Tm}$ & 0.28 & 0.25 & 0.25 & 0.22 & 0.35 & 0.23 & 0.26 & 0.27 \\
\hline $\mathrm{Yb}$ & 1.89 & 1.59 & 1.62 & 1.47 & 2.35 & 1.52 & 1.69 & 1.84 \\
\hline $\mathrm{Lu}$ & 0.27 & 0.24 & 0.24 & 0.22 & 0.36 & 0.23 & 0.26 & 0.27 \\
\hline $\mathrm{Pb}$ & 13.42 & 1.83 & 2.49 & 2.41 & 2.59 & 3.19 & 1.93 & 2.88 \\
\hline Th & 3.5 & 0.66 & 0.71 & 0.66 & 3.07 & 0.69 & 0.8 & 0.85 \\
\hline
\end{tabular}


Table 2. Cont.

\begin{tabular}{|c|c|c|c|c|c|c|c|c|}
\hline $\mathrm{U}$ & 1.09 & 0.17 & 0.17 & 0.15 & 0.73 & 0.19 & 0.33 & 0.23 \\
\hline Sc & 19.5 & 38.5 & 38.1 & 33 & 42.4 & 31.6 & 38.8 & 38.4 \\
\hline Co & 32 & 58 & 51 & 45 & 43 & 37 & 53 & 51 \\
\hline $\mathrm{Ni}$ & 92 & 187 & 115 & 98 & 52 & 65 & 114 & 58 \\
\hline $\mathrm{Cu}$ & 64 & 104 & 77 & 71 & 62 & 16 & 114 & 52 \\
\hline $\mathrm{Zn}$ & 92 & 84 & 89 & 83 & 103 & 50 & 117 & 77 \\
\hline $\mathrm{Zr}$ & 115 & 51 & 56 & 54 & 120 & 54 & 58 & 62 \\
\hline $\mathrm{Nb}$ & 10.36 & 2.59 & 2.78 & 2.67 & 7.51 & 2.82 & 2.76 & 3.1 \\
\hline Hf & 2.81 & 1.39 & 1.5 & 1.41 & 3.09 & 1.43 & 1.5 & 1.68 \\
\hline $\mathrm{Ta}$ & 0.61 & 0.17 & 0.18 & 0.16 & 0.45 & 0.17 & 0.19 & 0.19 \\
\hline No & 17 & 18 & 19 & 20 & 21 & 22 & 23 & 24 \\
\hline $\begin{array}{c}\mathrm{N} \\
\text { sample }\end{array}$ & $78 / 9$ & 79 & $79 / 1$ & $79 / 3$ & $79 / 6$ & $80 / 1$ & BHVO-2 & LOD \\
\hline Formation & $\mathrm{nd}_{1}$ & $\mathrm{nd}_{1}$ & $\mathrm{nd}_{1}$ & $\mathrm{nd}_{2}$ & $\mathrm{nd}_{2}$ & $\mathrm{nd}_{2}$ & & \\
\hline Rock & ba & ba & ba & ba & ba & ba & $\mathrm{b}$ & \\
\hline $\mathrm{SiO}_{2}$ & 51.08 & 51.42 & 51.84 & 49.98 & 51.82 & 50.83 & 49.5 & 0.023 \\
\hline $\mathrm{TiO}_{2}$ & 0.9 & 0.92 & 0.98 & 0.9 & 1 & 1 & 2.71 & 0.007 \\
\hline $\mathrm{Al}_{2} \mathrm{O}_{3}$ & 14.77 & 15 & 14.84 & 14.3 & 14.55 & 14.91 & 13.0 & 0.009 \\
\hline $\mathrm{Fe}_{2} \mathrm{O}_{3}$ & 8.03 & 9.17 & 9.64 & 9.81 & 9.88 & 9.83 & 12.3 & 0.005 \\
\hline $\mathrm{MnO}$ & 0.15 & 0.156 & 0.143 & 0.141 & 0.142 & 0.137 & 0.18 & 0.004 \\
\hline $\mathrm{MgO}$ & 6.01 & 6.6 & 6.84 & 6.2 & 6.72 & 5.81 & 7.23 & 0.007 \\
\hline $\mathrm{CaO}$ & 11.87 & 9.4 & 10.28 & 9.52 & 9.95 & 7.45 & 11.2 & 0.005 \\
\hline $\mathrm{Na}_{2} \mathrm{O}$ & 2.2 & 2.45 & 2.36 & 3.1 & 2.17 & 3.2 & 2.24 & 0.01 \\
\hline $\mathrm{K}_{2} \mathrm{O}$ & 0.88 & 1.2 & 1 & 1.12 & 0.94 & 1.77 & 0.50 & 0.004 \\
\hline $\mathrm{P}_{2} \mathrm{O}_{5}$ & 0.1 & 0.12 & 0.124 & 0.114 & 0.125 & 0.12 & 0.26 & 0.008 \\
\hline $\mathrm{Cr}_{2} \mathrm{O}_{3}$ & 0.04 & 0.04 & 0.023 & 0.059 & 0.036 & 0.023 & & \\
\hline LOI & 3.02 & 2.8 & 1.95 & 5.07 & 2.38 & 3.96 & & \\
\hline Total & 99.05 & 99.27 & 100.02 & 100.31 & 99.71 & 99.04 & & \\
\hline $\mathrm{Rb}$ & 21.7 & 28.0 & 18.7 & 23.7 & 21.3 & 111. & 9.3 & 0.18 \\
\hline $\mathrm{Sr}$ & 251 & 358 & 276 & 375 & 269 & 490 & 376 & 4.15 \\
\hline $\mathrm{Y}$ & 19.7 & 19.1 & 21.7 & 20.6 & 21.8 & 24.7 & 26.7 & 0.05 \\
\hline $\mathrm{Ba}$ & 241 & 352 & 298 & 439 & 294 & 504 & 128 & 1.7 \\
\hline $\mathrm{La}$ & 17.3 & 16.9 & 16.7 & 16.6 & 16.3 & 20.8 & 16.0 & 0.1 \\
\hline $\mathrm{Ce}$ & 34.4 & 33.1 & 35.1 & 34.9 & 34.8 & 41.2 & 40.9 & 0.21 \\
\hline $\operatorname{Pr}$ & 4.22 & 4.04 & 4.35 & 4.26 & 4.35 & 4.86 & 5.5 & 0.02 \\
\hline $\mathrm{Nd}$ & 17.5 & 17 & 18.1 & 17.9 & 18.3 & 20 & 26.4 & 0.1 \\
\hline $\mathrm{Sm}$ & 3.7 & 3.5 & 3.92 & 3.82 & 4.06 & 4.31 & 6.5 & 0.02 \\
\hline $\mathrm{Eu}$ & 1.05 & 1.05 & 1.02 & 1.05 & 1.07 & 1.01 & 2.2 & 0.01 \\
\hline $\mathrm{Gd}$ & 3.65 & 3.6 & 3.98 & 3.85 & 4.17 & 4.48 & 7.2 & 0.03 \\
\hline $\mathrm{Tb}$ & 0.57 & 0.57 & 0.63 & 0.62 & 0.67 & 0.71 & 1.02 & 0.01 \\
\hline Dy & 3.76 & 3.68 & 4.24 & 3.99 & 4.31 & 4.57 & 5.7 & 0.02 \\
\hline Ho & 0.75 & 0.75 & 0.86 & 0.81 & 0.86 & 0.92 & 1.05 & 0.01 \\
\hline Er & 2.12 & 2.11 & 2.39 & 2.28 & 2.39 & 2.66 & 2.8 & 0.01 \\
\hline $\mathrm{Tm}$ & 0.31 & 0.31 & 0.36 & 0.33 & 0.35 & 0.38 & 0.36 & 0.01 \\
\hline $\mathrm{Yb}$ & 2.06 & 2.06 & 2.41 & 2.29 & 2.43 & 2.55 & 2.2 & 0.01 \\
\hline $\mathrm{Lu}$ & 0.31 & 0.32 & 0.36 & 0.33 & 0.36 & 0.39 & 0.31 & 0.01 \\
\hline $\mathrm{Pb}$ & 7.13 & 6.06 & 6.96 & 4.58 & 6.62 & 4.98 & 1.7 & 0.54 \\
\hline Th & 2.78 & 2.43 & 3.53 & 3.13 & 3.57 & 3.53 & 1.4 & 0.04 \\
\hline $\mathrm{U}$ & 0.68 & 0.64 & 1.04 & 0.76 & 0.99 & 0.88 & 0.46 & 0.01 \\
\hline Sc & 32.3 & 35.3 & 34.5 & 37.9 & 34.1 & 34.8 & 31.6 & 0.04 \\
\hline $\mathrm{Co}$ & 39 & 46 & 41 & 46 & 43 & 88 & 43.9 & 0.62 \\
\hline $\mathrm{Ni}$ & 42 & 47 & 22 & 119 & 99 & 146 & 112 & 0.61 \\
\hline $\mathrm{Cu}$ & 53 & 63 & 40 & 136 & 96 & 108 & 125 & 0.14 \\
\hline $\mathrm{Zn}$ & 92 & 118 & 91 & 103 & 121 & 35 & 112 & 1 \\
\hline $\mathrm{Zr}$ & 109 & 105 & 115 & 117 & 114 & 129 & 175 & 0.76 \\
\hline $\mathrm{Nb}$ & 7.86 & 6.71 & 8.64 & 8.09 & 8.23 & 8.32 & 18.7 & 9.5 \\
\hline $\mathrm{Hf}$ & 2.75 & 2.64 & 3.09 & 3.03 & 3 & 3.4 & 4.6 & 0.14 \\
\hline $\mathrm{Ta}$ & 0.45 & 0.41 & 0.57 & 0.47 & 0.5 & 0.52 & 1.1 & 0.22 \\
\hline
\end{tabular}

Note: Here and in Tables 3 and 4 oxides are given in wt \% and elements in ppm. Empty cell-element was not analyzed. Formations: sv-Syverminsky, gd-Gudchikhinsky, hk—Khakanchansky, tk-Tuklonsky, nd-Nadezhdinsky (subformations: nd $_{1}$ - Lower, Middle). Here and in Tables 3 and 4, data for standard sample: BHVO-2-Basalt, Hawaiian Volcanic Observatory, US Geological Survey [30]. LOD means limit of detection. Rocks: 


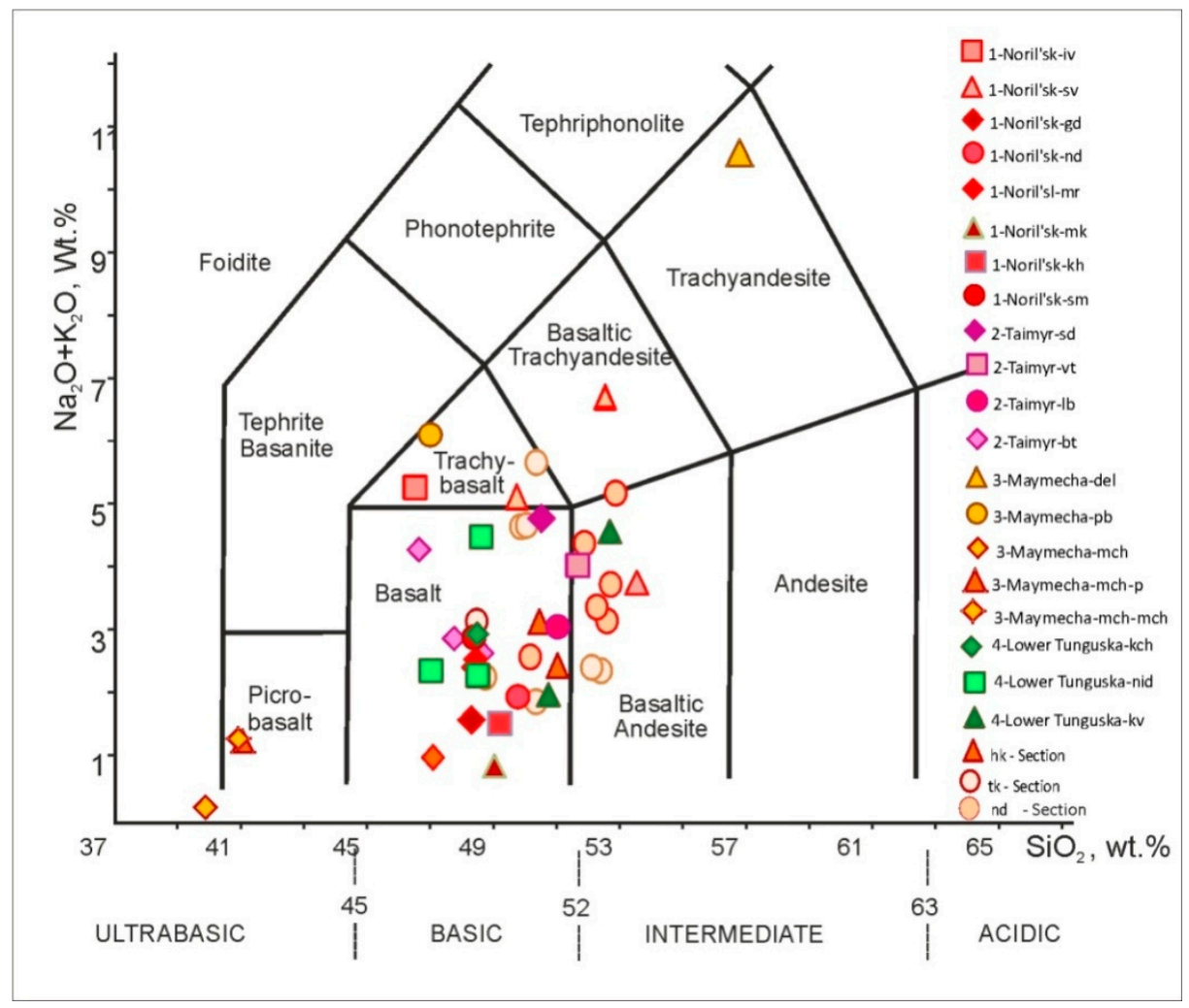

Figure 4. Classification IUGS diagram of $\mathrm{SiO}_{2}-\mathrm{Na}_{2} \mathrm{O}+\mathrm{K}_{2} \mathrm{O}$ for volcanic rocks of the Siberian trap province. Data in Table 3 (analyses in this diagram and in Figures 6, 8 and 9 were calculated to 100\%).
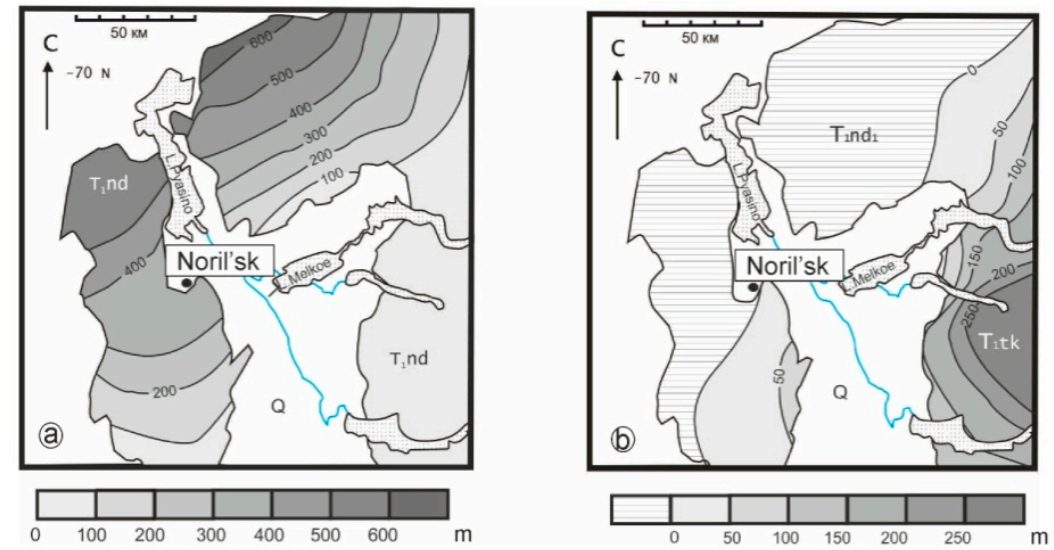

Figure 5. Isopach map for the Nadezhdinsky (a) and Tuklonsky (b) Formations. Data after [14] with changes. Thickness of formations is given in $\mathrm{m}$ (scales under maps).

The Taimyr Peninsula belongs to the Siberian trap province and also includes rift and platform formations. The lowest Syradasaysky formation is an analogue of the Ivakinsky formation [81] in the Noril'sk area. It consists of basalt and subalkaline basalt (Table 3) and is also characterized by reverse magmatic polarity [82]. The Verkhnertamsky (vt) and Labaksky (lb) formations (Table 3, nos. 2 and 3), which overlap the Syradasaysky formation, should correspond to the Syverminsky formation in the Noril'sk area but demonstrate some differences in composition. First, their $\mathrm{TiO}_{2}$ content is lower than those of the Syverminsky formation $(<1 \mathrm{wt} \%$ and $1.4 \mathrm{wt} \%$, respectively). Second, despite the $(\mathrm{Gd} / \mathrm{Yb}) \mathrm{n}$ ratio being higher compared to the platform formations, it only ranges from 1.6 to 1.7, whereas for the Syverminsky trachybasalt it reaches 1.9. Thus, the Verkhnetamsky and Labaksky 
formations are not direct geochemical analogues of the Syverminsky one. The Betlingsky formation (bt, Table 3, no. 4-6) (700 m thick) is located in the west and central Taimyr and consists of tholeiitic and porphyritic basalt with elevated $\mathrm{TiO}_{2}$ contents (average $1.5 \mathrm{wt} \%$ ) and low $(\mathrm{Gd} / \mathrm{Yb}) \mathrm{n}=1.2-1.3$. These data are typical of platform magmatism and allow us to correlate this formation with the Kharaelakhsky formation in the Noril'sk area. This means that the Taimyr Peninsula, similarly to the Noril'sk territory, was covered by WP basalt.

However, the age correlation of the Noril'sk and Taimyr lava sections is not clear. The lowermost Ivakinsky (Noril'sk) and Syradasaysky (Taimyr) formations are likely coeval, because they are geochemically similar, and both are reversely magnetized [79,82] and attributed to the Upper Permian. While all overlying formations in the Noril'sk region have normal magnetic polarity $[79,80]$ (excluding the uppermost Samoedsky formation, for which reliable paleomagnetic data are scarce), the Verkhnetamsky formation of Taimyr reveals a reversed interval [82]. Moreover, there is an opinion that a Middle-Late Triassic magmatic pulse occurred in the Southern Taimyr based on paleomagnetic and ${ }^{40} \mathrm{Ar} /{ }^{39} \mathrm{Ar}$ study of mafic sills and extrusive rocks [83,84]. To resolve the problem, a detailed magnetostratigraphic and geochemical investigation of the Taimyr lava sections is required.

In the Maymecha-Kotuy area, examples of the rocks are the Delkansky (del), Pravoboyarsky (pb), and Maymechinsky (mch) formations shown on Figures 4, 6 and 7c (Table 3). The correlation between the Noril'sk and Maymecha-Kotuy sequences is still being debated. Fedorenko and Czamanske [40] and Arndt and co-authors [41] placed the latter over the former and estimated the entire thickness of volcanic rocks as $6.5 \mathrm{~km}$. Indeed, the rocks of the Noril'sk formations are absent in the Maymecha-Kotuy area, and are replaced by alkaline rocks along the northern boundary of the Siberian platform. Thus, these two sequences do not form one section.

Table 3. Chemical composition of the volcanic rocks from the Siberian trap province.

\begin{tabular}{|c|c|c|c|c|c|c|c|c|}
\hline No & 1 & 2 & 3 & 4 & 5 & 6 & 7 & 8 \\
\hline $\begin{array}{c}\mathrm{N} \\
\text { sample }\end{array}$ & ps3 & $102 \_5$ & 102_1 & LP-5/130 & LP-5/141 & LP-5/145 & 611 & P-28 \\
\hline Formation & $\mathrm{sd}$ & vt & $\mathrm{lb}$ & bt & bt & bt & del & $\mathrm{pb}$ \\
\hline Rock & $\mathrm{b}$ & $\mathrm{b}$ & $\mathrm{b}$ & $\mathrm{b}$ & $\mathrm{b}$ & $\mathrm{b}$ & ta & $\mathrm{tb}$ \\
\hline $\mathrm{SiO}_{2}$ & 50.1 & 49.3 & 51.3 & 42.95 & 45.61 & 45.43 & 55.53 & 45.2 \\
\hline $\mathrm{TiO}_{2}$ & 2.77 & 0.97 & 0.96 & 1.52 & 1.48 & 1.33 & 1.15 & 3.59 \\
\hline $\mathrm{Al}_{2} \mathrm{O}_{3}$ & 14.4 & 13.9 & 15.5 & 12.76 & 14.68 & 11.78 & 18.09 & 10.13 \\
\hline $\mathrm{Fe}_{2} \mathrm{O}_{3}$ & 14.1 & 10.2 & 10.5 & 17.35 & 12.74 & 14.98 & 6.63 & 12.32 \\
\hline $\mathrm{MnO}$ & 0.15 & 0.16 & 0.16 & 0.173 & 0.121 & 0.163 & 0.14 & 0.3 \\
\hline $\mathrm{MgO}$ & 3.44 & 6.4 & 7.06 & 5.97 & 7.13 & 7.7 & 2.02 & 6.98 \\
\hline $\mathrm{CaO}$ & 7.6 & 9.43 & 10.7 & 6.11 & 9.64 & 8.26 & 2.45 & 10.14 \\
\hline $\mathrm{Na}_{2} \mathrm{O}$ & 2.83 & 2.55 & 2.33 & 3.8 & 2.6 & 2.11 & 4.97 & 4.12 \\
\hline $\mathrm{K}_{2} \mathrm{O}$ & 1.88 & 1.29 & 0.75 & 0.12 & 0.14 & 0.36 & 5.27 & 1.7 \\
\hline $\mathrm{P}_{2} \mathrm{O}_{5}$ & 0.74 & 0.12 & 0.1 & 0.14 & 0.16 & 0.14 & 0.5 & 0.48 \\
\hline LOI & 1.76 & 5.71 & 0.93 & 8.79 & 5.48 & 7.5 & 2.64 & 4.29 \\
\hline Total & 99.77 & 100.03 & 100.29 & 99.68 & 99.78 & 99.75 & 99.39 & 99.25 \\
\hline $\mathrm{Rb}$ & 20.2 & 34.3 & 6.78 & 4.2 & 0.6 & 5.8 & 63.8 & 49 \\
\hline $\mathrm{Sr}$ & 376 & 228 & 262 & 187 & 238 & 234 & 856 & 1840 \\
\hline Y & 37.9 & 20.2 & 20.2 & 22.8 & 23.2 & 26 & 32.9 & 23.4 \\
\hline $\mathrm{Ba}$ & 814 & 245 & 241 & 95 & 120 & 168 & 1649 & 2079 \\
\hline $\mathrm{La}$ & 42.7 & 17.3 & 15.3 & 6.9 & 7.26 & 7.7 & 94 & 166 \\
\hline $\mathrm{Ce}$ & 92.5 & 36.3 & 32.1 & 17.5 & 16.9 & 18.5 & 229 & 322 \\
\hline Pr & 11.0 & 4.17 & 3.81 & 2.51 & 2.47 & 2.59 & 27.3 & 33.99 \\
\hline $\mathrm{Nd}$ & 45.7 & 16.8 & 15.8 & 12.2 & 11.4 & 12.7 & 110 & 122 \\
\hline $\mathrm{Sm}$ & 9.7 & 3.81 & 3.64 & 3.41 & 3.37 & 3.86 & 17.8 & 15.8 \\
\hline $\mathrm{Eu}$ & 3.22 & 1.13 & 1.11 & 1.09 & 1.22 & 1.37 & 4.58 & 3.95 \\
\hline $\mathrm{Gd}$ & 10.2 & 4.17 & 4 & 3.66 & 3.83 & 4.19 & 13.5 & 10.33 \\
\hline $\mathrm{Tb}$ & 1.45 & 0.66 & 0.64 & 0.71 & 0.71 & 0.79 & 1.66 & 1.24 \\
\hline
\end{tabular}


Table 3. Cont

\begin{tabular}{|c|c|c|c|c|c|c|c|c|c|}
\hline Dy & 7.64 & 3.82 & 3.78 & 4.43 & 4.55 & 5.05 & 8.99 & 5.98 & \\
\hline Ho & 1.49 & 0.83 & 0.79 & 0.99 & 0.97 & 1.11 & 1.53 & 0.98 & \\
\hline $\mathrm{Er}$ & 4.11 & 2.27 & 2.22 & 2.69 & 2.7 & 2.99 & 4.11 & 2.43 & \\
\hline $\mathrm{Tm}$ & 0.55 & 0.31 & 0.31 & 0.42 & 0.42 & 0.44 & 0.53 & 0.29 & \\
\hline $\mathrm{Yb}$ & 3.36 & 2.08 & 2.00 & 2.57 & 2.46 & 2.66 & 3.36 & 1.89 & \\
\hline $\mathrm{Lu}$ & 0.49 & 0.30 & 0.29 & 0.38 & 0.37 & 0.4 & 0.46 & 0.27 & \\
\hline $\mathrm{Pb}$ & 7.09 & 5.43 & 7.34 & 5.25 & 2.13 & 2.28 & 16.24 & 7.23 & \\
\hline Th & 3.96 & 2.87 & 2.49 & 1.31 & 0.97 & 1.14 & 12.4 & 16.7 & \\
\hline $\mathrm{U}$ & 1.05 & 0.90 & 0.77 & 0.41 & 0.28 & 0.38 & 2.10 & 2.85 & \\
\hline Sc & 21.6 & 29.8 & 30.8 & 38.8 & 38 & 37.1 & 6.0 & 24.2 & \\
\hline $\mathrm{V}$ & 142 & 217 & 220 & 321 & 289 & 303 & 25.0 & 57 & \\
\hline $\mathrm{Cr}$ & 41 & 96 & 124 & 129 & 138 & 123 & 5.00 & & \\
\hline $\mathrm{Co}$ & 31 & 40 & 40 & 53.9 & 42.4 & 48.4 & 6.60 & 55.2 & \\
\hline $\mathrm{Ni}$ & 34 & 15 & 20 & 105 & 92.5 & 81.9 & 3.80 & 79.3 & \\
\hline $\mathrm{Cu}$ & 25 & 19 & 127 & 227 & 126 & 185 & 9.00 & 388 & \\
\hline $\mathrm{Zn}$ & 116 & 73 & 108 & 188 & 67 & 106 & 159 & 138 & \\
\hline $\mathrm{Zr}$ & 278 & 122 & 108 & 111 & 88 & 102 & 415 & 422 & \\
\hline $\mathrm{Nb}$ & 19.4 & 6.35 & 5.4 & 4.67 & 3.56 & 4.75 & 121 & 159 & \\
\hline Hf & 5.56 & 2.33 & 2.02 & 2.4 & 1.2 & 2.64 & 11.7 & 10.2 & \\
\hline $\mathrm{Ta}$ & 1.20 & 0.43 & 0.36 & 0.31 & 0.24 & 0.31 & 6.97 & 10.0 & \\
\hline $\mathrm{La} / \mathrm{Sm}$ & 4.41 & 4.53 & 4.20 & 2.03 & 2.15 & 2.00 & 5.29 & 10.5 & \\
\hline $\mathrm{Gd} / \mathrm{Yb}$ & 3.03 & 2.00 & 2.00 & 1.43 & 1.56 & 1.58 & 4.01 & 5.47 & \\
\hline No & 9 & 10 & 11 & 12 & 13 & 14 & 15 & 16 & 17 \\
\hline $\begin{array}{c}\mathrm{N} \\
\text { sample }\end{array}$ & 160 & 142 & 242 & 290 & 27 & 318 & 4270 & $4270 / 8$ & xc51/99.7 \\
\hline Formation & $\mathrm{kch}$ & nid & nid & nid & $\mathrm{kv}$ & $\mathrm{kv}$ & iv & SV & $\mathrm{gd}$ \\
\hline Rock & $b$ & $b$ & $b$ & $b$ & $b$ & $b$ & $\mathrm{tb}$ & ba & $\mathrm{pb}$ \\
\hline $\mathrm{SiO}_{2}$ & 46.67 & 46.94 & 45.53 & 46.31 & 46.23 & 49.75 & 45.14 & 53.02 & 47.47 \\
\hline $\mathrm{TiO}_{2}$ & 1.6 & 1.32 & 1.49 & 1.34 & 1.37 & 1.02 & 3.91 & 1.85 & 1.01 \\
\hline $\mathrm{Al}_{2} \mathrm{O}_{3}$ & 14.11 & 14.26 & 16.2 & 14.97 & 10.6 & 13.95 & 13.46 & 14.44 & 7.96 \\
\hline $\mathrm{Fe}_{2} \mathrm{O}_{3}$ & 13.71 & 13.55 & 12.5 & 12.08 & 13.54 & 10.29 & 14.55 & 10.43 & 13.36 \\
\hline $\mathrm{MnO}$ & 0.192 & 0.313 & 0.194 & 0.179 & 0.2 & 0.17 & 0.205 & 0.153 & 0.16 \\
\hline $\mathrm{MgO}$ & 5.82 & 6.66 & 4.35 & 6.32 & 6.21 & 4.26 & 3.58 & 5.10 & 20.57 \\
\hline $\mathrm{CaO}$ & 9.96 & 7.91 & 11.9 & 10.78 & 9.89 & 6.62 & 10.54 & 8.95 & 5.35 \\
\hline $\mathrm{Na}_{2} \mathrm{O}$ & 2.4 & 3.8 & 2.15 & 2.08 & 1.41 & 3.51 & 3.35 & 2.54 & 1.21 \\
\hline $\mathrm{K}_{2} \mathrm{O}$ & 0.43 & 0.51 & 0.15 & 0.12 & 0.41 & 0.65 & 1.95 & 1.17 & 0.37 \\
\hline $\mathrm{P}_{2} \mathrm{O}_{5}$ & 0.17 & 0.13 & 0.15 & 0.13 & 0.16 & 0.12 & 0.12 & 0.26 & 0.11 \\
\hline LOI & 4.7 & 3.66 & 5.12 & 5.49 & 9.69 & 6.39 & 3.87 & 2.98 & 3.81 \\
\hline Total & 99.76 & 99.05 & 99.73 & 99.80 & 99.71 & 96.73 & 100.68 & 100.89 & 100.86 \\
\hline $\mathrm{Rb}$ & 8.5 & 12.9 & 2.8 & 1.4 & 10.1 & 20.1 & 30.7 & 40.9 & 3.1 \\
\hline $\mathrm{Sr}$ & 184 & 278 & 223 & 218 & 36 & 366 & 503 & 367 & 108 \\
\hline Y & 27.8 & 25.9 & 26.3 & 24.8 & 27.7 & 24.1 & 46.9 & 32.2 & 15.2 \\
\hline $\mathrm{Ba}$ & 130 & 214 & 52 & 59 & 94 & 261 & 986 & 377 & 33 \\
\hline $\mathrm{La}$ & 9.17 & 8.48 & 7.95 & 6.84 & 9.71 & 13.39 & 43.2 & 25.4 & 7.34 \\
\hline $\mathrm{Ce}$ & 21.2 & 18.2 & 19 & 16.4 & 22 & 30.8 & 102 & 55.9 & 17.8 \\
\hline $\operatorname{Pr}$ & 3.01 & 2.53 & 2.7 & 2.35 & 2.97 & 3.62 & 13.02 & 7.01 & 2.48 \\
\hline $\mathrm{Nd}$ & 14.4 & 12.1 & 12.6 & 11.9 & 14 & 16 & 52.6 & 28.6 & 11.8 \\
\hline Sm & 4.08 & 3.52 & 3.65 & 3.44 & 4 & 3.83 & 10.5 & 6.24 & 3.05 \\
\hline $\mathrm{Eu}$ & 1.36 & 1.33 & 1.24 & 1.14 & 1.13 & 1.17 & 2.80 & 1.82 & 0.90 \\
\hline $\mathrm{Gd}$ & 4.53 & 4.02 & 4.29 & 3.7 & 4.87 & 4.45 & 9.59 & 6.08 & 3.30 \\
\hline $\mathrm{Tb}$ & 0.81 & 0.72 & 0.77 & 0.70 & 0.81 & 0.70 & 1.50 & 0.97 & 0.51 \\
\hline Dy & 5.19 & 4.82 & 5.07 & 4.76 & 5.30 & 4.37 & 8.82 & 5.68 & 3.15 \\
\hline Ho & 1.13 & 1.04 & 1.08 & 1.03 & 1.08 & 0.91 & 1.80 & 1.14 & 0.59 \\
\hline $\mathrm{Er}$ & 3.06 & 2.89 & 2.94 & 2.77 & 3.22 & 2.74 & 4.76 & 3.02 & 1.57 \\
\hline $\mathrm{Tm}$ & 0.44 & 0.41 & 0.43 & 0.41 & 0.45 & 0.38 & 0.70 & 0.44 & 0.21 \\
\hline $\mathrm{Yb}$ & 2.82 & 2.60 & 2.87 & 2.65 & 3.09 & 2.67 & 4.23 & 2.72 & 1.37 \\
\hline $\mathrm{Lu}$ & 0.43 & 0.38 & 0.44 & 0.40 & 0.46 & 0.40 & 0.63 & 0.38 & 0.19 \\
\hline $\mathrm{Pb}$ & 1.53 & 8.95 & 2.23 & 0.08 & 2.95 & 4.19 & 8.98 & 7.30 & 1.81 \\
\hline Th & 1.03 & 0.9 & 0.81 & 0.74 & 1.55 & 2.91 & 3.42 & 4.48 & 1.15 \\
\hline $\mathrm{U}$ & 0.38 & 0.53 & 0.33 & 0.30 & 0.64 & 0.92 & 2.07 & 1.16 & 0.29 \\
\hline Sc & 36.6 & 38 & 39.6 & 36.6 & 45.3 & 28.7 & 18.9 & 25.7 & \\
\hline
\end{tabular}


Table 3. Cont.

\begin{tabular}{|c|c|c|c|c|c|c|c|c|c|c|}
\hline $\mathrm{V}$ & 308 & 290 & 319 & 290 & 338 & 211 & 175 & 189 & & \\
\hline $\mathrm{Cr}$ & 113 & 170 & 179 & 165 & 171 & 110 & 49 & 322 & & \\
\hline $\mathrm{Co}$ & 48.4 & 66.4 & 52.3 & 49.5 & 56.1 & 40 & 34.2 & 35.8 & 118.0 & \\
\hline $\mathrm{Ni}$ & 80 & 120 & 126 & 121 & 117 & 84.2 & 25.9 & 70.5 & 1148 & \\
\hline $\mathrm{Cu}$ & 179 & 386 & 210 & 156 & 134 & 37 & 43.4 & 38.5 & 89.9 & \\
\hline $\mathrm{Zn}$ & 168 & 326 & 259 & 115 & 110 & 81 & 183 & 98 & & \\
\hline $\mathrm{Zr}$ & 110 & 95 & 103 & 88 & 100 & 100 & 399 & 221 & 93 & \\
\hline $\mathrm{Nb}$ & 5.97 & 4.37 & 5.54 & 3.39 & 5.18 & 5.63 & 40.30 & 17.96 & 6.65 & \\
\hline Hf & 3.11 & 2.42 & 2.62 & 2.19 & 2.77 & 2.68 & 8.45 & 5.35 & 2.38 & \\
\hline $\mathrm{Ta}$ & 0.35 & 0.3 & 2.53 & 0.57 & 0.29 & 0.35 & 2.51 & 1.06 & 0.43 & \\
\hline $\mathrm{La} / \mathrm{Sm}$ & 2.24 & 2.41 & 2.18 & 1.99 & 2.43 & 3.5 & 3.5 & 3.5 & 3.5 & \\
\hline $\mathrm{Gd} / \mathrm{Yb}$ & 1.61 & 1.55 & 1.50 & 1.40 & 1.58 & 1.67 & 1.67 & 1.67 & 1.67 & \\
\hline No & 18 & 19 & 20 & 21 & 22 & 23 & 24 & 25 & 24 & 25 \\
\hline$\underset{\text { sample }}{\mathrm{N}}$ & CY-45 & $126 / 8$ & $4002 / 5$ & $4002 / 18$ & $\begin{array}{c}\mathrm{s} \\
16 \mathrm{~F}-13 \\
245^{*}\end{array}$ & $\underset{* *}{3008-10}$ & $\underset{* *}{2 \mathrm{FG}-102}$ & $\underset{* *}{2 \mathrm{FR}}-45$ & BHVO-2 & LOD \\
\hline Formation & nd & $\mathrm{mr}$ & $\mathrm{mk}$ & $\mathrm{kh}$ & $\mathrm{sm}$ & mch & mch & mch & basalt & \\
\hline Rock & $\mathrm{b}$ & $\mathrm{b}$ & $b$ & $\mathrm{~b}$ & $\mathrm{~b}$ & mch & $\mathrm{p}$ & mch & $\mathrm{b}$ & \\
\hline $\mathrm{SiO}_{2}$ & 49.98 & 48.12 & 49.2 & 49.72 & 48.9 & 40.49 & 40.95 & 40.65 & 49.70 & 0.04 \\
\hline $\mathrm{TiO}_{2}$ & 0.76 & 1.04 & 1.25 & 1.29 & 1.57 & 1.91 & 3.31 & 3.82 & 2.72 & 0.005 \\
\hline $\mathrm{Al}_{2} \mathrm{O}_{3}$ & 16.20 & 15.56 & 15.83 & 15.82 & 15.2 & 2.05 & 3.43 & 3.7 & 13.62 & 0.02 \\
\hline $\mathrm{Fe}_{2} \mathrm{O}_{3}$ & 10.64 & 12.60 & 12.72 & 12.93 & 13.76 & 14.22 & 14.63 & 14.75 & 12.30 & 0.005 \\
\hline $\mathrm{MnO}$ & 0.03 & 0.24 & 0.21 & 0.21 & 0.21 & 0.2 & 0.22 & 0.22 & 0.16 & 0.002 \\
\hline $\mathrm{MgO}$ & 7.48 & 6.07 & 6.56 & 6.98 & 6.8 & 37.22 & 27.13 & 25.24 & 7.25 & 0.007 \\
\hline $\mathrm{CaO}$ & 12.06 & 11.76 & 12.36 & 11.21 & 10.3 & 4.12 & 8.52 & 9.78 & 11.30 & 0.003 \\
\hline $\mathrm{Na}_{2} \mathrm{O}$ & 1.72 & 1.55 & 0.80 & 1.38 & 2.44 & 0.03 & 0.31 & 0.34 & 2.19 & 0.01 \\
\hline $\mathrm{K}_{2} \mathrm{O}$ & 0.26 & 0.98 & 0.09 & 0.19 & 0.49 & 0.06 & 1 & 1.01 & 0.52 & 0.004 \\
\hline $\mathrm{P}_{2} \mathrm{O}_{5}$ & 0.09 & 0.19 & 0.12 & 0.13 & 0.17 & 0.24 & 0.5 & 0.54 & 0.26 & 0.008 \\
\hline LOI & 1.21 & 2.34 & 1.61 & 1.10 & 1.70 & & 3.91 & 6.78 & & \\
\hline Total & 100.44 & 100.44 & 100.73 & 100.94 & 101.54 & 100.54 & 100 & 100.05 & & \\
\hline $\mathrm{Rb}$ & 3.5 & 22.0 & 1.96 & 11.73 & 10.6 & 2.55 & 33 & 30 & 9.24 & 0.18 \\
\hline $\mathrm{Sr}$ & 218 & 279 & 210 & 239 & 187 & 277 & 450 & 580 & 389 & 4.15 \\
\hline Y & 15.6 & 30.2 & 23.8 & 28.1 & 27.6 & 9.40 & 16 & 16 & 25.7 & 0.05 \\
\hline $\mathrm{Ba}$ & 105 & 317 & 108 & 120 & 153 & 163 & 450 & 350 & 131 & 1.7 \\
\hline $\mathrm{La}$ & 5.35 & 15.3 & 6.39 & 9.98 & 8.26 & 26.0 & 50.6 & 60 & 15.2 & 0.1 \\
\hline $\mathrm{Ce}$ & 12.2 & 32.7 & 15.4 & 22.2 & 20.4 & 54.8 & 103.2 & 120 & 37.4 & 0.21 \\
\hline $\operatorname{Pr}$ & 1.66 & 4.03 & 2.10 & 2.84 & 2.89 & 7.04 & & & 5.34 & 0.02 \\
\hline $\mathrm{Nd}$ & 7.8 & 16.6 & 9.8 & 12.5 & 13.5 & 30.3 & 48.5 & 58.4 & 24 & 0.1 \\
\hline Sm & 2.22 & 3.70 & 2.74 & 3.28 & 3.69 & 5.70 & 9.5 & 10.8 & 6.06 & 0.02 \\
\hline $\mathrm{Eu}$ & 0.91 & 1.10 & 0.93 & 1.07 & 1.4 & 1.55 & 2.42 & 2.89 & 2.04 & 0.01 \\
\hline $\mathrm{Gd}$ & 2.65 & 4.11 & 3.14 & 3.74 & 4.64 & 3.97 & & 7.59 & 5.9 & 0.03 \\
\hline $\mathrm{Tb}$ & 0.44 & 0.70 & 0.54 & 0.64 & 0.82 & 0.48 & 0.75 & 0.93 & 0.92 & 0.01 \\
\hline Dy & 2.99 & 4.73 & 3.54 & 4.22 & 5.38 & 2.50 & & & 5.3 & 0.02 \\
\hline Ho & 0.61 & 1.07 & 0.82 & 0.95 & 1.06 & 0.38 & & & 0.99 & 0.01 \\
\hline $\mathrm{Er}$ & 1.62 & 2.98 & 2.16 & 2.54 & 2.97 & 0.91 & & & 2.5 & 0.01 \\
\hline $\mathrm{Tm}$ & 0.23 & 0.45 & 0.32 & 0.37 & 0.45 & 0.117 & & & 0.31 & 0.01 \\
\hline $\mathrm{Yb}$ & 1.61 & 2.91 & 1.99 & 2.39 & 2.86 & 0.63 & 0.92 & 1.17 & 1.97 & 0.01 \\
\hline $\mathrm{Lu}$ & 0.23 & 0.44 & 0.30 & 0.36 & 0.43 & 0.08 & 0.13 & 0.143 & 0.27 & 0.01 \\
\hline $\mathrm{Pb}$ & 0.72 & 3.97 & 2.03 & 3.07 & & 0.08 & & & 1.52 & 0.54 \\
\hline Th & 0.75 & 2.58 & 0.94 & 1.20 & 0.98 & 2.50 & 4.45 & 5.4 & 1.29 & 0.04 \\
\hline $\mathrm{U}$ & 0.18 & 1.14 & 0.37 & 0.67 & 0.38 & 0.48 & 1.02 & 1.20 & 0.42 & 0.01 \\
\hline Sc & & 33.9 & 35.9 & 40.9 & & 16.5 & 23 & 24 & 31.5 & 0.04 \\
\hline V & & 211 & 273 & 274 & & 64 & 260 & 140 & 16341 & 1.32 \\
\hline $\mathrm{Cr}$ & & 75 & 101 & 109 & & & 1300 & 1800 & 319 & 0.21 \\
\hline $\mathrm{Co}$ & 40.5 & 40.8 & 47.6 & 52.5 & 43 & 153 & 114 & 101 & 288 & 0.62 \\
\hline $\mathrm{Ni}$ & 144 & 75.3 & 77.7 & 110 & 101 & 2310 & 1100 & 1000 & 1350 & 0.61 \\
\hline $\mathrm{Cu}$ & 112 & 44.3 & 89.2 & 112 & 186 & 67.2 & 100 & 126 & 44.8 & 0.14 \\
\hline $\mathrm{Zn}$ & & 86 & 76 & 101 & & 64.6 & 88 & 108 & 120 & 1 \\
\hline $\mathrm{Zr}$ & 53 & 117 & 80 & 94 & 115 & 179 & 255 & 305 & 131 & 0.76 \\
\hline $\mathrm{Nb}$ & 2.99 & 8.59 & 4.23 & 3.11 & 5.07 & 31.8 & & & 97.3 & 9.5 \\
\hline
\end{tabular}


Table 3. Cont.

\begin{tabular}{ccccccccccc}
\hline $\mathrm{Hf}$ & 1.37 & 2.85 & 1.95 & 2.38 & 2.93 & 3.91 & 5.83 & 6.62 & 172 & 0.14 \\
$\mathrm{Ta}$ & 0.20 & 0.47 & 0.23 & 0.13 & 0.3 & 2.05 & 3.78 & 4.50 & 17.9 & 0.22 \\
$\mathrm{La} / \mathrm{Sm}$ & 3.5 & 3.5 & 3.5 & 3.5 & 3.5 & 4.56 & 5.33 & 5.56 & 2.51 & 5.00 \\
$\mathrm{Gd} / \mathrm{Yb}$ & 1.67 & 1.67 & 1.67 & 1.67 & 1.67 & 6.30 & & 6.49 & 2.99 & 3.00 \\
\hline
\end{tabular}

Note: No. 1-6, Taimyr area; 7-9, Maymecha-Kotuy area; 10-14, Lower Tunguska river valley; 15-22, Noril'sk area, formations: sd—Syradasaysky, vt—Verkhentamsky, lb—Labaksky, bt—Betlingsky, del—Delkansky, kch—Kochechumsky, nid-Nidymsky, kv—Korvunchansky, iv—Ivakinsky, sv—Syverminsky, gd—Gudchikhinsky, nd-Nadezhdinsky, mr-Morongovsky, mk—Mokulaevsky, hr-Kharaelakhsky, sm—Samoedsky. Data: * after [81], ** after [40].

The more realistic positions of these rocks were obtained using paleomagnetic data. It was shown that the Arydzhangsky (ar) and the Lower Onkuchaksky (onk) formations are normally magnetized, whereas the Upper Onkuchaksky formation [54] and the overlying Tyvankitsky (tv) and Lower Delkansky formations [85] have reversed polarity. Based on the comparison of the virtual geomagnetic poles of the volcanic units from the Noril'sk and Kotuy sections, some authors [54] proposed that the Arydzhangsky and lowermost Onkuchaksky formations correlate with the Morongovsky-Mokulaevsky level of the Noril'sk volcanic sequence. According to this scheme, the reversely magnetized uppermost Onkuchaksky and Tyvankitsky as well as the overlying formations of the Maymecha-Kotuy regions should be younger than the Noril'sk section.

Indeed, only the basalts and picritic basalts of the Gudchikhinsky formation, which are distributed in the north of the Noril'sk region [86], are typical mantle rocks (OIB-type), whereas the remaining rocks have typical crustal geochemical characteristics (Tables 2 and 3; Figure 7a). Mantle rocks are also found in the Maymecha-Kotuy region $[40,41,87,88]$. They include the maymechite (or meimechite, maimechite) of the Maymechinsky formation and the alkaline-ultramafic rocks of the Delkansky (trachyandeste), Pravoboyarsky (Figure 7c) and Arydzhangsky formations. All are localized along the Yenisei-Khatanga fault (YKhf in Figure 1b), which provides access to mantle magmas (based on geochemistry of rocks without $\mathrm{Ta}-\mathrm{Nb}$ and $\mathrm{Pb}$ anomalies in their patterns and $\varepsilon_{\mathrm{Nd}} \geq+3$ ) and was active during the entire period of trap emplacement: the Gudchikhinsky magmas intruded along it at an earlier stage and alkaline-ultramafic magmas intruded during the middle stage (Arydzhangsky formation) and the end (Delkansky and Maymechinsky formations) of magmatic evolution (according to their position in cross-section and paleomagnetic data [54]).

In the Tunguska syneclise, the Nidymsky (nid) and Kochechumsky ( $\mathrm{kch}$ ) formations have been studied in the south part of the Tunguska syneclise (the Lower Tunguska river valley). They are very close in composition and correspond in terms of $\mathrm{TiO}_{2}$ content $(1.0-1.6 \mathrm{wt} \%)$ to the upper formations of the Noril'sk area, the Mokulaevsky, and Kharaelakhsky (Figure 6c). The widely distributed tuff of the Korvunchansky formation $(\mathrm{kv})$, which comprise extensive fields to the south of the lava distribution area, have similar geochemical characteristics to the basalt of the main trap stage located in the south of the Tunguska syneclise (Nidymsky and Kochechumsky Formations).

As was shown by Latyshev et al. [89], the major part of the volcanic sequence of the Tunguska syneclise (from the uppermost Korvunchansky up to Kochechumsky formations) comprises the single interval of the normal magnetic polarity. This fact confirms the correlation of the Lower Tunguska lava section (from the uppermost Korvunchansky up to Kochechumsky formations) to the upper part of the Noril'sk volcanic sequence.

All analysis of the effusive rocks represented in Tables 2 and 3 (and numerous published data on the compositions of Siberian traps summarized in the GEOROCK database [90]) indicate that their maximal diversity is typical of the Noril'sk and Maymecha-Kotuy areas, where the high-Mg rocks (Gudchikhinsky and Maymechinsky formations: picritic basalt, picrite, and maymecite) with MgO contents of up to $40 \mathrm{wt} \%$ were revealed. The compositional points of the rocks from the central part of the Tunguska syneclise are located near a field of points of tholeiitic basalts that constitute the upper 
part of the Norilsk sequence with $\mathrm{MgO}$ content of 6-7 wt \% (for clarity, they are shown in the enlarged part of the diagram in Figure 6b).
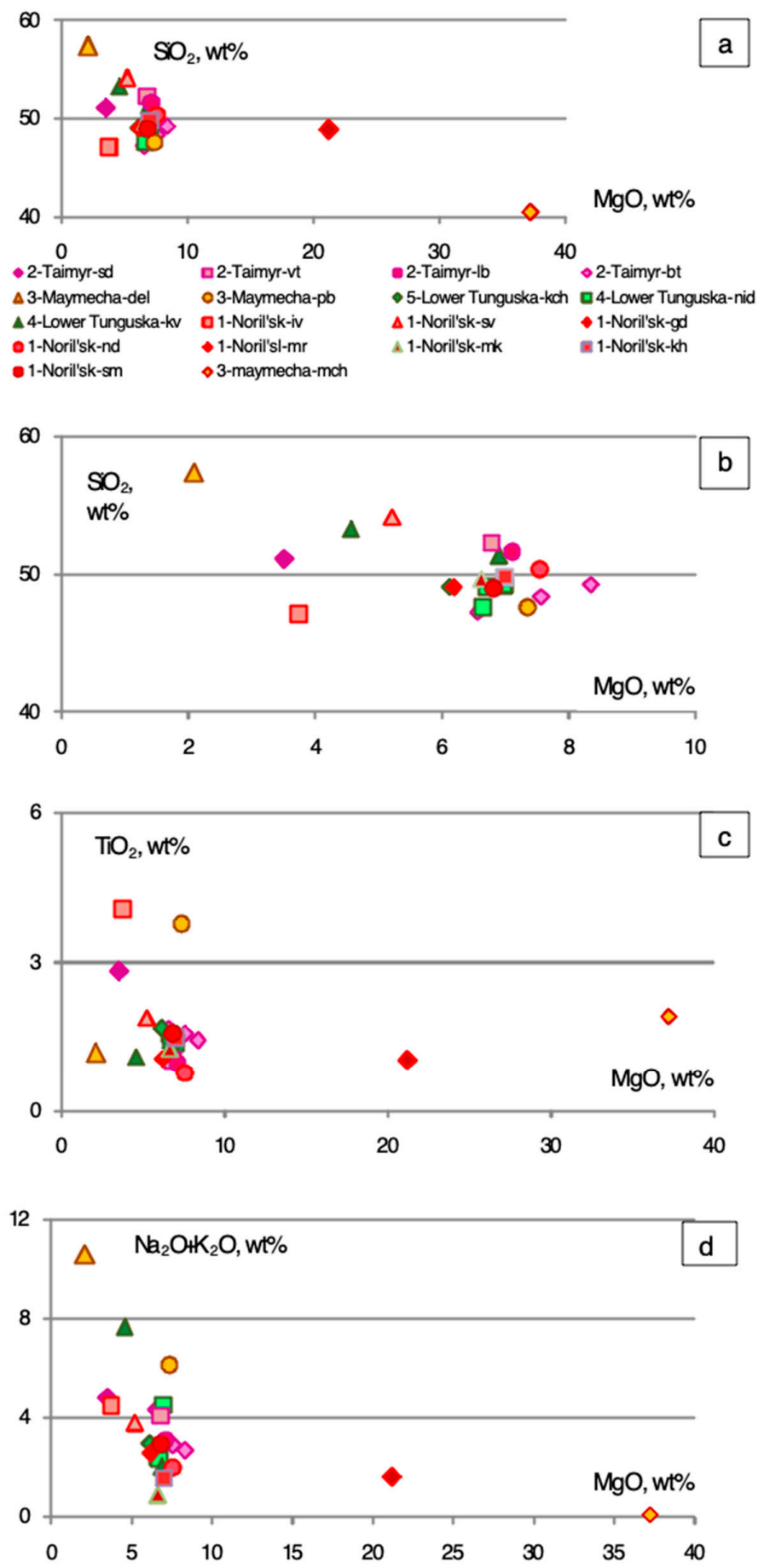

Figure 6. Diagrams of $\mathrm{MgO}-\mathrm{SiO}_{2}(\mathbf{a}, \mathbf{b}), \mathrm{TiO}_{2}(\mathbf{c}), \mathrm{Na}_{2} \mathrm{O}+\mathrm{K}_{2} \mathrm{O}$ (d) for volcanic rocks of the Siberian trap province. a, $\mathrm{SiO}_{2}-\mathrm{MgO}$ for all studied rocks, $\mathrm{b}$, part of diagram shown on Figure 4a. Formations: sd-Syradasaysky, vt-Verkhentamsky, lb-Labaksky, bt-Betlingsky, del—Delkansky, kch-Kochechumsky, nid-Nidymsky, kv-Korvunchansky, iv-Ivakinsky, sv—Syverminsky, gd-Gudchikhinsky, nd-Nadezhdinsky, mr-Morongovsky, mk-Mokulaevsky, khr-Kharaelakhsky, sm-Samoedsky. 


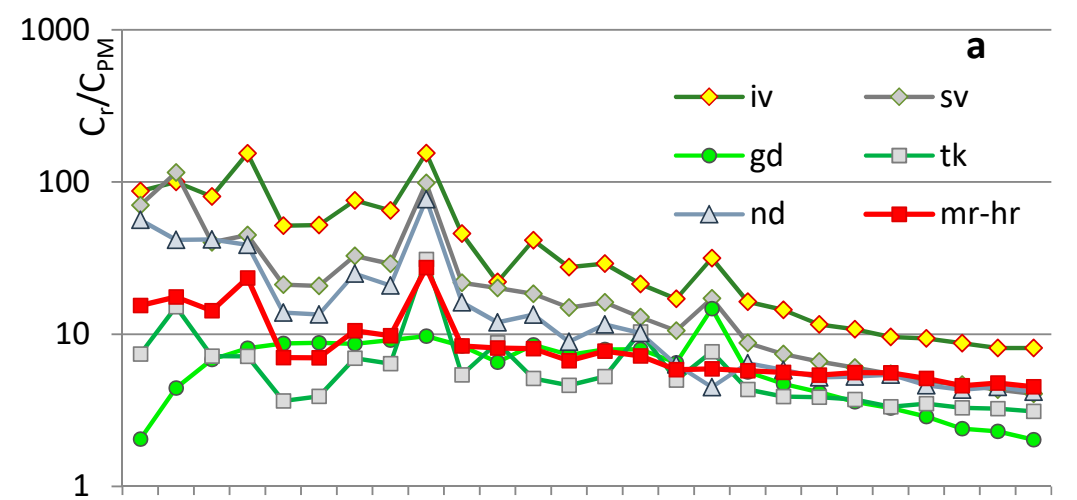

Rb Ba Th U Nb Ta La Ce Pb Pr Sr NdSm Zr Hf Eu Ti Gd Tb Dy Ho Y Er Yb Tm Lu

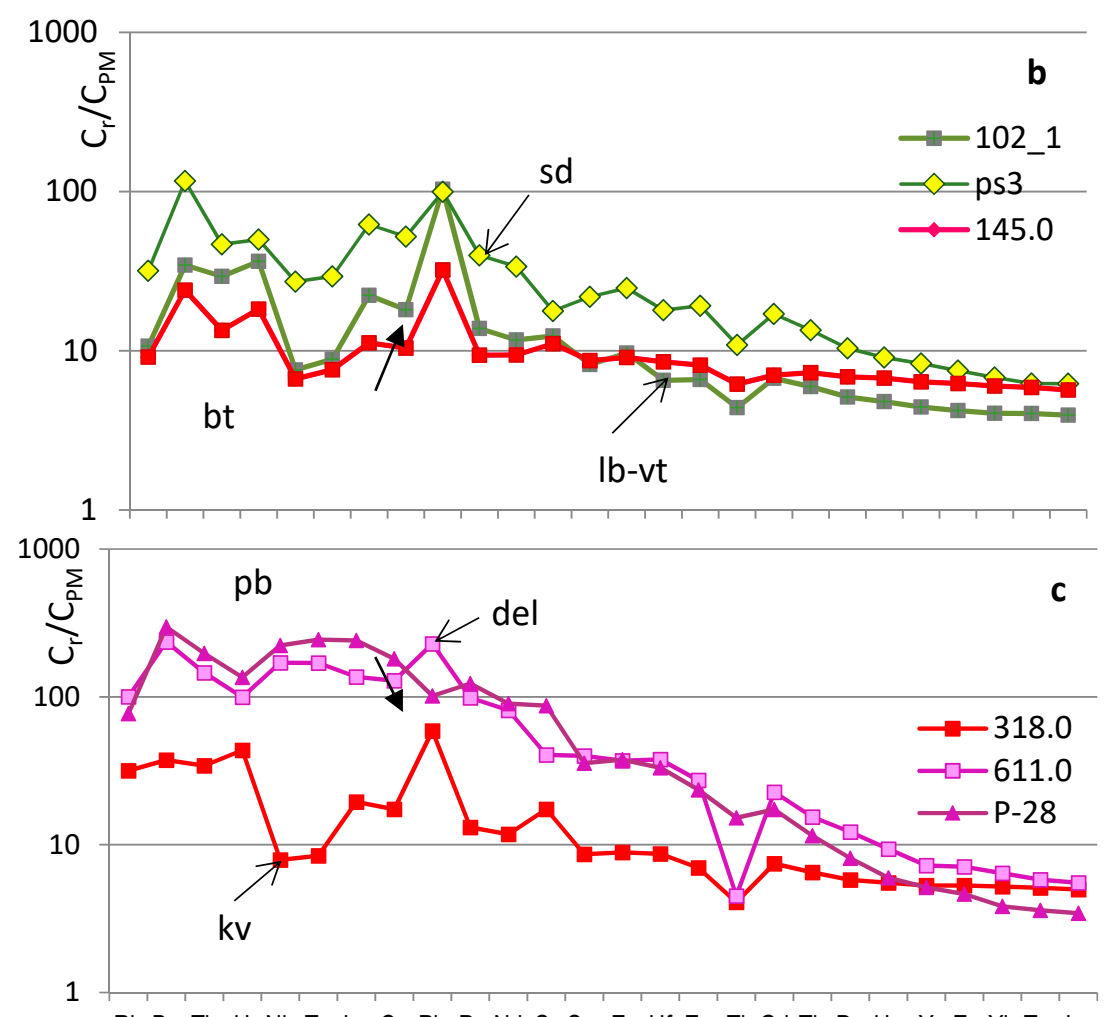

$\mathrm{Rb}$ Ba Th U Nb Ta La Ce Pb Pr Nd Sr Sm Zr Hf Eu Ti Gd Tb Dy Ho Y Er Yb Tm Lu

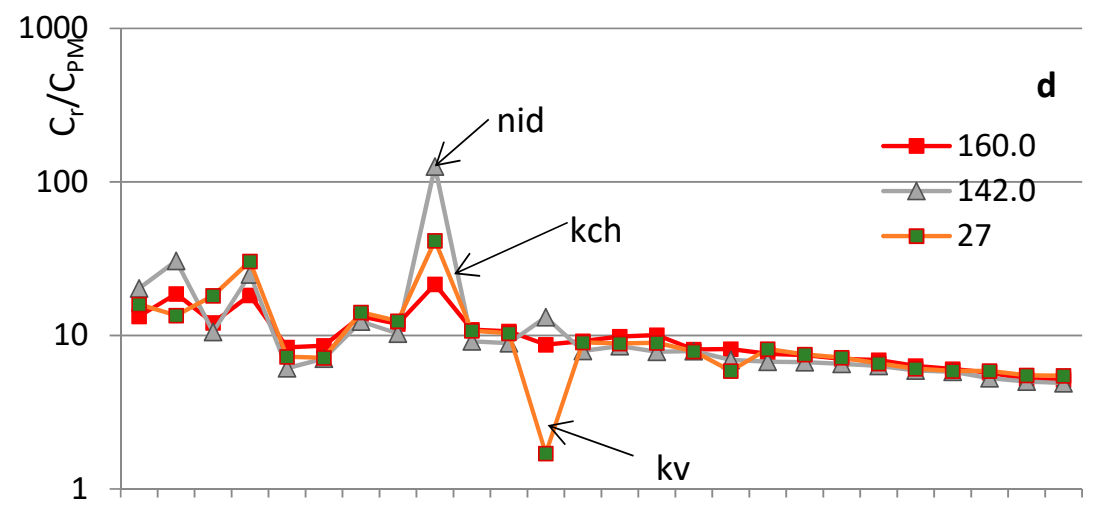

Rb Ba Th U Nb Ta La Ce Pb Pr Nd Sr Sm Zr Hf Eu Ti Gd Tb Dy Ho Y Er Yb Tm Lu

Figure 7. Representative spider diagrams for volcanic rocks of the Siberian trap province. Here and in Figure 6-normalized to Primitive Mantle, after [91]. Volcanic rocks, areas: (a) Noril'sk (1, number of area in Figure 1b), (b) Taimyr (2), (c) Maymecha-Kotuy (3), (d) r. Lower Tunguska valley (5). Data in Figure 7a are shown after [14]; data in Figure $7 \mathrm{~b}-\mathrm{d}$ are given in Table 3. Formations: iv-Ivakinsky, sv-Syverminsky, gd-Gudchikhinsky, tk-Tuklonsky, nd-Nadezhdinsky, mr-Morongovsky, khr-Kharaelakhsky, lb—Labaksky, vt-Verkhenetamsky, del-Delkansky, pb-Pravoboyarsky, bt-Betlinsky, nid-Nidymsky, kch-Kochechumsky, kv-Korvunchansky. 
Subalkaline and alkaline rock varieties are also common in the north of the province (1-3 areas). The maximum $\mathrm{Na}_{2} \mathrm{O}+\mathrm{K}_{2} \mathrm{O}$ content reaches $11 \mathrm{wt} \%$ in the Delkansky Formation of the Maymecha area (Figures 4 and 6d), as well as in the above-mentioned volcanic rocks with a high content of titanium: Syradasaysky (the Taimyr peninsula), Ivkansky, and Syverminsky formations (the Noril'sk region). Within the Tunguska syneclise elevated concentrations of these elements are established only in tuff of the Korvunchansky formation (Table 3).

Thus, the compositions of effusive rock are most diverse in the north areas of the Siberian trap province, the Noril'sk-Maymecha and Taimyr areas in comparison with the Tunguska and Angara-Taseeva synclines.

\subsection{Intrusive Rocks}

The distribution of intrusive rocks shows a dependence on position within the Siberian province and a correspondence to the composition of spatially associated lavas (see Figures 6-10).

For the first time we present geochemical data on intrusive bodies located in other parts of the Siberian trap province outside the Noril'sk area: the Taimyr Peninsula, the Kulyumber river valley, the central part of the Tunguska syneclise (Lower Tunguska river valley) and the south part of the Siberian platform (the Angara river valley) and compare them with the Noril'sk ore-bearing intrusions (Table 4, Figure $8 \mathrm{a}-\mathrm{d})$.

The intrusions of the Noril'sk and Taimyr areas are subdivided into four types that generally correlate with the lavas of this territory: (1) The subalkaline bodies of the Ergalakhsky intrusive complex (trachydolerite) which corresponds to the Ivakinsky Formation in composition and reversed polarity [92]. They form a chain of intrusions extending within the Noril'sk-Igarka paleorift zone for $500 \mathrm{~km}$. (2) The intrusions that are similar to the Gudchikhinsky formation are mostly located in the Taimyr peninsula (the Dyumtaleysky, Luktakhsky massifs, and several sill-like bodies) and have not been identified in the Noril'sk-Igarka area yet. These huge bodies (up to $60 \mathrm{~km}$ in length and $600 \mathrm{~m}$ thick) represent layered intrusions and consist of vehrlite, lherzolite, olivine, and olivine-free gabbro with elevated titanomagnetite content reaching up to $90 \mathrm{vol} \%$ and forming $\mathrm{Fe}-\mathrm{Ti}-\mathrm{V}$ ore. These rocks have the primitive composition of the parental magma and are enriched in Ti (Figures $8 b$ and 9a). Nevertheless, they contain economic PGE-Cu-Ni mineralization which is similar to the Noril'sk ores in mineralogy and isotopic composition $[16,67,69,70,93]$. (3) The Lower Talnakh complex, often regarded as an intrusive type within the Noril'sk complex (similar to the Nadezhdinsky Formation in geochemistry), combines many bodies of different shapes and sizes. The largest Binyudinsky massif was discovered in Taimyr and has been described in some publications [69,70,94]. In the Noril'sk area, this complex comprises the large Lower Talnakh, Zelenaya Griva (Table 4), and Klukvenny intrusions and some small intrusive bodies in the southern part of the territory. (4) The Noril'sk intrusive complex comprises many differentiated massifs (chonolites) of normal alkalinity and is close to the Morongovsky Formation according to its geochemistry and paleomagnetic data [85], although it is characterized by higher $\mathrm{MgO}$ content (10-12 wt \%) (Figure 6). Three intrusive bodies contain rich PGE-Cu-Ni massive and disseminated sulfide ores (i.e., Kharaelakh, Talnakh, and Noril'sk 1), whereas many others are barren or include only disseminated and small veins of massive ore (i.e., Vologochansky, Bolshaya Bariernaya, Maslovsky, Chernogorsky, and Noril'sk 2). The Lower Talnakh, Zelenaya Griva, and Chernogorsky massifs were studied in detail. The representative analyses of rocks from mentioned intrusive complexes, are given in Table 4.

The intrusions of the Kulyumber river valley are located $150 \mathrm{~km}$ to the south of Noril'sk and are related to the same intrusive complexes as the intrusions of the Noril'sk and Tunguska areas. They belong to the Ergalakhsky and Noril'sk complexes of the Noril'sk region as well as the Katangsky and Kureysky [36] which are widespread in the Tunguska syncline. The intrusions are localized near the zone of the large Lamsko-Letninsky fault (Figure 1b), mostly in Devonian-Permian rocks. Thus, their structural position is similar to the Noril'sk ore-bearing massifs. The only one mineralized intrusion was discovered within Silurian sedimentary deposits and was called "the Siluriyskaya" 
(unpublished data); it is differentiated from troctolite to leucogabbro. The major part of intrusive bodies $(>80 \%)$ vary in thickness from a few meters to $100 \mathrm{~m}$ and extend over a few kilometers. They consist of gabbro-dolerites without visual differentiation. Most of the intrusive bodies are identical in geochemistry. They correspond to gabbro (46.99-51.17 $\mathrm{wt} \% \mathrm{SiO}_{2}$ ) with elevated titanium contents (1.20-1.56 wt \% $\mathrm{TiO}_{2}$. Figure 8c) and record the predominance of sodium over potassium. According to their chemical composition they can be attributed to the Katangsky complex extending across the Siberian platform (Table 4).

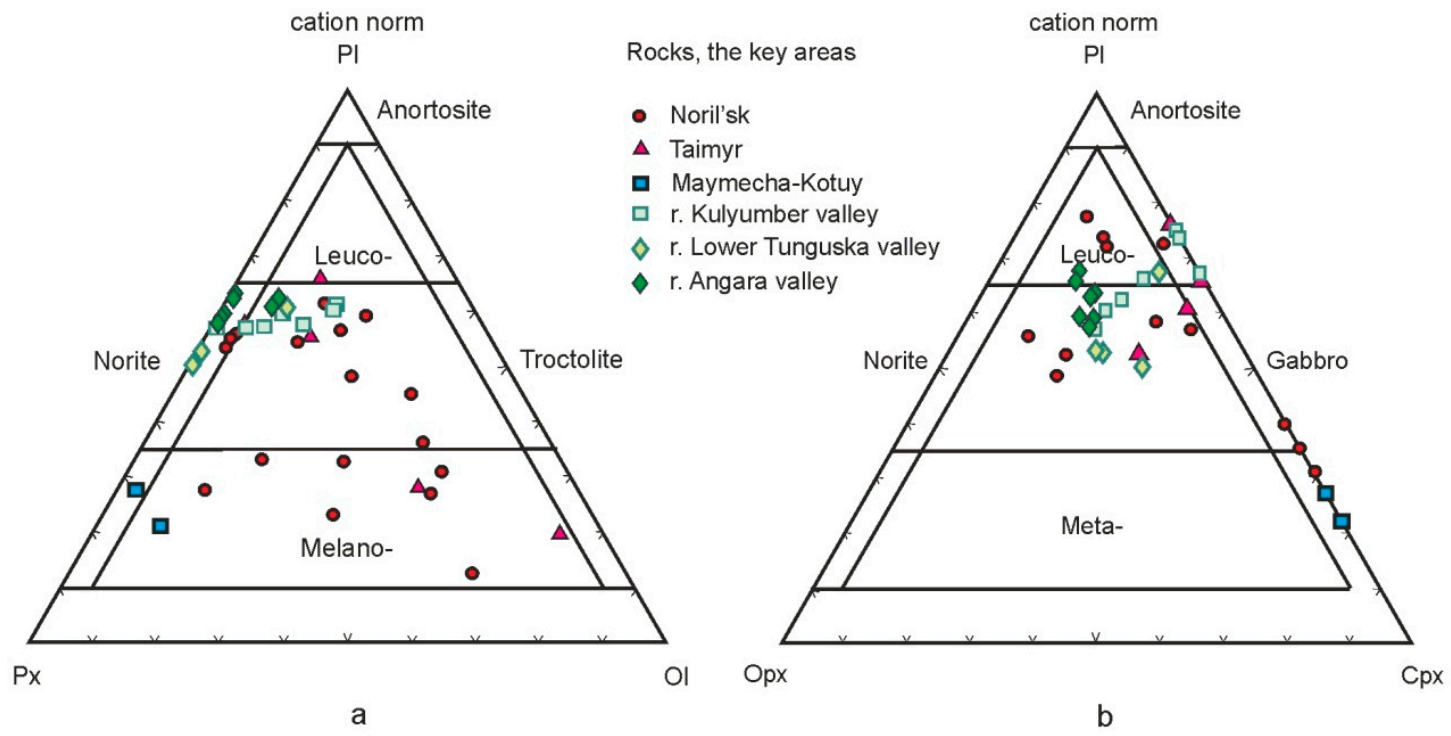

Figure 8. Diagrams of $\mathrm{Px}-\mathrm{Ol}-\mathrm{Pl}(\mathbf{a})$ and $\mathrm{Cpx}-\mathrm{Opx}-\mathrm{Pl}(\mathbf{b})$ for intrusive rocks of the Siberian trap province 1-6 analyses from the key areas: 1, Noril'sk; 2, Taimyr; 3, Maymecha-Kotuy; 4,r. Kulyumber;5, Lower Tunguska, 6, r. Angara (see Figure 2b). Data in Table 4. The calculation of CIPW and the construction of triangles was carried out with the IGPET v.200p Terra Softa Inc. package. The calculation was carried out with the separation of $\mathrm{Fe}_{2} \mathrm{O}_{3}$ and $\mathrm{FeO}$ after [95]. IUGS diagrams were used for basic and ultrabasic intrusive rocks.

Table 4. Chemical composition of the intrusive rocks from the Siberian trap province.

\begin{tabular}{|c|c|c|c|c|c|c|c|c|}
\hline No & 1 & 2 & 3 & 4 & 5 & 6 & 7 & 8 \\
\hline $\begin{array}{c}\mathrm{N} \\
\text { sample }\end{array}$ & LP-5/369 & LP-1/1542 & LP-1/1279 & LP-1-1510 & 3 LP-1-1413 & 3 OP4/1938.7 & 7 OP4/2017.4 & 4 OP4/1984 \\
\hline $\mathrm{SiO}_{2}$ & 45.17 & 47.3 & 36.81 & 35.53 & 43.61 & 44.66 & 41.91 & 45.15 \\
\hline $\mathrm{TiO}_{2}$ & 3.36 & 0.72 & 5.22 & 2.89 & 0.96 & 0.41 & 0.42 & 0.57 \\
\hline $\mathrm{Al}_{2} \mathrm{O}_{3}$ & 12.67 & 19.53 & 14.74 & 5.37 & 7.62 & 13.73 & 7.50 & 14.96 \\
\hline $\mathrm{Fe}_{2} \mathrm{O}_{3}$ & 15.09 & 7.43 & 21.78 & 27.75 & 17.20 & 11.72 & 14.16 & 11.89 \\
\hline $\mathrm{MnO}$ & 0.179 & 0.111 & 0.146 & 0.34 & 0.29 & 0.19 & 0.29 & 0.26 \\
\hline $\mathrm{MgO}$ & 5.46 & 7.44 & 6.01 & 22.87 & 21.74 & 15.93 & 23.18 & 13.91 \\
\hline $\mathrm{CaO}$ & 10.91 & 13.36 & 11.57 & 3.55 & 6.85 & 9.44 & 5.28 & 9.30 \\
\hline $\mathrm{Na}_{2} \mathrm{O}$ & 2.92 & 2.58 & 1.97 & 0.88 & 1.26 & 1.36 & 0.96 & 1.13 \\
\hline $\mathrm{K}_{2} \mathrm{O}$ & 0.63 & 0.28 & 0.19 & 0.21 & 0.11 & 0.76 & 0.36 & 0.81 \\
\hline $\mathrm{P}_{2} \mathrm{O}_{5}$ & 0.36 & 0.17 & 0.21 & 0.17 & 0.05 & 0.05 & 0.07 & 0.04 \\
\hline LOI & 2.87 & 0.1 & 0.51 & 0.34 & 0.88 & 2.67 & 6.03 & 2.70 \\
\hline Total & 99.62 & 99.02 & 99.16 & 99.90 & 100.57 & 100.91 & 100.16 & 100.73 \\
\hline $\mathrm{Rb}$ & 18 & 2.93 & 2.45 & 5.74 & 2.72 & 32.2 & 11.2 & 28.1 \\
\hline $\mathrm{Sr}$ & 438 & 624 & 738 & 174 & 259 & 222 & 116 & 270 \\
\hline Y & 31 & 9.87 & 12.64 & 10.90 & 10.22 & 12.20 & 9.70 & 9.65 \\
\hline $\mathrm{Ba}$ & 229 & 120 & 81 & 88 & 75 & 271 & 75 & 378 \\
\hline $\mathrm{La}$ & 20 & 5.13 & 6.38 & 8.25 & 4.37 & 6.94 & 4.20 & 6.57 \\
\hline
\end{tabular}


Table 4. Cont.

\begin{tabular}{|c|c|c|c|c|c|c|c|c|}
\hline $\mathrm{Ce}$ & 51 & 12.0 & 17 & 18.6 & 9.55 & 14.4 & 8.96 & 11.8 \\
\hline $\operatorname{Pr}$ & 6.88 & 1.74 & 2.69 & 2.45 & 1.35 & 1.73 & 1.18 & 1.36 \\
\hline $\mathrm{Nd}$ & 30.4 & 8.05 & 13.38 & 11.3 & 6.70 & 6.48 & 5.93 & 6.24 \\
\hline $\mathrm{Sm}$ & 7.82 & 2.20 & 3.72 & 2.64 & 2.02 & 1.65 & 1.45 & 1.41 \\
\hline $\mathrm{Eu}$ & 2.66 & 0.97 & 1.34 & 0.80 & 0.61 & 0.64 & 0.24 & 0.60 \\
\hline $\mathrm{Gd}$ & 8.27 & 2.30 & 3.77 & 2.64 & 2.21 & 1.87 & 1.46 & 1.39 \\
\hline $\mathrm{Tb}$ & 1.30 & 0.34 & 0.52 & 0.38 & 0.33 & 0.31 & 0.25 & 0.25 \\
\hline Dy & 6.77 & 1.99 & 2.87 & 2.21 & 2.20 & 1.77 & 1.71 & 1.57 \\
\hline Ho & 1.29 & 0.37 & 0.50 & 0.41 & 0.38 & 0.43 & 0.40 & 0.35 \\
\hline Er & 3.32 & 0.95 & 1.23 & 1.09 & 1.09 & 1.18 & 0.93 & 0.88 \\
\hline $\mathrm{Tm}$ & 0.45 & 0.13 & 0.15 & 0.15 & 0.13 & 0.16 & 0.14 & 0.12 \\
\hline $\mathrm{Yb}$ & 2.73 & 0.76 & 0.86 & 1.01 & 0.95 & 1.04 & 0.92 & 0.86 \\
\hline $\mathrm{Lu}$ & 0.37 & 0.11 & 0.12 & 0.15 & 0.14 & 0.16 & 0.12 & 0.12 \\
\hline $\mathrm{Pb}$ & 2.77 & 2.80 & 2.00 & 14.35 & 1.03 & 13.7 & 7.76 & 21.8 \\
\hline Th & 2.34 & 0.26 & 0.41 & 0.93 & 0.39 & 1.11 & 1.16 & 0.98 \\
\hline $\mathrm{U}$ & 0.66 & 0.10 & 0.14 & 0.30 & 0.11 & 0.44 & 0.41 & 0.29 \\
\hline Sc & 26 & 26.6 & 21.1 & 14.9 & 25.9 & & & \\
\hline $\mathrm{V}$ & 426 & 150 & 270 & & & 144 & 123 & 139 \\
\hline $\mathrm{Cr}$ & 73 & & & & & 345 & 187 & 288 \\
\hline $\mathrm{Co}$ & 47 & 51.1 & 93 & 74 & 33.7 & 105 & 93 & 68 \\
\hline $\mathrm{Ni}$ & 91 & 341 & 611 & 1322 & 1094 & 561 & 602 & 334 \\
\hline $\mathrm{Cu}$ & 204 & 314 & 841 & 223 & 198 & 323 & 119 & 116 \\
\hline $\mathrm{Zn}$ & 169 & 54 & 154 & 441 & 75.9 & 187 & 154 & 191 \\
\hline $\mathrm{Zr}$ & 165 & 34 & 64 & 74.6 & 33.7 & 39 & 42 & 40 \\
\hline $\mathrm{Nb}$ & 21 & 2.18 & 5.03 & 6.75 & 4.65 & 3.92 & 3.22 & 3.99 \\
\hline $\mathrm{Hf}$ & 4.85 & 1.06 & 2.06 & 1.91 & 1.12 & 1.09 & 1.06 & 0.96 \\
\hline $\mathrm{Ta}$ & 3.5 & 0.14 & 0.37 & 0.44 & 0.30 & 0.17 & 0.17 & 0.18 \\
\hline No & 9 & 10 & 11 & 12 & 13 & 14 & 15 & 16 \\
\hline $\begin{array}{c}\mathrm{N} \\
\text { sample }\end{array}$ & OP4/1990. & F233/374 & F233/336.1 & F233/359.4 & F233/379.2 & Ch55/73.2 & Ch-55/96.9 & Ch-55/129. \\
\hline $\mathrm{SiO}_{2}$ & 45.26 & 44.85 & 45.26 & 41.80 & 47.71 & 50.02 & 48.97 & 46.60 \\
\hline $\mathrm{TiO}_{2}$ & 0.44 & 0.60 & 0.55 & 0.41 & 1.01 & 1.00 & 0.67 & 0.48 \\
\hline $\mathrm{Al}_{2} \mathrm{O}_{3}$ & 9.86 & 10.63 & 11.15 & 6.69 & 15.33 & 15.49 & 16.60 & 16.93 \\
\hline $\mathrm{Fe}_{2} \mathrm{O}_{3}$ & 13.93 & 14.21 & 7.46 & 13.73 & 11.97 & 9.39 & 8.42 & 9.29 \\
\hline $\mathrm{MnO}$ & 0.29 & 0.33 & 0.16 & 0.22 & 0.24 & 0.13 & 0.10 & 0.16 \\
\hline $\mathrm{MgO}$ & 18.05 & 17.15 & 18.55 & 25.85 & 8.87 & 6.77 & 8.19 & 10.70 \\
\hline $\mathrm{CaO}$ & 7.00 & 8.18 & 11.74 & 7.24 & 8.65 & 14.11 & 14.35 & 12.65 \\
\hline $\mathrm{Na}_{2} \mathrm{O}$ & 1.02 & 1.87 & 2.03 & 1.31 & 1.52 & 2.16 & 1.78 & 1.32 \\
\hline $\mathrm{K}_{2} \mathrm{O}$ & 0.88 & 0.92 & 1.33 & 0.97 & 1.40 & 0.89 & 0.30 & 0.41 \\
\hline $\mathrm{P}_{2} \mathrm{O}_{5}$ & 0.05 & 0.08 & 0.04 & 0.06 & 0.11 & 0.10 & 0.09 & 0.06 \\
\hline LOI & 4.21 & 2.16 & 2.17 & 2.62 & 3.74 & 0.84 & 1.25 & 1.88 \\
\hline Total & 100.98 & 100.97 & 100.43 & 100.90 & 100.54 & 100.89 & 100.74 & 100.49 \\
\hline $\mathrm{Rb}$ & 28.7 & 30.4 & 46.9 & 28.2 & 35.3 & 24.9 & 6.4 & 9.6 \\
\hline $\mathrm{Sr}$ & 158 & 211 & 157 & 112 & 319 & 399 & 284 & 310 \\
\hline $\mathrm{Y}$ & 11.46 & 13.0 & 12.7 & 11.4 & 17.9 & 19.1 & 13.2 & 9.8 \\
\hline $\mathrm{Ba}$ & 156 & 135 & 65 & 94 & 314 & 146 & 85 & 113 \\
\hline $\mathrm{La}$ & 5.47 & 7.5 & 3.0 & 2.8 & 8.7 & 4.30 & 3.32 & 3.20 \\
\hline $\mathrm{Ce}$ & 12.3 & 14.9 & 9.1 & 9.8 & 22.2 & 11.0 & 8.04 & 6.95 \\
\hline $\mathrm{Pr}$ & 1.42 & 1.74 & 1.52 & 1.48 & 2.81 & 1.67 & 1.15 & 0.97 \\
\hline $\mathrm{Nd}$ & 5.51 & 8.03 & 7.43 & 6.24 & 13.7 & 8.57 & 5.61 & 4.59 \\
\hline $\mathrm{Sm}$ & 1.46 & 2.00 & 1.93 & 1.70 & 3.32 & 2.54 & 1.68 & 1.28 \\
\hline $\mathrm{Eu}$ & 0.40 & 0.54 & 0.29 & 0.26 & 0.87 & 0.88 & 0.65 & 0.52 \\
\hline Gd & 1.72 & 2.02 & 2.01 & 2.05 & 3.03 & 3.09 & 2.05 & 1.56 \\
\hline $\mathrm{Tb}$ & 0.28 & 0.35 & 0.33 & 0.32 & 0.56 & 0.53 & 0.35 & 0.26 \\
\hline Dy & 1.53 & 2.21 & 2.26 & 1.81 & 3.20 & 3.50 & 2.39 & 1.78 \\
\hline Ho & 0.39 & 0.51 & 0.49 & 0.41 & 0.74 & 0.71 & 0.48 & 0.36 \\
\hline Er & 1.10 & 1.30 & 1.20 & 1.19 & 1.93 & 2.09 & 1.37 & 1.10 \\
\hline $\mathrm{Tm}$ & 0.14 & 0.18 & 0.18 & 0.17 & 0.25 & 0.29 & 0.19 & 0.14 \\
\hline $\mathrm{Yb}$ & 1.03 & 1.27 & 1.14 & 1.03 & 1.76 & 1.93 & 1.38 & 1.05 \\
\hline $\mathrm{Lu}$ & 0.16 & 0.18 & 0.17 & 0.17 & 0.23 & 0.29 & 0.21 & 0.16 \\
\hline $\mathrm{Pb}$ & 3.93 & 12.3 & 23.5 & 6.57 & 18.8 & 1.43 & 6.61 & 5.88 \\
\hline Th & 1.14 & 1.58 & 0.80 & 0.93 & 2.44 & 0.72 & 0.56 & 0.40 \\
\hline
\end{tabular}


Table 4. Cont.

\begin{tabular}{|c|c|c|c|c|c|c|c|c|}
\hline $\mathrm{U}$ & 0.36 & 0.57 & 0.19 & 0.18 & 0.74 & 0.23 & 0.22 & 0.14 \\
\hline $\mathrm{Sc}$ & & & & & & 51.0 & 43.5 & 32.7 \\
\hline $\mathrm{V}$ & 116 & 155 & 138 & 109 & 1077 & & & \\
\hline $\mathrm{Cr}$ & 212 & 174 & 134 & 120 & 209 & & & \\
\hline $\mathrm{Co}$ & 78 & 82 & 51 & 104 & 47 & 43 & 48 & 31 \\
\hline $\mathrm{Ni}$ & 460 & 457 & 685 & 857 & 253 & 84 & 146 & 262 \\
\hline $\mathrm{Cu}$ & 25 & 200 & 618 & 185 & 488 & 93 & 168 & 139 \\
\hline $\mathrm{Zn}$ & 95 & 200 & 221 & 167 & 732 & 45 & 75 & 304 \\
\hline $\mathrm{Zr}$ & 34 & 54 & 43 & 35 & 77 & 43 & 48 & 31 \\
\hline $\mathrm{Nb}$ & 3.53 & 4.9 & 7.3 & 8.8 & 7.8 & 2.95 & 1.98 & 1.56 \\
\hline Hf & 0.90 & 1.4 & 1.2 & 1.0 & 2.0 & 1.24 & 1.17 & 0.82 \\
\hline Ta & 0.14 & 0.2 & 5.5 & 5.8 & 5.5 & 0.18 & 0.11 & 0.10 \\
\hline No & 17 & 18 & 19 & 20 & 21 & 22 & 23 & 24 \\
\hline $\begin{array}{c}\mathrm{N} \\
\text { sample }\end{array}$ & Ch-55/16 & Ch-55/19 & Ch-55/21 & Ch-55/25 & $.5 \mathrm{Ch}-55 / 269$ & 5 Ch-55/275.1 & Kul-6 & Kul-21 \\
\hline $\mathrm{SiO}_{2}$ & 45.61 & 46.48 & 45.67 & 41.42 & 45.42 & 46.06 & 47.56 & 47.48 \\
\hline $\mathrm{TiO}_{2}$ & 0.51 & 0.60 & 0.60 & 0.69 & 0.82 & 0.77 & 1.31 & 1.52 \\
\hline $\mathrm{Al}_{2} \mathrm{O}_{3}$ & 18.39 & 19.06 & 17.21 & 9.14 & 11.80 & 10.70 & 14.64 & 14.98 \\
\hline $\mathrm{Fe}_{2} \mathrm{O}_{3}$ & 9.88 & 10.02 & 11.13 & 16.68 & 10.09 & 7.03 & 12.87 & 12.62 \\
\hline $\mathrm{MnO}$ & 0.15 & 0.12 & 0.09 & 0.25 & 0.33 & 0.07 & 0.18 & 0.17 \\
\hline $\mathrm{MgO}$ & 11.97 & 9.48 & 10.94 & 21.08 & 12.71 & 13.65 & 7.25 & 6.87 \\
\hline $\mathrm{CaO}$ & 10.31 & 11.63 & 10.78 & 6.13 & 15.47 & 19.67 & 9.78 & 8.91 \\
\hline $\mathrm{Na}_{2} \mathrm{O}$ & 1.56 & 1.59 & 1.54 & 0.86 & 1.38 & 1.25 & 3.41 & 3.97 \\
\hline $\mathrm{K}_{2} \mathrm{O}$ & 0.46 & 0.48 & 0.50 & 0.34 & 0.59 & 0.20 & 0.39 & 0.67 \\
\hline $\mathrm{P}_{2} \mathrm{O}_{5}$ & 0.08 & 0.06 & 0.08 & 0.09 & 0.12 & 0.12 & 0.14 & 0.19 \\
\hline LOI & 2.04 & 1.41 & 1.94 & 2.92 & 2.90 & 1.01 & 2.40 & 2.53 \\
\hline Total & 100.96 & 100.92 & 100.50 & 99.60 & 101.63 & 100.52 & 99.93 & 99.91 \\
\hline $\mathrm{Rb}$ & 9.8 & 12.3 & 13.9 & 7.9 & 13.8 & 4.8 & 8.6 & 15.1 \\
\hline $\mathrm{Sr}$ & 344 & 335 & 332 & 138 & 267 & 302 & 218 & 228 \\
\hline $\mathrm{Y}$ & 10.1 & 11.7 & 10.8 & 12.1 & 24.2 & 22.3 & 23.2 & 28.3 \\
\hline $\mathrm{Ba}$ & 141 & 99 & 93 & 115 & 162 & 37 & 117 & 156 \\
\hline $\mathrm{La}$ & 3.82 & 3.60 & 3.35 & 3.95 & 7.36 & 11.3 & 6.83 & 7.75 \\
\hline $\mathrm{Ce}$ & 8.63 & 8.30 & 7.93 & 8.70 & 19.3 & 25.6 & 16.2 & 19.3 \\
\hline Pr & 1.17 & 1.15 & 1.11 & 1.18 & 2.77 & 3.36 & 2.22 & 2.76 \\
\hline $\mathrm{Nd}$ & 5.47 & 5.53 & 5.30 & 5.58 & 13.60 & 15.66 & 10.6 & 12.8 \\
\hline $\mathrm{Sm}$ & 1.47 & 1.52 & 1.41 & 1.51 & 3.67 & 4.08 & 3.06 & 3.79 \\
\hline $\mathrm{Eu}$ & 0.57 & 0.59 & 0.57 & 0.56 & 1.18 & 0.90 & 1.07 & 1.24 \\
\hline $\mathrm{Gd}$ & 1.65 & 1.87 & 1.69 & 1.84 & 4.16 & 4.13 & 3.34 & 4.12 \\
\hline $\mathrm{Tb}$ & 0.28 & 0.30 & 0.29 & 0.32 & 0.67 & 0.64 & 0.60 & 0.77 \\
\hline Dy & 1.84 & 2.06 & 1.91 & 2.15 & 4.35 & 4.12 & 3.98 & 5.01 \\
\hline Ho & 0.37 & 0.44 & 0.40 & 0.45 & 0.87 & 0.85 & 0.88 & 1.12 \\
\hline Er & 1.11 & 1.24 & 1.18 & 1.29 & 2.52 & 2.49 & 2.49 & 3.06 \\
\hline $\mathrm{Tm}$ & 0.14 & 0.18 & 0.17 & 0.19 & 0.34 & 0.35 & 0.35 & 0.46 \\
\hline $\mathrm{Yb}$ & 1.09 & 1.24 & 1.14 & 1.30 & 2.33 & 2.44 & 2.22 & 2.80 \\
\hline $\mathrm{Lu}$ & 0.16 & 0.19 & 0.18 & 0.20 & 0.35 & 0.38 & 0.34 & 0.41 \\
\hline $\mathrm{Pb}$ & 13.01 & 2.05 & 3.72 & 10.7 & 62.7 & 14.7 & 1.27 & 0.93 \\
\hline Th & 0.66 & 0.61 & 0.39 & 0.61 & 0.74 & 2.01 & 0.90 & 1.10 \\
\hline $\mathrm{U}$ & 0.24 & 0.25 & 0.15 & 0.24 & 0.32 & 1.62 & 0.38 & 0.75 \\
\hline Sc & 16.2 & 22.6 & 24.6 & 25.0 & 30.3 & 13.6 & 36.4 & 36.3 \\
\hline $\mathrm{V}$ & & & & & & & 286 & 294 \\
\hline $\mathrm{Cr}$ & & & & & & & 158 & 162 \\
\hline $\mathrm{Co}$ & 46 & 44 & 34 & 45 & 87 & 152 & 49.0 & 41.3 \\
\hline $\mathrm{Ni}$ & 332 & 262 & 309 & 2956 & 227 & 890 & 118 & 128 \\
\hline $\mathrm{Cu}$ & 91 & 112 & 182 & 4112 & 728 & 1941 & 147 & 135 \\
\hline $\mathrm{Zn}$ & 106 & 79 & 84 & 1179 & 853 & 553 & 88 & 53 \\
\hline $\mathrm{Zr}$ & 46 & 44 & 34 & 45 & 87 & 152 & 88 & 100 \\
\hline $\mathrm{Nb}$ & 1.94 & 1.97 & 1.94 & 2.04 & 2.30 & 5.65 & 3.61 & 4.52 \\
\hline $\mathrm{Hf}$ & 1.21 & 1.14 & 0.87 & 1.26 & 2.91 & 3.71 & 2.08 & 2.17 \\
\hline $\mathrm{Ta}$ & 0.12 & 0.13 & 0.12 & 0.13 & 0.17 & 0.44 & 0.22 & 0.30 \\
\hline
\end{tabular}


Table 4. Cont

\begin{tabular}{|c|c|c|c|c|c|c|c|c|}
\hline No & 25 & 26 & 27 & 28 & 29 & 30 & 31 & 32 \\
\hline $\begin{array}{c}\mathrm{N} \\
\text { sample }\end{array}$ & Kul-25 & Kul-28 & Kul-32 & Kul-40 & LT-158 & LT-151 & LT-189 & LT-215 \\
\hline $\mathrm{SiO}_{2}$ & 47.24 & 47.59 & 47.60 & 48.46 & 49.85 & 48.18 & 48.71 & 48.84 \\
\hline $\mathrm{TiO}_{2}$ & 1.91 & 1.31 & 1.56 & 1.42 & 0.94 & 1.74 & 1.17 & 1.40 \\
\hline $\mathrm{Al}_{2} \mathrm{O}_{3}$ & 14.51 & 14.75 & 14.59 & 14.98 & 13.02 & 13.26 & 15.47 & 13.71 \\
\hline $\mathrm{Fe}_{2} \mathrm{O}_{3}$ & 13.69 & 11.56 & 13.86 & 13.45 & 11.49 & 15.19 & 12.39 & 14.13 \\
\hline $\mathrm{MnO}$ & 0.18 & 0.16 & 0.19 & 0.20 & 0.18 & 0.23 & 0.15 & 0.20 \\
\hline $\mathrm{MgO}$ & 6.79 & 7.23 & 7.01 & 6.97 & 7.15 & 6.01 & 6.01 & 6.80 \\
\hline $\mathrm{CaO}$ & 10.07 & 10.99 & 10.16 & 10.34 & 12.22 & 9.82 & 10.64 & 11.10 \\
\hline $\mathrm{Na}_{2} \mathrm{O}$ & 3.03 & 3.45 & 2.68 & 2.56 & 2.14 & 2.26 & 2.91 & 2.17 \\
\hline $\mathrm{K}_{2} \mathrm{O}$ & 0.38 & 0.52 & 0.47 & 0.50 & 0.41 & 0.89 & 0.91 & 0.19 \\
\hline $\mathrm{P}_{2} \mathrm{O}_{5}$ & 0.17 & 0.18 & 0.19 & 0.15 & 0.09 & 0.20 & 0.11 & 0.13 \\
\hline LOI & 1.94 & 2.18 & 1.61 & 0.90 & 1.50 & 1.50 & 1.50 & 1.50 \\
\hline Total & 99.91 & 99.92 & 99.92 & 99.93 & 99.00 & 99.28 & 99.98 & 100.18 \\
\hline $\mathrm{Rb}$ & 14.7 & 10.8 & 12.7 & 13.3 & 10.2 & 22.0 & 29.9 & 3.2 \\
\hline $\mathrm{Sr}$ & 333 & 243 & 231 & 199 & 214 & 276 & 356 & 186 \\
\hline $\mathrm{Y}$ & 35.1 & 25.7 & 27.6 & 25.5 & 22.5 & 36.4 & 25.7 & 29.2 \\
\hline $\mathrm{Ba}$ & 148 & 110 & 140 & 112 & 205 & 659 & 158 & 127 \\
\hline $\mathrm{La}$ & 10.3 & 7.54 & 8.93 & 6.99 & 8.90 & 16.3 & 8.38 & 8.55 \\
\hline $\mathrm{Ce}$ & 25.2 & 18.4 & 20.9 & 16.8 & 19.4 & 35.6 & 19.2 & 20.7 \\
\hline $\operatorname{Pr}$ & 3.64 & 2.58 & 2.93 & 2.41 & 2.42 & 4.36 & 2.49 & 2.79 \\
\hline $\mathrm{Nd}$ & 17.0 & 12.3 & 13.5 & 11.3 & 11.3 & 19.8 & 12.0 & 13.6 \\
\hline $\mathrm{Sm}$ & 4.93 & 3.57 & 3.82 & 3.48 & 3.08 & 5.13 & 3.33 & 3.87 \\
\hline $\mathrm{Eu}$ & 1.62 & 1.16 & 1.27 & 1.19 & 1.09 & 1.68 & 1.20 & 1.35 \\
\hline $\mathrm{Gd}$ & 5.37 & 3.86 & 4.20 & 3.83 & 3.99 & 6.47 & 4.39 & 5.08 \\
\hline $\mathrm{Tb}$ & 0.99 & 0.71 & 0.77 & 0.69 & 0.65 & 1.03 & 0.71 & 0.82 \\
\hline Dy & 6.41 & 4.71 & 5.07 & 4.69 & 4.28 & 6.58 & 4.68 & 5.31 \\
\hline Ho & 1.35 & 1.05 & 1.08 & 1.00 & 0.88 & 1.35 & 0.98 & 1.11 \\
\hline Er & 3.70 & 2.78 & 2.98 & 2.80 & 2.60 & 4.17 & 3.04 & 3.31 \\
\hline $\mathrm{Tm}$ & 0.54 & 0.40 & 0.44 & 0.41 & 0.37 & 0.59 & 0.43 & 0.47 \\
\hline $\mathrm{Yb}$ & 3.53 & 2.49 & 2.85 & 2.65 & 2.54 & 3.95 & 2.81 & 3.12 \\
\hline $\mathrm{Lu}$ & 0.51 & 0.37 & 0.42 & 0.40 & 0.36 & 0.59 & 0.42 & 0.47 \\
\hline $\mathrm{Pb}$ & 0.88 & 0.93 & 1.73 & 2.11 & 1.61 & 2.72 & 1.37 & 1.73 \\
\hline Th & 1.41 & 0.98 & 1.08 & 0.79 & 1.28 & 2.35 & 1.50 & 1.30 \\
\hline $\mathrm{U}$ & 0.56 & 0.39 & 0.57 & 0.33 & 0.45 & 0.95 & 0.58 & 0.54 \\
\hline Sc & 50.0 & 36.6 & 37.0 & 36.8 & 42.7 & 32.5 & 35.6 & 36.4 \\
\hline $\mathrm{V}$ & 329 & 244 & 295 & 279 & 267 & 314 & 278 & 299 \\
\hline $\mathrm{Cr}$ & 248 & 181 & 157 & 168 & 83 & 106 & 76 & 123 \\
\hline Co & 54.4 & 40.8 & 48.7 & 47.1 & 42 & 44 & 39 & 47 \\
\hline $\mathrm{Ni}$ & 148 & 111 & 123 & 136 & 71 & 82 & 73 & 111 \\
\hline $\mathrm{Cu}$ & 104 & 76 & 179 & 225 & 91 & 153 & 100 & 155 \\
\hline $\mathrm{Zn}$ & 20 & 93 & 134 & 118 & 85 & 132 & 40 & 111 \\
\hline $\mathrm{Zr}$ & 118 & 82 & 109 & 88 & 79 & 142 & 87 & 104 \\
\hline $\mathrm{Nb}$ & 5.92 & 4.15 & 4.63 & 3.44 & 3.57 & 7.21 & 4.75 & 4.77 \\
\hline $\mathrm{Hf}$ & 2.77 & 1.90 & 2.26 & 1.72 & 2.09 & 3.77 & 2.47 & 2.80 \\
\hline Ta & 0.38 & 0.24 & 0.29 & 0.21 & 0.27 & 0.47 & 0.32 & 0.30 \\
\hline No & 33 & 34 & 35 & 36 & 37 & 38 & 39 & 40 \\
\hline $\begin{array}{c}\mathrm{N} \\
\text { sample }\end{array}$ & $1.1 \mathrm{~T}$ & $1.2 \mathrm{~T}$ & $1.3 \mathrm{~T}$ & $1.4 \mathrm{~T}$ & 1.1БП & $1.2 Б П$ & 1.ЗБП & 28 \\
\hline $\mathrm{SiO}_{2}$ & 48.55 & 49.34 & 49.06 & 49.33 & 47.34 & 47.54 & 47.47 & 47.23 \\
\hline $\mathrm{TiO}_{2}$ & 1.62 & 1.74 & 1.49 & 2.12 & 1.60 & 1.50 & 1.58 & 2.74 \\
\hline $\mathrm{Al}_{2} \mathrm{O}_{3}$ & 15.81 & 15.31 & 16.75 & 15.48 & 16.20 & 16.49 & 16.59 & 13.53 \\
\hline $\mathrm{Fe}_{2} \mathrm{O}_{3}$ & 13.76 & 13.28 & 13.00 & 14.51 & 14.82 & 14.56 & 14.69 & 15.41 \\
\hline $\mathrm{MnO}$ & 0.19 & 0.19 & 0.19 & 0.21 & 0.20 & 0.19 & 0.19 & 0.21 \\
\hline $\mathrm{MgO}$ & 6.12 & 6.11 & 5.37 & 4.45 & 6.86 & 6.66 & 6.33 & 6.23 \\
\hline $\mathrm{CaO}$ & 10.32 & 10.52 & 10.81 & 9.76 & 9.58 & 9.56 & 9.57 & 9.07 \\
\hline $\mathrm{Na}_{2} \mathrm{O}$ & 2.40 & 2.35 & 2.46 & 2.64 & 2.41 & 2.44 & 2.49 & 2.63 \\
\hline $\mathrm{K}_{2} \mathrm{O}$ & 0.60 & 0.62 & 0.52 & 0.78 & 0.58 & 0.57 & 0.67 & 0.60 \\
\hline $\mathrm{P}_{2} \mathrm{O}_{5}$ & 0.24 & 0.22 & 0.20 & 0.31 & 0.22 & 0.22 & 0.25 & 0.18 \\
\hline LOI & 0.24 & 0.14 & 0.32 & 0.27 & 0.12 & 0.10 & 0.06 & 0.31 \\
\hline Total & 99.84 & 99.83 & 100.16 & 99.87 & 99.94 & 99.83 & 99.89 & 98.14 \\
\hline
\end{tabular}


Table 4. Cont.

\begin{tabular}{|c|c|c|c|c|c|c|c|c|}
\hline $\mathrm{Rb}$ & 12.8 & 13.1 & 10.1 & 16.1 & 11.8 & 12.0 & 13.5 & 15.3 \\
\hline $\mathrm{Sr}$ & 204 & 200 & 221 & 210 & 204 & 209 & 215 & 233 \\
\hline $\mathrm{Y}$ & 32.8 & 34.5 & 29.5 & 43.9 & 26.5 & 28.2 & 29.7 & 27.3 \\
\hline $\mathrm{Ba}$ & 139 & 145 & 131 & 183 & 146 & 144 & 155 & 169 \\
\hline $\mathrm{La}$ & 8.26 & 8.44 & 7.21 & 10.9 & 7.96 & 8.31 & 8.83 & 11.0 \\
\hline $\mathrm{Ce}$ & 20.6 & 21.1 & 17.9 & 27.1 & 19.7 & 20.7 & 21.5 & 25.2 \\
\hline $\operatorname{Pr}$ & 2.86 & 2.94 & 2.47 & 3.78 & 2.67 & 2.76 & 2.94 & 3.45 \\
\hline $\mathrm{Nd}$ & 14.6 & 14.9 & 12.8 & 19.0 & 13.4 & 14.1 & 14.8 & 15.8 \\
\hline $\mathrm{Sm}$ & 4.43 & 4.58 & 3.91 & 5.78 & 3.86 & 4.01 & 4.19 & 4.09 \\
\hline $\mathrm{Eu}$ & 1.49 & 1.47 & 1.40 & 1.76 & 1.31 & 1.35 & 1.40 & 1.42 \\
\hline $\mathrm{Gd}$ & 5.52 & 5.63 & 4.92 & 7.11 & 4.60 & 5.02 & 5.10 & 4.70 \\
\hline $\mathrm{Tb}$ & 0.92 & 0.93 & 0.82 & 1.16 & 0.74 & 0.77 & 0.83 & 0.82 \\
\hline Dy & 5.90 & 6.15 & 5.29 & 7.57 & 4.88 & 5.12 & 5.36 & 5.14 \\
\hline Ho & 1.21 & 1.29 & 1.12 & 1.58 & 0.99 & 1.01 & 1.09 & 1.10 \\
\hline $\mathrm{Er}$ & 3.67 & 3.80 & 3.29 & 4.79 & 2.99 & 3.10 & 3.24 & 3.09 \\
\hline $\mathrm{Tm}$ & 0.50 & 0.53 & 0.45 & 0.67 & 0.42 & 0.43 & 0.45 & 0.45 \\
\hline $\mathrm{Yb}$ & 3.41 & 3.68 & 3.11 & 4.41 & 2.77 & 2.89 & 3.06 & 2.87 \\
\hline $\mathrm{Lu}$ & 0.49 & 0.52 & 0.45 & 0.65 & 0.41 & 0.42 & 0.44 & 0.43 \\
\hline $\mathrm{Pb}$ & 2.44 & 2.39 & 2.27 & 3.27 & 2.51 & 2.95 & 3.15 & 2.31 \\
\hline Th & 1.21 & 1.24 & 1.01 & 1.61 & 1.19 & 1.27 & 1.30 & 1.28 \\
\hline $\mathrm{U}$ & 0.50 & 0.56 & 0.43 & 0.71 & 0.44 & 0.45 & 0.48 & 0.38 \\
\hline Sc & 37.1 & 41.7 & 37.7 & 38.5 & 28.4 & 29.7 & 30.2 & 20.9 \\
\hline $\mathrm{V}$ & 306 & 342 & 304 & 372 & 259 & 253 & 261 & 183 \\
\hline $\mathrm{Cr}$ & 225 & 296 & 148 & 78 & 140 & 142 & 140 & 27.5 \\
\hline $\mathrm{Co}$ & 47 & 45 & 42 & 41 & 60 & 60 & 55 & 57.72 \\
\hline $\mathrm{Ni}$ & 89 & 69 & 62 & 37 & 170 & 167 & 146 & 123 \\
\hline $\mathrm{Cu}$ & 175 & 189 & 181 & 244 & 150 & 165 & 156 & 174 \\
\hline $\mathrm{Zn}$ & 98 & 100 & 99 & 125 & 106 & 112 & 105 & 212 \\
\hline $\mathrm{Zr}$ & 97 & 114 & 96 & 146 & 100 & 106 & 113 & 73.6 \\
\hline $\mathrm{Nb}$ & 5.43 & 5.45 & 4.80 & 7.24 & 4.96 & 5.04 & 5.51 & 7.22 \\
\hline Hf & 2.81 & 3.37 & 2.82 & 4.21 & 2.85 & 2.99 & 3.24 & 1.92 \\
\hline $\mathrm{Ta}$ & 0.31 & 0.37 & 0.31 & 0.45 & 0.35 & 0.30 & 0.31 & 0.40 \\
\hline No & 41 & 42 & 43 & 44 & 45 & & & \\
\hline $\begin{array}{c}\mathrm{N} \\
\text { sample }\end{array}$ & IFG-10c * & IFG-189 * & IFG-19 * & BHVO-2 & LOD & & & \\
\hline $\mathrm{SiO}_{2}$ & 42.88 & 42.93 & 50.09 & 49.3 & 0.04 & & & \\
\hline $\mathrm{TiO}_{2}$ & 4.55 & 4.79 & 1.25 & 7.82 & 0.005 & & & \\
\hline $\mathrm{Al}_{2} \mathrm{O}_{3}$ & 10.01 & 5.97 & 15.58 & 13.14 & 0.02 & & & \\
\hline $\mathrm{Fe}_{2} \mathrm{O}_{3}$ & 14.12 & 15.13 & 11.64 & 12.18 & 0.005 & & & \\
\hline $\mathrm{MnO}$ & 0.22 & 0.25 & 0.2 & 0.153 & 0.002 & & & \\
\hline $\mathrm{MgO}$ & 8.57 & 12.42 & 7.18 & 6.88 & 0.007 & & & \\
\hline $\mathrm{CaO}$ & 14.85 & 15.85 & 11.2 & 11.14 & 0.003 & & & \\
\hline $\mathrm{Na}_{2} \mathrm{O}$ & 2.54 & 1.09 & 2.21 & 2.18 & 0.01 & & & \\
\hline $\mathrm{K}_{2} \mathrm{O}$ & 1.45 & 0.9 & 0.49 & 0.48 & 0.004 & & & \\
\hline $\mathrm{P}_{2} \mathrm{O}_{5}$ & 0.81 & 0.67 & 0.16 & 0.26 & 0.008 & & & \\
\hline LOI & 2.18 & 3.41 & 0.93 & & & & & \\
\hline Total & 102.18 & 103.41 & 100.93 & & & & & \\
\hline $\mathrm{Rb}$ & 40 & 48 & 18 & 9.14 & 0.18 & & & \\
\hline $\mathrm{Sr}$ & 1532 & 800 & 220 & 380 & 4.15 & & & \\
\hline $\mathrm{Y}$ & 28 & 13 & 31 & 25.6 & 0.05 & & & \\
\hline $\mathrm{Ba}$ & 1190 & 3600 & 192 & 131 & 1.7 & & & \\
\hline $\mathrm{La}$ & 124 & 105 & 8.1 & 15.1 & 0.1 & & & \\
\hline $\mathrm{Ce}$ & 247 & 194 & 18.8 & 37.2 & 0.21 & & & \\
\hline $\operatorname{Pr}$ & & & & 5.34 & 0.02 & & & \\
\hline $\mathrm{Nd}$ & 100.9 & 74.8 & 11.5 & 24.1 & 0.1 & & & \\
\hline $\mathrm{Sm}$ & 15.2 & 12.8 & 3.49 & 6.1 & 0.02 & & & \\
\hline $\mathrm{Eu}$ & 4 & 3.22 & 1.07 & 2.06 & 0.01 & & & \\
\hline $\mathrm{Gd}$ & 10.41 & & 4.11 & 5.88 & 0.03 & & & \\
\hline $\mathrm{Tb}$ & 1.31 & 0.933 & 0.66 & 0.92 & 0.01 & & & \\
\hline Dy & & & & 5.32 & 0.02 & & & \\
\hline Ho & & & & 0.98 & 0.01 & & & \\
\hline Er & & & & 2.48 & 0.01 & & & \\
\hline $\mathrm{Tm}$ & & & & 0.31 & 0.01 & & & \\
\hline
\end{tabular}


Table 4. Cont.

\begin{tabular}{lccccc}
\hline $\mathrm{Yb}$ & 1.746 & 1.33 & 2.63 & 1.97 & 0.01 \\
$\mathrm{Lu}$ & 0.19 & 1.18 & 0.37 & 0.27 & 0.01 \\
$\mathrm{~Pb}$ & & & & 1.53 & 0.54 \\
$\mathrm{Th}$ & 12.61 & 10.61 & 1.07 & 1.29 & 0.04 \\
$\mathrm{U}$ & 2.14 & 2.06 & 0.47 & 0.41 & 0.01 \\
$\mathrm{Sc}$ & 31 & 53 & 37 & 31.4 & 0.04 \\
$\mathrm{~V}$ & 366 & 330 & & 320 & 0.21 \\
$\mathrm{Cr}$ & 253 & 610 & 196 & 280 & 0.62 \\
$\mathrm{Co}$ & 60 & 72 & 48 & 44 & 0.14 \\
$\mathrm{Ni}$ & 130 & 220 & 88 & 44.8 & 1 \\
$\mathrm{Cu}$ & 201 & 108 & 132 & 120 & 0.76 \\
$\mathrm{Zn}$ & 124 & 94 & 77 & 110 & 9.5 \\
$\mathrm{Zr}$ & 490 & 380 & 108 & 230 & 0.14 \\
$\mathrm{Nb}$ & & & & 13.6 & 0.22 \\
$\mathrm{Hf}$ & 10.51 & 8.54 & 2.46 & 5.1 & 0.09 \\
$\mathrm{Ta}$ & 8.91 & 8.24 & 0.32 & 0.91 & 0.05 \\
\hline
\end{tabular}

Note. No. 1-3, Taimyr (1-sill; 2-5, Dyumtaleysky massif); 6-22, intrusions of the Noril'sk area (6-9, Lower Talnakh, 10-13, Zelenaya Griva, 14-22, Chernogorsky); 23-28, sill-like intrusions of the Kulyumber river valley; 29-32, intrusions of the Lower Tunguska river valley (sills cutting formations: 29, Korvunchansky, 30,Degalinsky, 31, between Pelyatkinsky and Korvunchansky, 32, Nidymsky); 33-40, intrusions of the Angara river valley (33-35, Tulunsky sill, 36-40, Padunsky sill); 41-43, intrusions of the Maymecha-Kotuy area; 44, standard measurements; 45, limit of detection (LOD); N analyses: N 1-22 for samples taken from cores of boreholes (LP-5/369, title of borehole/depth in m), 23-40 for samples collected in outcrops. Data: * after [40].

The intrusions of the Lower Tunguska river valley (Figure $2 \mathrm{~b}$ ) are localized among the volcanic rocks of various formations but they also all have very similar compositions (Table 4, Figure 8). They can also be referred to the Katangsky complex based on geochemical data. A distinctive feature of these sill-like intrusions is their average magnesium content (6-7 wt \% MgO), which is very common for volcanic rocks of WPB magmatism.

Comparison of the behavior of the major components in the rocks of different intrusive complexes located within the Siberian trap province (from the key areas 1-6, Figures 8 and 9a-d) allows us to make the following conclusions. Firstly, intrusions of the Noril'sk area (Lower Talnakh, Zelenaya Griva and Chernogorsky) and Taimyr area (Dyumtaleysky massif) have a wide range of rock compositions reflecting their differentiated structure from high-Mg rock types (with $\mathrm{MgO}>25 \mathrm{wt} \%$ in troctolite, picritic gabbro-dolerite to typical gabbro and gabbro-diorites with 5-7 wt \% MgO concentrations). Intrusions from the valleys of the Kulyumber, Lower Tunguska and Angara rivers (4-6 areas) have a very consistent composition and correspond to the gabbro-dolerite. In terms of $\mathrm{SiO}_{2}$ contents, they are also close to the most acidic rock types of differentiated intrusions. On the basis of these two elements. They could be considered as differentiates of a common primary melt that formed the indicated complexes. 

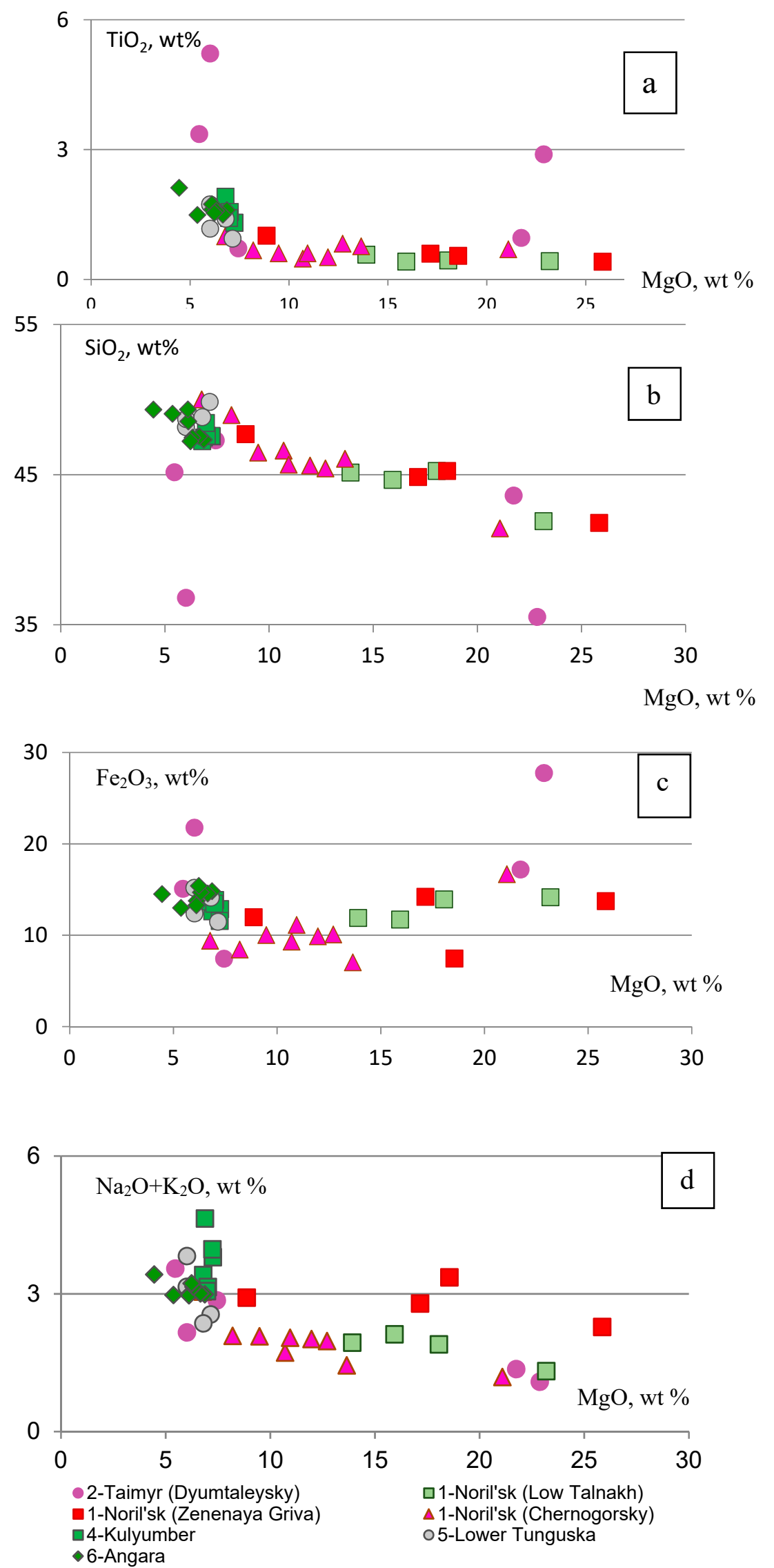

Figure 9. Diagrams of $\mathrm{MgO}-\mathrm{SiO}_{2}(\mathbf{a}), \mathrm{TiO}_{2}(\mathbf{b}), \mathrm{Fe}_{2} \mathrm{O}_{3}(\mathbf{c})$, and $\mathrm{Na}_{2} \mathrm{O}+\mathrm{K}_{2} \mathrm{O}$ (d) for volcanic rocks of the Siberian trap province. Data in Table 3. 
However, the comparison of intrusions from different areas in other elements questions this assumption. In general, the intrusions from the southern areas (4-6) of the province are richer in Fe. Ti and contain elevated concentrations of alkalis (sodium and potassium) in comparison with similar rocks from the differentiated massifs of the Noril'sk area with similar magnesium and silica contents. According to these parameters, they are close to the Dyumtalaleysky intrusion, which is characterized by elevated concentrations of $\mathrm{Fe}_{2} \mathrm{O}_{3}$ and $\mathrm{TiO}_{2}$ (and alkalis) but which has significantly greater variations of these oxides due to the varying content of titanomagnetite in the rocks (for example, analyses 1 and 3 in Table 4).

According to paleomagnetic data $[89,96]$, intrusions of both normal and reverse magnetic polarity are present in the river Lower Tunguska valley. Based on the comparison of the paleomagnetic directions, Latyshev et al. [89] suggested that the majority of normally magnetized intrusions is coeval to the above-mentioned normal polarity interval of the volcanic section (comprising the uppermost Korvunchansky, Nidymsky, and Kochechumsky formations) and, consequently, to the main stage of the "trap" or "platform" magmatic activity in the Noril'sk and Lower Tunguska region. As to the intrusions of reverse polarity, some cut the normally magnetized volcanic section and should be younger than the volcanic sections of the Noril'sk and Tunguska regions. Thus, the emplacement of intrusions referred to as the Katangsky complex was rather continuous and lasted during the whole main stage of trap magmatic activity and after it.

The southeast part of the province (the Angara-Taseeva syncline) is characterized by the presence of large intrusive bodies (sills). Some are hundreds of kilometers in length and their thickness reaches up to $400 \mathrm{~m}$. Nevertheless, they are weakly differentiated or undifferentiated and composed of ordinary gabbro that has a similar composition to the intrusions of the Katangsky complex (Figure 8). Three large sills were analyzed: Padunsky, Tulunsky, and Nizhneudinsky (Table 4, samples 1.1T, 1.2T, 1.3T). The latter has some differences compared with the first two massifs: their rare element patterns show steeper slopes and smaller Ta-Nb anomalies. According to paleomagnetic data [83,88], all of these sills have reverse polarity. Since the major part of volcanic sequences of the Noril'sk and Lower Tunguska regions demonstrated normal polarity, the Angara-Taseeva intrusions cannot be coeval with them. Based on paleomagnetic and geochronological data, Latyshev et al. [89] suggested that the emplacement of the Padunsky sill took place just after the termination of the main stage of the volcanic activity in the Siberian platform. However, the accurate correlation of the Padunsky sill and other intrusions to the volcanic section requires additional investigation.

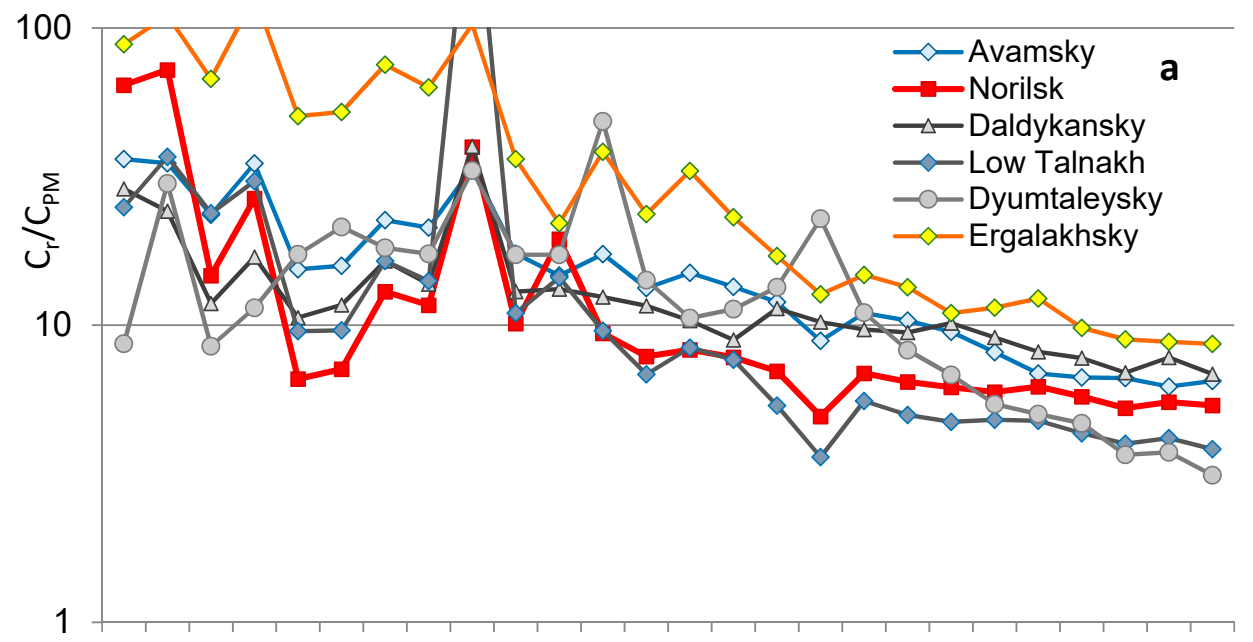

$\mathrm{Rb} \mathrm{Ba}$ Th $\mathrm{U}$ Nb Ta La Ce Pb Pr Sr Nd Sm Zr Hf Eu Ti Gd Tb Dy Ho Y Er Yb Tm Lu

Figure 10. Cont. 

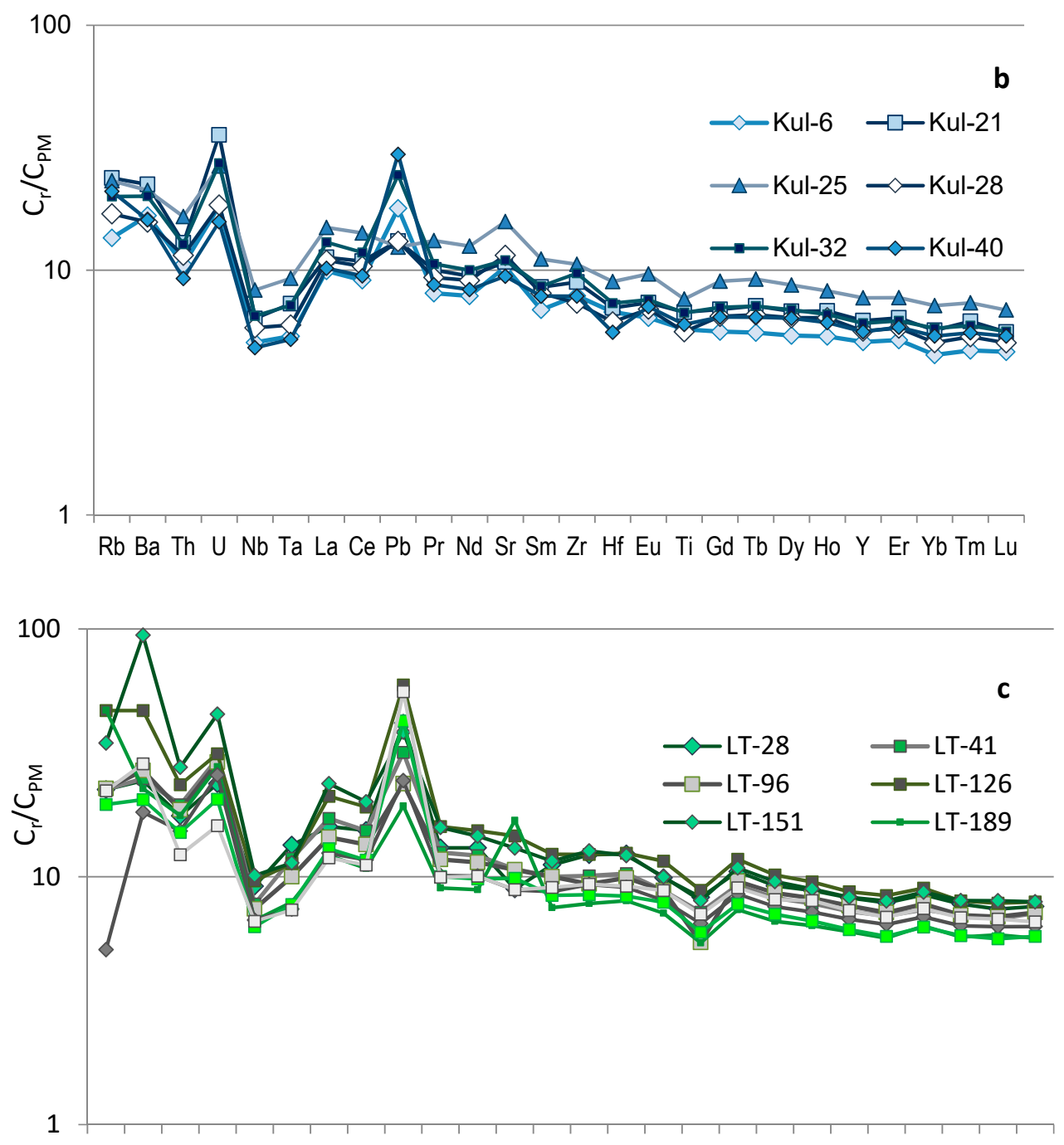

Rb Ba Th U Nb Ta La Ce Pb Pr Nd Sr Sm Zr Hf Eu Ti Gd Tb Dy Ho Y Er YbTm Lu

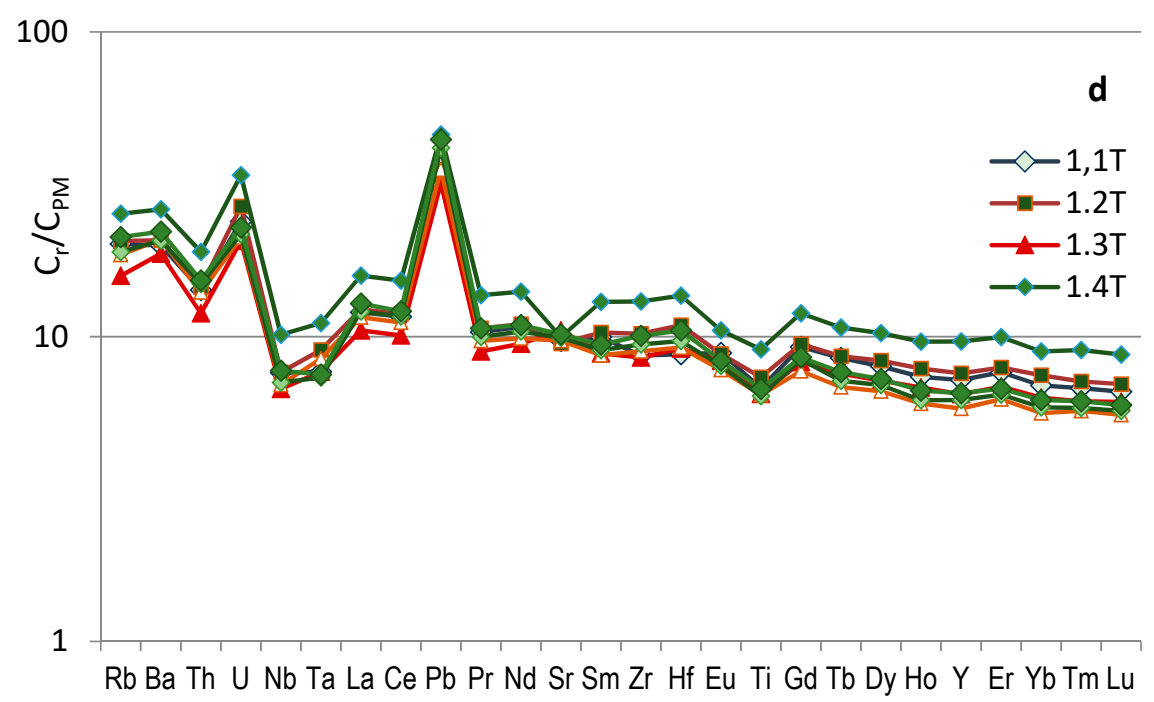

Figure 10. Representative spider diagrams of the intrusive rocks of the Siberian trap province: (a) Noril'sk area; (b) Kulyumber river valley; (c) Lower Tunguska river valley; (d) Angara river valley. Data: (a) after [14]; (b-d) in Table 4. 


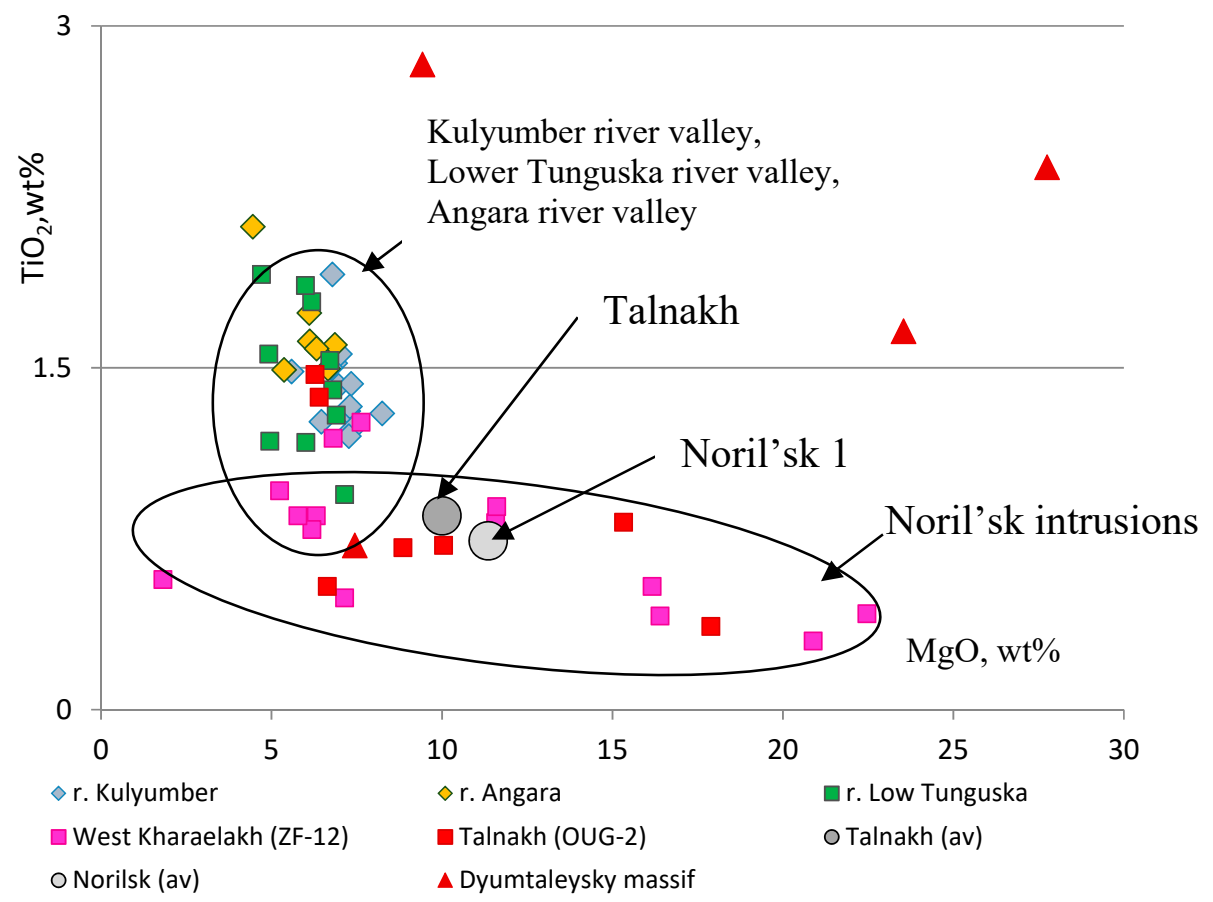

Figure 11. Diagram of $\mathrm{TiO}_{2}-\mathrm{MgO}$ for intrusive rocks of the Siberian province. Average mean compositions of the Talnakh intrusion based on 29 boreholes, the Noril'sk1 intrusion on 54 boreholes [4].

The intrusive rocks' diversity is a specific feature of the northern areas of the Siberian trap province, the Norilsk-Taimyr and Maymecha-Kotuy areas [14,34,45,70]. The intrusions of these regions demonstrate the great variety in $\mathrm{MgO}$ and $\mathrm{TiO}_{2}$ contents (Figure 11) and are typical of the effusive rocks. As for the Angara-Taseeva syncline and the Tunguska syneclise, the majority of intrusions and volcanic rocks in these regions regardless of their age demonstrate the uniform geochemical features similar to lavas of the upper formations from the Norilsk region.

\section{Tectonic Position of Intrusions within the Siberian Trap Province}

The discovery of PGE-Cu-Ni deposits in the Noril'sk and in Taimyr regions stimulated the interest in the tectonic structure of these regions [97,98]. This area, including the Yenisey-Khatanga trough and adjacent structures (Figure 1a), fundamentally differs from the rest of the Siberian platform: firstly, there is a low-viscosity zone at a depth of around $80 \mathrm{~km}$ as was mentioned earlier (Figure 1c). Secondly, the Archaean-Proterozoic crystalline basement is covered here by deep volcanic-sedimentary deposits ranging in age from Ediacaran to Lower Paleozoic in contrast to the thin cover of the Siberian platform [18]. Some of these sediments occur in rift zones (Figure 12) characterized by specific magmatism and mineralization. Geopotential field tomography data demonstrate the existence of a large fault zone which penetrates the upper mantle and extends into the Earth's crust [22]. The dislocation has the shape of a flattening of a funnel extended in the north-south direction with a submerging channel in the upper mantle located in the Igarka area (Figure 1c).

Malich et al. [29] traced the Igarka-Noril'sk paleo-rift (Figure 1b) from the Igarka district to the Ikens trough. Then it was traced below the Yenisey-Khatanga trough in the north and finally into the the Taimyr peninsula [19]. The eastern border of the paleo-rift is the Lamsko-Letninsky fault and its north boundary is the Yenisey fault, which bounds the West Siberian plate and the north border is the Yenisey-Katanga fault. The Igarka-Noril'sk paleo-rift formed in the Proterozoic was reactivated in the Devonian and was most active in the Triassic. Geophysical data demonstrate the complex structure of this ancient rift that had increased mobility and permeability and was the focus of intensive magmatism [19] which produced a high diversity of rocks (alkaline, subalkaline, ultrabasic, 
and basic). These magmas formed numerous lava flows and intrusions differing in morphology and composition (the Noril'sk-Taimyr and Maymecha-Kotuy areas).

The Noril'sk-Igarka paleo-rift (Figure 12) is an intercontinental rift formed during the divergent stage of lithospheric development; it is associated with horizontal extension, thinning and breakup of the lithosphere. It is characterized by a high density of faults and a large volume of intruded mantle material in the lower crust (the total volume of igneous rocks within the Noril'sk-Kharaelakh trough is estimated at 50 to 70 thousand $\mathrm{km}^{3}$, thus indicating an extremely high degree of permeability of this structure $[8,18,99]$.

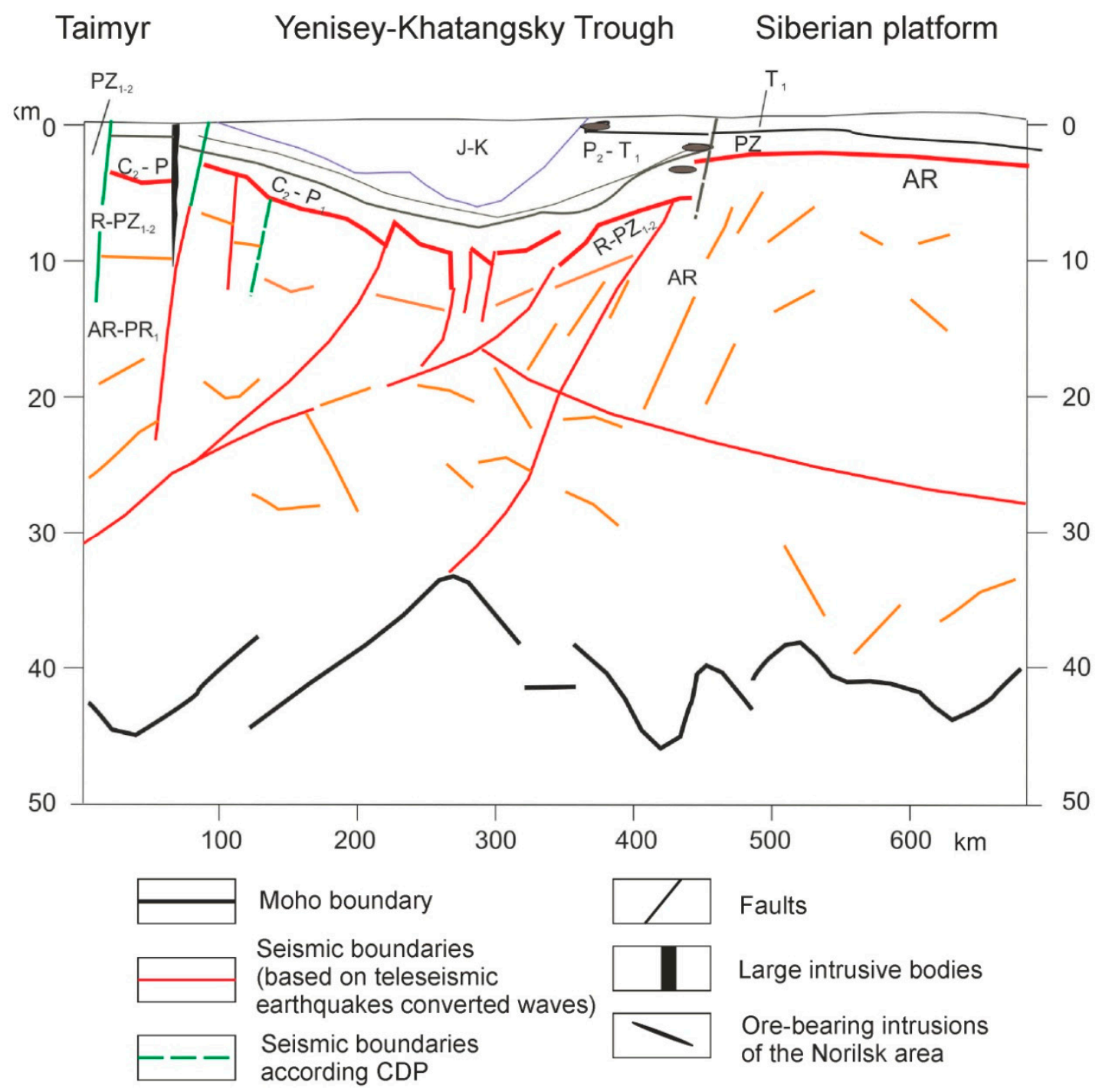

Figure 12. Structure of Yenisey-Khatanga rift zone (based on seismic data of Kostuchenko [21], with changes).

Based on the gravimetric and magnetic survey data (1:200,000 scale), the Noril'sk-Igarka paleo-rift is characterized by a zone of a gravity and magnetic high fields that is approximately $100-200 \mathrm{~km}$ wide $[20,99]$. It coincides with the northwest margin of the Siberian platform. Of special interest are three east-west branches of the paleo-rift that have similar physical properties. These deep structures are clearly seen on the maps of various geophysical transformations, in particular, the first vertical derivative of the geomagnetic field calculated at an altitude of $20 \mathrm{~km}$ (Figure 13a) using the source-approximation algorithm [100]. The contrasting isolation of deep structural features occurs due to the suppression of anomalies of near-surface objects when recalculating in the upper 
half-space in combination with the "focusing" of a useful signal due to differential transformations. The northernmost branch of the paleo-rift is the Bolsheavamsky. In its limits, according to the morphological features of the fields, a sublatitudinal disjunctive dislocation traverses the territory approximately at the latitude of the Talnakh deposit. The Dyupkunsky branch (Figure 13a, number 2), which covers the basins of the Gorbiachin, Kulyumber, and Kureyka rivers (Figure 1b), is close to the Noril'sk-Igarka rift. To the south of this area, at the latitude of Turukhansk village (Figure 13a, number 3), one can observe the presence of the third Lower Tunguska branch, which is less contrasted in geophysical fields.

A clearer picture occurs on the map of the iso-anomalies of the pseudo-gravitational field (Figure 13b) [101,102]. Maps of the pseudo-gravitational field provide a clearer picture of the geological structure of an area than magnetic field maps due to the elimination of the effect of superimposing positive and negative neighboring anomalies. These regional structures are interpreted to control the location of ore-bearing intrusions of the Noril'sk area (Bolsheavamsky), the Kulyumber river (Siluriyskaya, Dyupkun), and the Kureyka river (large Djaltulsky massif with PGE-Cu-Ni and native Fe mineralization) $[19,103]$. We suggest that the Lower Tunguska branch (number 3 in Figure 13b) is similarity prospective for PGE-Cu-Ni deposits.
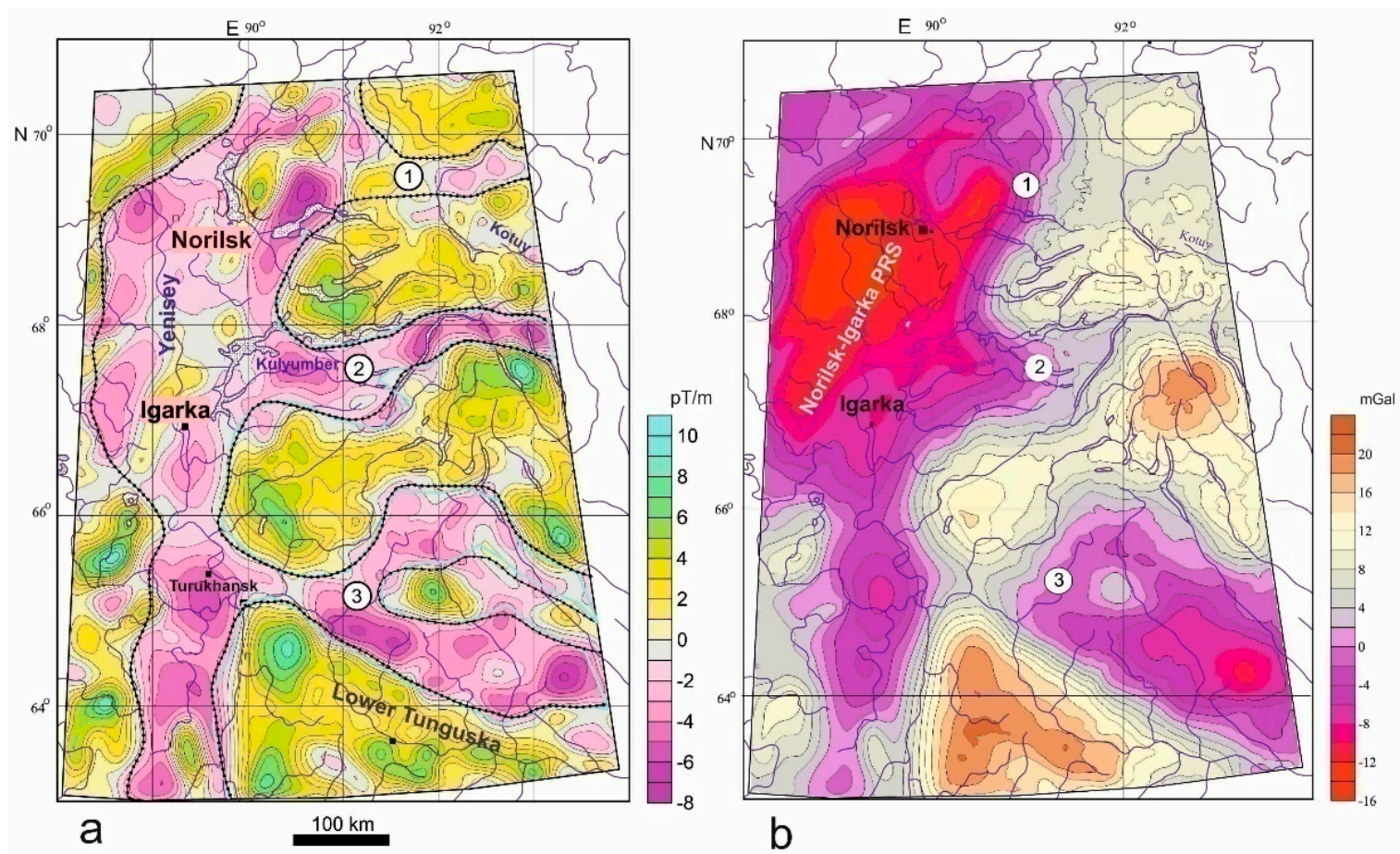

Figure 13. The Noril'sk-Igarka paleorift on maps of the first vertical derivative of the anomalous magnetic field at an altitude of $20 \mathrm{~km}$ (a) and iso-anomalous of pseudo-gravitation field (b). The numbers refer to the branch of the paleo-rift system (PRS): 1, Bolsheavamsky; 2, Dyupkunsky; and 3, Lower Tunguska.

\section{Discussion}

\subsection{Analysis of the Noril'sk Deposits Models}

The origin of the PGE-Cu-Ni Noril'sk ores has been under examination since the discovery of the Talnakh and Oktyabr'skoe deposit in the 1960s. The unusually high concentration of sulfide minerals combined with their very high PGE tenors posed a question about the relationship between ore-forming processes and the magmatism that produced the Siberian traps province.

In recent years, the Noril'sk deposits have been linked directly to the Siberian superplume [104-106]. Kuzmin and Yarmolyuk [107] suggested that the Siberian platform and the Mongolia-Okhotsk belt (located to the south of the Siberian platform) comprise a vast plume province 
where deposits have a zonal distribution in relation to the center in the Putorana plateau (Figure 1b). The position of the center of the plume was considered to be in the location of high-Mg and alkaline rocks in the northern Putorana plateau. Dobretsov et al. [105] suggested that plume rocks enriched in Pt and Pd led to the formation of the Noril'sk deposits. However, the PGE contents in the Siberian traps in the Noril'sk area has been documented by many geologists [108-111], who demonstrated that their PGE contents (including those formed by mantle-derived magmas, such as the Gudchikhinsky basalt and picrite) are similar to those in other flood-basalt provinces worldwide [112,113]. Begg et al. [114] in the "craton margins" model suggested the formation of the majority of deposits at the boundaries of ancient lithospheric plates. These deposits' positions are explained by a possibility of easier emplacement of a large volume of plume magmas into permeable zones at the contacts of rigid structures. However, in the case of the Noril'sk deposits, simultaneously with intrusions emplacement on the border of the Siberian platform and Taimyr, the huge volume of magmas penetrated in the center of the Siberian platform (the plateau Putorana, the Tunguska syncline) formed a thick series of the volcanic rocks of the Morongovsky and Mokulaevsky formations (their synchronism is confirmed by paleomagnetic data [85]). In addition, the deposits of the same age (early Mesozoic) on the south contact of the platform with the Mongol-Okhotsk belt are unknown. The $\mathrm{Cu}-\mathrm{Ni}$ deposits of this area shown by the authors in Figure 1 [114] are Proterozoic and do not relate to the Siberian trap province. Other trap provinces also lack large deposits. Furthermore, the authors of this model explain the absence of major deposits that should be formed directly on the margins of large oceans or at convergent margins by possible loss of sulfides rather than a genetic limitation. In contrast, we believe that a genetic limitation occurred (see below).

Thus, there must be mechanisms for concentration of metals in ore. They have been suggested by geologists in numerous models. As agents of concentrators, both magmas and fluids have been considered $[5,8,44,115]$. The most widespread hypothesis is a magmatic origin of the Noril'sk deposits based on evidence of ore minerals crystallization from high-temperature sulfide melt and low-water solubility in basaltic melts. There are two contrasting groups of models explaining magmatic genesis of ore either in a close magmatic system or in an open one. The latter is very popular at present. Rad'ko [10] supposed that the Noril'sk ores were formed in an open magmatic system where ore-bearing intrusions were parts of a common system with lavas. The sulfides settled in a horizontal section of the channels due to the decrease in the rate of magma flow when it leaves a vertical channel. Naldrett [11] further developed this model and linked the intrusions with the volcanic rocks of the Lower Nadezhdinsky formation that are strongly depleted in Cu and Ni [100,101]. Brügmann et al. [108] and Lightfoot and Keays [109] suggested that the metal depletion had occurred in a deep seated magma chamber due to assimilation of crustal rocks by mantle-derived magmas; this resulted in the ore metals being deposited at depth and later being picked up and transported to the sills by the magmas that formed the Morongovsky formation. Great importance was attached to the assimilation of anhydrite as it has heavy $S$ isotopic compositions similar to the $S$ isotopic composition of the ore $[12,116,117]$. The main advantage of this model is the effective mechanism of metal concentration from a large volume of melt flowing to the surface. However, it does not take into account many important facts. First of all, there is no evidence of geological links between the lavas and intrusions [14,118,119]. Secondly, it does not explain the discrepancy between the composition of the intrusions and lavas ( $\mathrm{MgO}$ of $10-12 \mathrm{wt} \%$ and $\mathrm{TiO}_{2}$ of $0.8 \mathrm{wt} \% \delta^{34} \mathrm{~S}=+12 \%$ for intrusions, compared with the Morongovsky basalt with $6-8 \mathrm{wt} \% \mathrm{MgO}, \mathrm{TiO}_{2}=1.3 \mathrm{wt} \%$ and $\delta^{34} \mathrm{~S}=<5 \%$ as well as other isotopic data. Secondly, it also does not take into account the restricted scale of assimilation in the intrusions [120]. Heavy sulfur isotopes were found in the rocks that were formed from the most primitive mantle magmas in the north part of the Siberian province (in the Gudchikhinsky Formation [121] and in the Dyumtaleysky massif $[14,16,67]$. Thus, $\delta^{34} S=+12 \%$ is a primary feature of the mantle in this area in the Early Mesozoic (251 Ma). Thirdly, this model does not pay attention to the position of ore-bearing intrusions within the Siberian trap province. According to the hypothesis 
of ore formation in an open system, we should expect the discovery of ore deposits in many places within the Siberian province where they are not found.

The second model of the deposits origin suggests that ores were formed in a closed magmatic system. Although many geologists $[4,7-9,14,43,68,118,119]$ advocated a closed magmatic system, the greatest contribution to this model was made by Likhachev $[5-7,45]$. This hypothesis supposes a differentiation of primary mantle magma enriched in sulfides before its emplacement in the modern chamber. Sulfides were brought there with cumulus minerals (olivine and plagioclase). Based on this idea, ore-bearing intrusions are results of separate magma impulses, and their internal structure has a good correlation with this model. It is important to emphasize that the position of ore-bearing intrusions in the north of the province was explained by the presence of deep faults along which mantle-derived magma ascended [7]. The Noril'sk-Kharaelakh zone was a suitable structure for the penetration of mantle magma into the crust.

But recent geological and geochemical data revealed some shortcomings of this model. For example, geochemical data demonstrate that the ore-bearing intrusions are not the products of primitive mantle magmas. They are characterized by crustal isotopic compositions with ${ }^{86} \mathrm{Sr} /{ }^{86} \mathrm{Sr}$ ranging from 0.705 to 0.709 and $\varepsilon_{\mathrm{Nd}}$ from -1 to $+2[26,27,69,70]$. To explain these data it was suggested that the primary magmas were contaminated by $30 \%$ of crustal material $[26,37,38]$ due to anhydrite assimilation [120]. This is too large a volume of foreign material for ultrabasic-basic magmas, which are characterized by a limited ability of assimilation as shown for the Noril'sk intrusions [120] and for many other plutonic bodies.

Another imperfection of this model is shown by the interpretation of geophysical methods. This interpretation indicates that faults controlling the location of ore-bearing intrusions are not traced deeper than 20-30 km [22,102]. At the same time, mantle-derived faults were also found in the central parts of the Siberian platform and not only in its marginal part [19]. Thus, the location of the deposits in the Noril'sk and Taimyr areas does not depend only on deep faults occurrence, and their concentration in the north of the province requires a special interpretation.

\subsection{Summary of the Results and Origin of the Noril'sk}

Data of this article demonstrate that the PGE-Cu-Ni deposits were found only in the north part of the province (the Noril'sk, Taimyr, and Maymecha-Kotuy areas) of the province which differs from the remaining areas (the Kulyumber, Lower Tunguska and Angara river valleys) in a high compositional diversity of magmatic rocks. Indeed, the Kulyumber river area shows many intermediate features between the north and south territories due to the presence of intrusive bodies close in composition both to the Noril'sk area and to the Tunguska syncline. Based on significant predomination of the Katangsky complex intrusions in the Kulumber river valley we attributed this area to the south part of the province although this decision is provisional.

Table 5 summarizes data on magmatic rocks. It is obvious that all geological, petrological, and geochemical parameters of the north and south of the Siberian trap province have many differences. First of all, high-magnesium volcanic rocks (picrite, picritic basalt, maymechite) and alkalis rocks (trachyandesite, basaltic trachyandesite, trachybasalt) having high variations of $\mathrm{MgO}(3.6-37.2 \mathrm{wt} \%)$ and alkalis (0.9-10.4 wt \%) are widespread only in the north. Rocks of normal alkalinity $(<5 \mathrm{wt} \%$ $\mathrm{Na}_{2} \mathrm{O}+\mathrm{K}_{2} \mathrm{O}$ ) and with medium magnesium oxide contents $(6-7 \mathrm{wt} \% \mathrm{MgO})$ dominate in the Tunguska syncline, while they also occur in the Noril'sk area and Taimyr.

Large layered differentiated intrusions (including ore-bearing massifs) are located only in the north, northwest Siberian platform and south Taimyr peninsula. Due to the presence of these intrusions the composition of intrusive rocks ranges from melatroctolites to leucogabbro (Figure $8 \mathrm{a}, \mathrm{b}$ ) and gabbro-diorites. Their composition changes from ultrabasic to basic $\left(\mathrm{SiO}_{2}\right.$ varies from 42 to $50 \mathrm{wt} \%$, samples with $35 \% \mathrm{SiO}_{2}$ are enriched in titanomagnetite, Table 4) where $\mathrm{MgO}$ reaches $26 \mathrm{wt} \%$. We do not regard here the Guli alkaline-ultramafic massif because it needs a special interpretation. Intrusions of the Tunguska syncline and its framework are characterized by very stable composition corresponding 
to tholeitic basalt $\left(6-7 \mathrm{wt} \% \mathrm{MgO}, 1-1.7 \mathrm{wt} \% \mathrm{TiO}_{2}\right.$ on average and elevated $\mathrm{Na}_{2} \mathrm{O}+\mathrm{K}_{2} \mathrm{O}$ ranging from 2.9 to $4.6 \mathrm{wt} \%$ ) (Table 5).

Table 5. Comparison of the north and south of the Siberian trap province.

\begin{tabular}{|c|c|c|}
\hline Characteristics & $\begin{array}{c}\text { North } \\
\text { (Noril'sk, Taimyr, } \\
\text { Maymecha-Kotuy) }\end{array}$ & $\begin{array}{c}\text { South } \\
\text { (Kulyumber river valley, } \\
\text { Tunguska syncline, Angara } \\
\text { river valley) }\end{array}$ \\
\hline \multicolumn{3}{|c|}{ Volcanic rocks } \\
\hline Types of rocks & $\begin{array}{l}\text { Picrite, maymechite, andesite, } \\
\text { trachyandesite, tholeiitic basalt }\end{array}$ & Tholeiitic basalt, andesite \\
\hline \multirow{4}{*}{$\begin{array}{l}\text { Geochemistry of the volcanic rocks: } \\
\text { major components variations, wt \% }\end{array}$} & $\mathrm{MgO}=3.6-37.2$ & $\mathrm{MgO}=5.6-7.2$ \\
\hline & $\mathrm{TiO}_{2}=0.8-3.9$ & $\mathrm{TiO}_{2}=1.0-1.6$ \\
\hline & $\mathrm{Na}_{2} \mathrm{O}+\mathrm{K}_{2} \mathrm{O}=0.9-10.4(6.2)$ & $\mathrm{Na}_{2} \mathrm{O}+\mathrm{K}_{2} \mathrm{O}=2.2-4.3$ \\
\hline & $\mathrm{La} / \mathrm{Sm}=2.0-10.4$ & $\mathrm{La} / \mathrm{Sm}=2.0-3.5$ \\
\hline \multirow{2}{*}{ Rare elements ratios } & $\mathrm{Gd} / \mathrm{Yb}=1.4-6.3$ & $\mathrm{Gd} / \mathrm{Yb}=1.4-1.6$ \\
\hline & $\begin{array}{l}\mathrm{U} / \mathrm{Nb}=0.02-0.2 \\
\text { Intrusive rocks }\end{array}$ & $\mathrm{U} / \mathrm{Nb}=0.06-0.1$ \\
\hline Morphology of intrusive bodies & $\begin{array}{c}\text { Large plutons, chonolites, sills, } \\
\text { dikes }\end{array}$ & Sills \\
\hline Thickness, $\mathrm{m}$ & $5-600$ & $10-400$ \\
\hline \multirow{5}{*}{ Geochemistry, major elements, wt \% } & $\begin{array}{l}\text { Strong: Troctolite-olivine } \\
\text { gabbro-gabbro-diorite }\end{array}$ & Week: olivine gabbro-gabbro \\
\hline & $\mathrm{MgO}=3.6-37.2$ & $\mathrm{MgO}=4.5-7.3$ \\
\hline & $\mathrm{TiO}_{2}=0.8-3.8$ & $\mathrm{TiO}_{2}=1.17-2.74$ \\
\hline & $\mathrm{Na}_{2} \mathrm{O}+\mathrm{K}_{2} \mathrm{O}=1.1-3.9$ & $\mathrm{Na}_{2} \mathrm{O}+\mathrm{K}_{2} \mathrm{O}=2.9-4.6$ \\
\hline & $\mathrm{La} / \mathrm{Sm}=1.5-8.3$ & $\mathrm{La} / \mathrm{Sm}=1.9-2.9$ \\
\hline \multirow{2}{*}{ Rare elements ratios } & $\mathrm{Gd} / \mathrm{Yb}=1.6-6.0$ & $\mathrm{Gd} / \mathrm{Yb}=1.4-1.7$ \\
\hline & $\mathrm{U} / \mathrm{Nb}=0.02-0.3$ & $\mathrm{U} / \mathrm{Nb}=0.05-0.3$ \\
\hline \multirow[t]{2}{*}{ Mineralization } & $\begin{array}{l}\text { Rich massive ore - sulfide, } \\
\text { titanomagnetite + poor ore }+ \\
\text { barren intrusions }(60 \%)\end{array}$ & $\begin{array}{l}\text { Poor mineralized }+ \text { barren } \\
\quad \text { intrusions }(99 \%)\end{array}$ \\
\hline & Structure & \\
\hline \multirow{3}{*}{$\begin{array}{c}\text { Type } \\
\text { Depth of Mokho, } \mathrm{km} \\
\text { Low velocity }(8.1 \mathrm{~m} / \mathrm{s}) \text { zone at the } \\
\text { depth, } \mathrm{km}\end{array}$} & Rift & Platform \\
\hline & $31-32$ & $47-51$ \\
\hline & 80-100 & - \\
\hline
\end{tabular}

Based on the data given in this article, we believe that the model of ore deposit origin in a closed magmatic system is better supported than ore origin in an open system. We interpret the origin of the uniquely large and high PGE tenor Noril'sk deposits to be the result of the melting of protolithes that had already been enriched in sulfides during a number of processes (Figure 14), including (i) their initial crystallization in Proterozoic magma, (ii) their possible concentration during metamorphism of the rocks in the long-lived rift structures (e.g., the Igarka-Noril'sk and Yenisey-Khatanga troughs) during pre-Mesozoic time, and (iii) their mobilization and transport by the trap magmas ( \pm additional sulfides) in the Early Mesozoic. The possibility of entraining (up to 10\%) sulfide melt in the form of small droplets has been experimentally demonstrated by Likhachev [7]. 


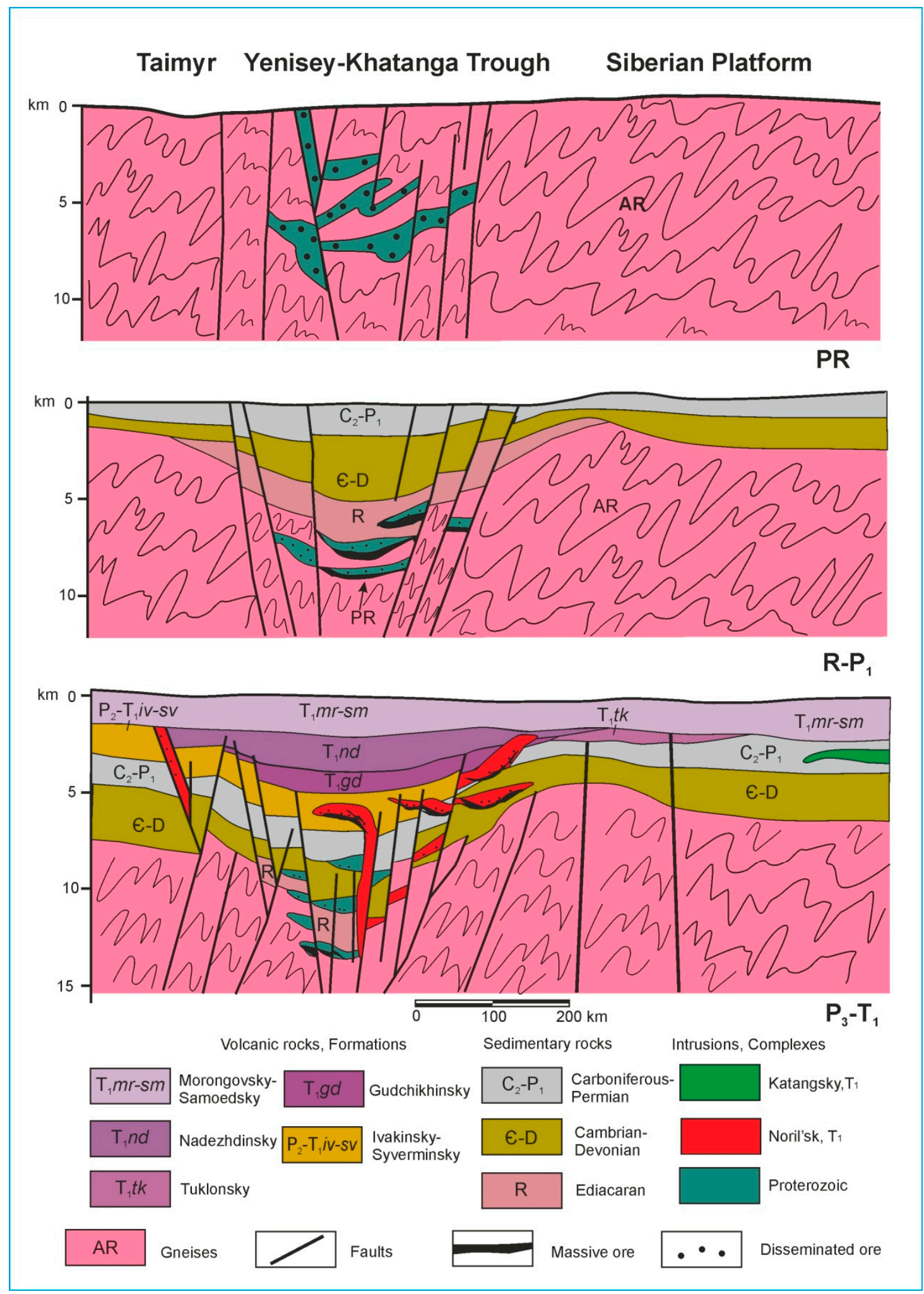

Figure 14. The main stages of the deposits formation (PR, R-P,$P_{3}-T_{1}$ ). Ore-bearing intrusions (the Noril'sk Complex) are shown out of scale.

We propose the following scenario of the formation of the deposits in the north part of the Siberian trap province. Based on the occurrences of the PGE-Cu-Ni Proterozoic deposits in the eroded south part of the Siberian platform (Chineysky, Yoko-Dovyren, Chayskoe, Nirungdinsky; 1880-740 Ma) it is 
suggested that similar deposits could exist in the north part of the Siberian province as well. It is a long-lived area which underwent numerous geological processes. The ores of small, middle or large Proterozoic deposits could have been combined into a single deposit during metamorphic processes (that took place from 1880 to $251 \mathrm{Ma}$ ). As a result, very large concentrations of metals could be formed. During the next stage the sulfides from the lower crust (that formed the Noril'sk deposits) and the mantle (the Dyumtaleysky massif) were transported by trap magmas (that may have contributed additional metals) to the current position. Thus, the sulfides passed a long way from their generation to settling interacting with a large magmas volume during pre-trap and trap per se stages. A major reason for high PGE tenors of the Noril'sk ore may have been the interaction of the pre-existing sulfides with the large volume of the trap magma. The other $\mathrm{Cu}-\mathrm{Ni}$ deposits had no the second stage in their origin, their magmas did not ascend from the crystal basement to the upper horizons of the platform and sulfides did not pass a long way. This is also the main reason for the lack of $\mathrm{Cu}-\mathrm{Ni}$ deposits in the ocean margins: they do not have a long geological history that is needed for sulfide accumulation.

Another possible reason for the high-PGE tenor of the Noril'sk ore is the enrichment of magmas by crustal sediments with high platinum contents (similar to the Sukhoi Log deposit in the south of the Siberian platform). It is important to emphasize the stochastic nature of sulfide accumulation in magmatic processes. The irregular distribution of sulfides in the intrusions of the Noril'sk area (from extra-large deposits to barren massifs) confirms this conclusion. Thus, the main idea of this model is that the formation of extra-large sulfide orebodies is not a one-step process. In contrary, we can get only poor mineralization.

\section{Conclusions}

1. The PGE-Cu-Ni deposits are constrained to a narrow corridor within the Arctic areas of the Siberian trap province that are characterized by the maximal geochemical diversity of the volcanic and intrusive rocks. The deposits are related to the high-Mg magmas (10-12 wt \% MgO) with crustal (the Noril'sk deposits) and mantle (the Dyumtaleysky deposit) characteristics.

2. The zones of maximal multiplicity of igneous rocks differ from platform areas, and represent paleo-rifts with specific structure. They have a long-term geological history which stimulated sulfide accumulation during several stages from the Proterozoic to the Mesozoic.

3. The most important areas for prospecting for new deposits inside the Siberian platform are the Dyupkun and Lower Tunguska branches of the Noril'sk-Igarka paleo-rift.

4. We cannot expect the discovery of world-class deposits in any parts of trap provinces because the accumulation of sulfides occurs as a result of a number of geological processes that took place over a long period of time.

Supplementary Materials: The following are available online at http:/ /www.mdpi.com/2075-163X/9/1/66/s1, Table S1: List of the sudied samples.

Author Contributions: N.A.K. studied rocks on field trips and wrote the text, A.V.L. sampled intrusions in the Tunguska and Angara valleys and gave interpretation of paleomagnetic data. A.S.D. got geophysical data and interpreted them, B.I.G. carried out sampling of intrusions in the Noril'sk and Kulyumber areas. E.M.M. and A.A.M. collected samples in Taimyr, N.M.S. prepared samples for analytical study. Y.V.B. analyzed rare elements in rocks by ICP-MS, A.I.Y. did XRF analyses. A.M.A. carried out petrochemical calculations.

Funding: This study was supported by the Russian Foundation for Basic Research (projects nos. 16-35-60114. 17-05-01121; 18-05-70094: 18-35-20058.).

Acknowledgments: The authors thank the geologists of Ltd. Noril'skgeologia A. Lapkovsky, I. Sidorenko and the students of Lomonosov Moscow State University M. Nesterenko, D. Korshunov and E. Ostrovsky (Tomsk State University) for their assistance in the field trip work. We are very grateful the reviewers for their helpful comments and recommendations.

Conflicts of Interest: The authors declare no conflict of interest. 


\section{References}

1. Kamo, S.L.; Czamanske, G.K.; Amelin, Y. Rapid eruption of Siberian flood-volcanic rocks and evidence for coincidence with the Permian-Triassic boundary and mass extinction at 251 Ma. Earth Plan. Sci. Lett. 2003, 214, 75-91. [CrossRef]

2. Burgess, S.D.; Bowring, S.A. High-precision geochronology confirms voluminous magmatism before. during. and after Earth's most severe extinction. Sci. Adv. 2015, 1, e1500470. [CrossRef] [PubMed]

3. Kotulsky, V.K. About Effusive Rocks of the Noril'sk Region. Noril'sk Bull. Sci.-Tech. Inf. 1943, 2, 28-36. (In Russian)

4. Godlevsky, M.N. Traps and Ore-Bearing Intrusions of the Noril'lsk Region; Gosgeoltekhizdat: Moscow, Russia, 1959; p. 61. (In Russian)

5. Likhachev, A.P. The Role of Leucocratic gabbro in the origin of Noril'sk differentiated Intrusions. Izv. Akad. Nauk SSSR. Ser. Geol. 1965, 12, 50-66. (In Russian)

6. Likhachev, A.P. Trap Magmatism and Platinum-Copper-Nickel Ore Mineralization in the Noril'sk District. Otech. Geol. 1997, 10, 8-19. (In Russian)

7. Likhachev, A.P. Platinum-Copper-Nickel and Platinum Deposits; Eslan: Moscow, Russia, 2006; p. 496. (In Russian)

8. Dyuzhikov, O.A.; Distler, V.V.; Strunin, B.M.; Mkrtychyan, A.K.; Sherman, M.L.; Sluzhenikin, S.F.; Lurye, A.M. Geology and Ore Potential of the Noril'sk Ore District; Nauka: Moscow, Russia, 1988; p. 238. (In Russian)

9. Ryabov, V.V.; Shevko, A.Y.; Gora, M.P. Trap Magmatism and Ore Formation in the Siberian Noril'sk Region; Springer: Amsterdam, The Netherlands, 2014.

10. Rad'ko, V.A. Model of the Dynamic Differentiation of Intrusive Traps from the Northwestern Siberian Platform. Geol. Geophys. 1991, 11, 19-27. (In Russian)

11. Naldrett, A.J. A model for the Ni-Cu-PGE Ores of the Noril'sk Region and Its Application to Other Areas of Flood Basalts. Econ. Geol. 1992, 87, 1945-1962. [CrossRef]

12. Li, C.S.; Ripley, E.M.; Naldrett, A.J. A new genetic model for the giant Ni-Cu-PGE sulfide deposits associated with the Siberian flood basalts. Econ. Geol. 2009, 104, 291-301. [CrossRef]

13. Barnes, S.J.; Mungall, J.E.; Le Vaillant, M.; Godel, B.; Lesher, C.M.; Holwell, D.A.; Lightfoot, P.C.; Krivolutskaya, N.A.; Wei, B. Sulfide-silicate textures in magmatic Ni-Cu-PGE sulfide ore deposits. Disseminated and net-textured ores. Am. Miner. 2017, 102, 473-506. [CrossRef]

14. Krivolutskaya, N.A. Siberian Traps and Pt-Cu-Ni Deposits in the Noril'sk Area; Springer: Amsterdam, The Netherlands, 2016; p. 361.

15. Tesakov, Y.I. The Silurian Basin of Eastern Siberia: Basin Paleogeography (on a Chronosonal and Biogeocenotic Basis); INGG: Novosibirsk, Russia, 2014; Volume 3, p. 389. (In Russian)

16. Krivolutskaya, N.A. Mantle origin of heavy isotopes of sulfur in ores of the Noril'sk deposits: Dokl. Earth Sci. 2014, 454, 76-78. [CrossRef]

17. Staroseltsev, V.S. Tectonic of Lava Plateau; Nedra: Moscow, Russia, 1989; p. 258. (In Russian)

18. Rempel, G.G.; Parshukov, N.P.; Vayvod, E.A. Volumetric modeling of the tuffaceous-effusive strata of the Noril'sk region according to aeromagnetic survey data and the prospecting of copper-nickel mineralization. Rus. Geol. Geophys. 1990, 7, 87-98. (In Russian)

19. Orlov, V.P.; Malitch, N.S. (Eds.) Eastern Siberia. Geology and Deposits of Russia; VSEGEI: S-Petersburg, Russia, 2002; p. 396. (In Russian)

20. Dolgal, A.S.; Kalinin, D.F.; Oleshkevich, O.I.; Siminov, O.N. Application of computer technologies for interpreting geopotential fields in prospecting of platinum-copper-nickel mineralization. Razvedka Okhrana Nedr 2006, 8, 57-65. (In Russian)

21. Kostuchenko, S.L. Structure of the crust and surface mechanisms of formation of near-Arctic continental sedimentary basins in Siberia. Reg. Geol. Metall. 2000, 10, 125-135. (In Russian)

22. Pavlenkova, N.I.; Pavlenkova, G.A. Structure of the Crust and upper Mantle of the Northern Eurasia Based on Data of Seismic Profiles with Nuclear Explosion; Geokart. GEOS: Moscow, Russia, 2014; p. 191. (In Russian)

23. Rudnic, R.L.; Gao, S. Composition of the continental crust. In Treatise on Geochemistry; Elsivier-Pergamon: Oxford, UK, 2003; pp. 1-64. 
24. Sharma, M.; Basu, A.R.; Nesterenko, G.V. Temporal Sr-, Nd-, and Pb-Iisotopic variations in the Siberian flood basalts: Implications for the plume source characteristics. Earth Planet. Sci. Lett. 1992, 113, 365-381. [CrossRef]

25. Nesterenko, G.V.; Al'mukhamedov, A.I. Geochemistry of Differentiated Traps; Nauka: Moscow, Russia, 1973; p. 128. (In Russian)

26. Wooden, J.L.; Czamanske, G.K.; Bouse, R.M.; King, R.J.; Siems, D.T. Isotopic and Trace-Element Constrants on Mantle and Crustal Contributions to Siberian Continental Flood basalts, Noril'sk Area, Siberia. Geochim. Cosmochim. Acta 1993, 57, 3677-3704. [CrossRef]

27. Petrov, O.V. (Ed.) Isotope Gecochemistry of the Noril'sk Deposits; VSEGEI: St-Peterbourg, Russia, 2017; p. 346. (In Russian)

28. Masaitis, V.L. Permian and Triassic Volcanism of Siberia: Problems of Dynamic Reconstructions. Zap. Vses. Miner. Ob-va 1983, 112, 412-425. (In Russian)

29. Malitch, N.S.; Grinson, A.S.; Tuganova, E.V.; Chernyshev, N.M. Rifting of the Siberian platform. In 28th Session of International Geological Congress. Tectonic Processes; Nauka: Moscow, Russia, 1988; pp. 184-193.

30. United States Geological Survey. Basalt, Hawaiian Volcanic Observatory, BHVO-2 Certificate of Analysis; United States Geological Survey: Reston, VA, USA, 1998. Available online: http:/ / minerals.cr.usgs.gov/geo_chem_ stand/basaltbhvo2.html (accessed on 17 January 2019).

31. Zolotukhin, V.V.; Vilensky, A.M.; Dyuzhikov, O.A. Basalts of the Siberian Platform; Nauka: Novosibirsk, Russia, 1986; p. 289. (In Russian)

32. Erinchek, Y.M.; Milshtein, E.D.; Kolesnik, N.N. Deep structure and geodynamic of the Siberia Platform kimberlite area. Reg. Geol. Metall. 2000, 10, 209-228. (In Russian)

33. Kuzmichev, A.B.; Danukalova, M.K. Central-Taimyr belt in Precambrian: Passive margin of the Siberian continent in Paleoproterozoic and active margin in Neoroterozoic. In Problems of Tectonic and Geodynamic of the Crust and Mantle; GEOS: Moscow, Russia, 2018; Volume 1, pp. 352-355. (In Russian)

34. Zolotukhin, V.V.; Vasil'ev, Y.R.; Dyuzhikov, O.A. Diversity of Traps and Initial Magmas: A Case of the Siberian Platform; Nauka: Novosibirsk, Russia, 1978; p. 289. (In Russian)

35. Zolotukhin, V.V.; Al'mukhamedov, A.I. Basalts of the Siberian platform: Composition and mechanism of formation. In Traps of Siberia and Deccan: Similarities and Differences; Nauka: Novosibirsk, Russia, 1991; pp. 7-39. (In Russian)

36. Kalvitsky, M.B. (Ed.) Geological Map of the Deposits of the Krasnoyarsk District, Khakasia and Tyva Republics, 1:1500000 Scale; Krasnoyarsk, Russia, 2005. (In Russian)

37. Lightfoot, P.C.; Naldrett, A.J.; Gorbachev, N.S.; Doherty, W.; Fedorenko, V.A. Geochemistry of the Siberian Trap of the Noril'sk Area, USSR, with Implications for the Relative Contributions of Crust and Mantle to Flood Basalt Magmatism. Contrib. Miner. Pet. 1990, 104, 631-644. [CrossRef]

38. Lightfoot, P.C.; Howkesworth, C.J.; Hergt, J.; Naldrett, A.J.; Gorbachev, N.S.; Fedorenko, V.A. Remobilisation of the continental lithosphere by a mantle plume: Major-, trace-element, and $\mathrm{Sr}-, \mathrm{Nd}-$, and Pb-isotopic evidence from picritic and tholeiitic lavas of the Noril'sk District, Siberian Trap, Russia. Contrib. Miner. Pet. 1993, 114, 171-188. [CrossRef]

39. Lightfoot, P.C.; Naldrett, A.J.; Gorbachev, N.S.; Fedorenko, V.A.; Howkesworth, C.G.; Hergt, J.; Doherty, W. Chemostratigraphy of Siberian Trap Lavas, Noril'sk District: Implication for the Source of Flood Basalt Magmas and Their Associated Ni-Cu Mineralization. In Proceedings of the Sudbury-Noril'sk Symposium; Ontario Geological Survey: Sudbury, ON, Canada, 1994; Volume 5, pp. 283-312.

40. Fedorenko, V.A.; Czamanske, G. Results of New Field and Geochemical Studies of the Volcanic Rocks of the Maymecha-Kotuy Area, Siberian Flood-Basalt Province, Russia. Intern. Geol. Rev. 1997, 39, 479-531. [CrossRef]

41. Arndt, N.T.; Chauvel, C.; Czamanske, G.; Fedorenko, V.A. Two Mantle Sources, Two Plumbing Systems: Tholeiitic and Alkaline Magmatism of the Maymecha River Basin, Siberian Flood Volcanic Province. Contrib. Miner. Pet. 1998, 133, 279-313. [CrossRef]

42. Sukhanova, E.N. Zoning of orebodies, intrusions, and tectonomagmatic clusters and its applied implications. In Geology and Mineral Resources of the Noril'sk Mining District; NTO Tsvetmet: Noril'sk, Russia, 1968; pp. 139-142. (In Russian) 
43. Dodin, D.A.; Batuev, B.N. Geology and petrology of the Talhakh differentiated intrusions and their metamorphic aureole. In Petrology and Ore Resource Potential of the Talnakh and Noril'sk Differentiated Intrusions; Nedra: Leningrad, Russia, 1971; pp. 31-100. (In Russian)

44. Zolotukhin, V.V.; Ryabov, V.V.; Vasil'ev, Y.R.; Shatkov, V.A. Petrology of the Talnakh Ore-Bearing Differentiated Trap Intrusion; Nauka: Novosibirsk, Russia, 1975; p. 378. (In Russian)

45. Likhachev, A.P. Ore-bearing Intrusions of the Noril'sk Region. In Proceeding of the Sudbury-Noril'sk Symposium; Ontario Geological Survey: Sudbury, ON, Canada, 1994; Volume 5, pp. 185-202.

46. Zen'ko, T.E.; Czamanske, G.K. Special and petrologic aspects of the Noril'sk and Talnakh ore junctions. In Proceeding of the Sudbury-Noril'sk Symposium; Lightfoot, P.C., Naldrett, A.J., Eds.; Ontario Geological Survey: Sudbury, ON, Canada, 1994; Volume 5, pp. 263-282.

47. Masaitis, V.L.; Egorov, L.S.; Ledneva, V.P. Proterozoic, Paleozoic and Mesozoic magmatic complexes of the Siberian platform. In Correlation of Magmatic and Metamorphic Complexes of the Eastern Siberia; VSEGEI: Leningrad, Russia, 1977; pp. 5-16. (In Russian)

48. Zolotukhin, V.V.; Vilensky, A.M.; Vasil'ev, Y.R. Magnesium Basic Rocks of Western Part of the Siberian Platform and Problems of Nickel Potential; Nauka: Novosibirsk, Russia, 1984; p. 208. (In Russian)

49. Oleynikov, B.V.; Tomshin, M.D. Evolution of basic intrusive magmatism of the Siberian platform in time. In Traps of Siberia and Deccan: Similarities and Differences; Nauka: Novosibirsk, Russia, 1991; pp. $39-63$. (In Russian)

50. Feoktistov, G.D. Late Paleozoic-Early Mesozoic intrusive traps of the Siberian Platform. In Traps of Siberia and Deccan: Similarities and Differences; Nauka: Novosibirsk, Russia, 1991; pp. 63-73. (In Russian)

51. Urvantsev, N.N. Noril'sk coal basin. Izv. Sib. Geolcom. 1921, 1, 12-17. (In Russian)

52. Urvantsev, N.N. (Ed.) North Siberian Ni-bearing area. Geol. Geophys. 1974, 3, 3-11. (In Russian)

53. Al'mukhamedov, A.I.; Medvedev, A.Y.; Zolotukhin, V.V. Evolutions of Permo-Triassic basalts of the Siberian Platform in time and space. Petrology 2004, 12, 339-353.

54. Fetisova, A.M.; Veselovskiy, R.V.; Latyshev, A.V.; Rad'ko, V.A.; Pavlov, V.E. Magnetic Stratigraphy of the Permian-Triassic Traps in the Kotuy River Valley (Siberian Platform). New Paleomagnetic Data. Stratigr. Geol. Correl. 2014, 22, 377-390. [CrossRef]

55. Sherman, M.L. (Ed.) Geological Map 1:50000 Scale of the Central part of the Noril'sk District; Ministry of Geology: Moscow, Russia, 1981. (In Russian)

56. Lyul'ko, V.A. (Ed.) Legend for 1: 50000 Scale Map, Noril'sk Group; Geoinformmark: Moscow, Russia, 1993 ; p. 53. (In Russian)

57. Distler, V.V.; Kunilov, V.E. Geology and Ore Deposits of the Noril'sk District; Moscow-Noril'sk, Russia, 1994; p. 43.

58. Al'mukhamedov, A.I.; Medvedev, A.Y.; Kirda, N.P. Comparing analyses of geodynamics of Permian -Triassic magmatism. Rus. Geol. Geophys. 1999, 40, 1575-1587.

59. Fedorenko, V.A.; Lightfoot, P.C.; Naldrett, A.J. Petrogenesis of the Siberian flood-basalt sequence at Noril'sk, north central Siberia. Intern. Geol. Rev. 1996, 38, 99-135. [CrossRef]

60. Dodin, D.A.; Batuev, B.N.; Mitenkov, G.A. Atlas of the Rocks and Ores of the Noril'sk Copper-Nickel Deposits; Nedra: Leningrad, Russia, 1971; p. 567. (In Russian)

61. Nesterenko, G.V.; Avilova, N.S.; Smirnova, N.P. Rare elements in traps of the Siberian Platform. Geokhimiya 1964, 10, 1015-1021. (In Russian)

62. Nesterenko, G.V.; Tikhonenkov, P.I.; Romashova, T.V. Basalts of plateau Putorana. Geokhimiya 1991, 10, 1419-1425. (In Russian)

63. Legends to the Geological Maps of 1:1000000 Scale, the 3-d Generation, Q-45-47, P-46-48, O-46-48. Available online: http:/ / www.vsegei.ru/ru/info/pub_ggk1000-3/ (accessed on 17 January 2019 ).

64. Sherman, M.L. (Ed.) Geological Map of the Noril'sk Ore District, 1:200000 Scale; VSEGEI: Leningrad, Russia, 1991.

65. Stekhin, A.S. Mineralogical and geochemical characteristics of the Cu-Ni ores of the Oktyabr'skoe and Talnakh deposits. In Proceeding of the Sudbury-Noril'sk Symposium; Ontario Geological Survey: Sudbury, ON, Canada, 1994; Volume 5, pp. 217-230.

66. Torgashin, A.S. Geology of the massive and copper ores of the western part of the oktyabr'skoe deposit. In Proceeding of the Sudbury-Noril'sk Symposium; Ontario Geological Survey: Sudbury, ON, Canada, 1994; Volume 5, pp. 231-242. 
67. Krivolutskaya, N.A.; Gongalsky, B.I. Dyumtaleysky massif, Taimyr, Russia: A magmatic derivative from a complex mantle source. In Mineral Research for a High-Tech World; SGA: Uppsala, Sweeden, 2013; Volume 3, pp. 1036-1040.

68. Krivolutskaya, N.A.; Gongalsky, B.I.; Kedrovskaya, T.B.; Kubrakova, I.V.; Tyutyunnik, O.A.; Chikatueva, V.Y.; Bychkova, Y.V.; Magazina, L.; Kovalchuk, E.N.; Yakushev, A.I.; et al. Geology of the Western Flanks of the Oktyabr'skoe Deposit, Noril'sk District, Russia: Evidence of a Closed Magmatic System. Miner. Dep. 2018. [CrossRef]

69. Malitch, K.N.; Badanina, I.Y.; Romanov, A.P.; Sluzhenikin, S.F. U-Pb age and Hf-Nd-Sr-Cu-S isotope systematics of the Binyuda and Dyumtaley ore-bearing intrusions (Taimyr, Russia). Lithosphera 2016, 1, 107-128. (In Russian)

70. Malitch, K.N.; Belousova, E.A.; Griffin, W.L.; Badanina, I.Y.; Latypov, R.M.; Sluzhenikin, S.F. New insights on the origin of ultramafic-mafic intrusions and associated Ni-Cu-PGE sulfide deposits of the Noril'sk and Taimyr provinces, Russia: Evidence from radiogenic and stable isotope data. In Processes and Ore Deposits of Ultramafic-Mafic Magmas through Space and Time; Elsevier: New York City, NY, USA, 2017; Volume 7, pp. 198-237.

71. Tomshin, M.D.; Kopylova, A.G.; Vasilyeva, A.E. Geochemical and isotope characteristics of intrusive traps in the Eastern Siberian Platform. In Proceedings of the 14th International Multidisciplinary Scientific GeoConference \& EXPO SGEM2014, Albena, Bulgaria, 17-26 June 2014; Volume 1, pp. 113-120. (In Russian)

72. Krivolutskaya, N.; Tolstykh, N.; Kedrovskaya, T.; Naumov, K.; Kubrakova, I.; Tutunnik, O.; Gongalsky, B.; Kovalchuk, E.; Magazina, L.; Bychkova, Y.; et al. World-class PGE-Cu-Ni Talnakh Deposit: New Data on the Structure and Unique Mineralization of the South-Western Branch. Minerals 2018, 8, 124. [CrossRef]

73. Genkin, A.D.; Evstigneeva, T.L. Associations of platinum-group minerals of the Noril'sk copper-nickel sulfide ores. Econ. Geol. 1986, 81, 1203-1212. [CrossRef]

74. Genkin, A.D.; Distler, V.V.; Gladyshev, G.D.; Filimonova, A.A.; Evstigneeva, T.L.; Kovalenker, V.A.; Laputina, I.P.; Smirnov, A.V.; Grokhovskaya, T.L. Sulfide Copper-Nickel Ores of the Noril'sk Deposits; Nauka: Moscow, Russia, 1981; 281p. (In Russian)

75. Distler, V.V. Platinum mineralization of the Noril'sk deposits. In Proceeding of the Sudbury-Noril'skSymposium; Ontario Geological Survey: Sudbury, ON, Canada, 1994; pp. 243-262.

76. Sluzhenikin, S.F. Platinum-copper-nickel and platinum ores of the Norilsk region and their ore mineralization. Russ. J. Gen. Chem. 2011, 81, 1288-1301. (In Russian) [CrossRef]

77. Krivolutskaya, N.; Gongalsky, B.; Dolgal, A.; Svirskaya, N.; Vekshina, T. Siberian Traps in the Noril'sk Area: A Corrected Scheme of Magmatism Evolution. IOP Conf. Ser. Earth Environ. Sci. 2016, 44, 1-6. [CrossRef]

78. Krivolutskaya, N.A.; Kuzmin, D.V.; Gongalsky, B.I.; Rostshina, I.A.; Kononkova, N.N.; Svirskaya, N.M.; Romashova, T.V. Stages of trap magmatism in the Norilsk area: New data on the structure and geochemistry of the volcanic rocks. Geochem. Intern. 2018, 56, 419-437. [CrossRef]

79. Heunemann, C.; Krasa, D.; Soffel, H.; Gurevitch, E.; Bachtadse, V. Directions and intensities of the Earth's magnetic field during a reversal: Results from the Permo-Triassic Siberian trap basalts, Russia. Earth Plan. Sci. Lett. 2004, 218, 197-213. [CrossRef]

80. Pavlov, V.; Fluteau, F.; Veselovskiy, R.; Fetisova, A.; Latyshev, A.; Elkins-Tanton, L.T.; Sobolev, A.V.; Krivolutskaya, N.A. Volcanic pulses in the Siberian Traps as inferred from Permo-Triassic geomagnetic secular variations. In Volcanism and Global Environmental Change; Schmidt, A., Ed.; Cambridge University Press: Cambridge, UK, 2015; pp. 63-78.

81. Hawkesworth, C.J.; Lightfoot, P.C.; Fedorenko, V.A.; Blake, S.; Naldrett, A.J.; Doherty, W.; Gorbachev, N.S. Magma differentiation and mineralization in the Siberian flood basalts. Lithos 1995, 34, 61-88. [CrossRef]

82. Gurevich, E.; Westphal, M.; Daragan-Suchov, J.; Feinberg, H.; Pozzi, J.P.; Khramov, A.N. Paleomagnetism and magnetostratigraphy of the traps from the Western Taimyr (northern Siberia) and the Permo-Triassic crisis. Earth Plan. Sci. Lett. 1995, 136, 461-473. [CrossRef]

83. Walderhaug, H.J.; Eide, E.A.; Scott, R.A.; Inger, S.; Golionko, E.G. Paleomagnetism and 40Ar/39Ar geochronology from the South Taimyr igneous complex, Arctic Russia: A Middle-Late Triassic magmatic pulse after Siberian flood-basalt volcanism. Geophys. J. Int. 2005, 163, 501-517. [CrossRef]

84. Reichow, M.K.; Saunders, A.D.; Scott, R.A.; Millar, I.L.; Barfod, D.; Pringle, M.S.; Rogers, N.W.; Hammond, S. Petrogenesis and timing of mafic magmatism, South Taimyr, Arctic Siberia: A northerly continuation of the Siberian Traps. Lithos 2016, 248, 382-401. [CrossRef] 
85. Shcherbakova, V.V.; Zhidkov, G.V.; Shcherbakov, V.P.; Latyshev, A.V.; Fetisova, A.M. Verifying the Mesozoic Dipole Low Hypothesis by the Siberian Trap Data. Izv. Phys. Solid Earth 2015, 51, 362-382. [CrossRef]

86. Sobolev, A.V.; Krivolutskaya, N.A.; Kuzmin, D.V. Petrology of the Parental Melts and Mantle Sources of Siberian Trap Magmatism. Petrology 2009, 17, 253-286. [CrossRef]

87. Sobolev, A.V.; Slutsky, A.B. Composition and Crystallization Conditions of the Parental Melt of the Siberian Meymechites in Connection with the General Problem of Ultrabasic Magmas. Geol. Geophys. 1984, 12, 97-110. (In Russian)

88. Sobolev, A.V.; Sobolev, S.V.; Kuzmin, D.V.; Malitch, K.N.; Petrunin, A.G. Siberian meimechites: Origin and relation to flood basalts and kimberlites. Rus. Geol. Geophys. 2009, 50, 999-1033. [CrossRef]

89. Latyshev, A.V.; Veselovsky, R.V.; Ivanov, A.V. Paleomagnetism of the Permian-Triassic intrusions from the Tunguska syncline and the Angara-Taseeva depression Siberian Traps Large Igneous Province: Evidence of contrasting styles of magmatism. Tectonophysics 2018. [CrossRef]

90. GEOROCK Database. Available online: http://georoc.mpch-mainz.gwdg.de/georoc (accessed on 17 January 2019).

91. Hofmann, A.W. Chemical differentiation of the Earth: The relationship between mantle, continental crust, and oceanic crust. Earth Plan. Sci. Lett. 1988, 90, 297-314. [CrossRef]

92. Lind, E.N.; Kropotov, S.V.; Czamanske, G.K.; Gromme, S.C.; Fedorenko, V.A. Paleomagnetism of Siberian flood basalts of the Noril'sk area: A constraint on eruption duration. Intern. Geol. Rev. 1994, 36, 1139-1151. [CrossRef]

93. Badanina, I.Y.; Malitch, K.N.; Romanov, A.P. Isotopic-Geochemical Characteristics of the Ore-Bearing Ultramafic-Mafic Intrusions of Western Taimyr, Russia. Dokl. Earth Sci. 2014, 458, 1165-1167. [CrossRef]

94. Romanov, A.P.; Kurbatov, I.I.; Malitch, K.N.; Snisar, S.G.; Borodina, E.V.; Erykalov, S.P. The Resource Potential of the Platinum Metals in Western Taimyr. Platin. Russ. 2011, 7, 135-160. (In Russian)

95. Irvine, T.N.; Baragar, W.R.A. A guide to the chemical classification of the common volcanic rocks. Can. J. Earth Sci. 1971, 8, 523-548. [CrossRef]

96. Latyshev, A.V.; Veselovsky, R.V.; Ivanov, A.V.; Fetisova, A.V.; Pavlov, V.E. Short Intense Bursts in Magmatic Activity in the South of Siberian Platform (Angara-Taseeva Depression): The Paleomagnetic Evidence. Izv. Phys. Solid Earth 2013, 49, 823-835. [CrossRef]

97. Proskurnin, V.F.; Vernikovsky, V.A.; Metelkin, D.V.; Petrushkov, B.S.; Vernikovskaya, A.E.; Gavrish, A.V.; Bagaeva, A.A.; Matushkin, N.Y.; Vinogradova, N.P.; Larionov, A.N. The rhiolite-granite association in the central Taimyr zone: Evidence of accretionary-collisional events in the Neoproterozoic. Rus. Geol. Geophys. 2014, 55, 23-40. [CrossRef]

98. Afanasenkov, A.P.; Nikishin, A.M.; Unger, A.V.; Bordunov, S.I.; Lugovaya, O.V.; Chikishev, A.A.; Yakovishina, E.V. The tectonics and stages of the geological history of the Yenisei-Khatanga Basin and the conjugate Taimyr Orogen. Geotectonics 2016, 50, 161-178. [CrossRef]

99. Dolgal, A.S.; Realization, V.N. Strakhov ideas in interpretation of geopotential fields. In Academician V.N. Strakhov as Geophysics and Mathematic; Nauka: Moscow, Russia, 2012; pp. 55-78. (In Russian)

100. Balk, P.I.; Dolgal, A.S.; Pugin, A.V.; Michurin, A.V.; Simanov, A.A.; Sharkhimullin, A.F. Effective algorithms for sourcewise approximation of geopotential fields. Izv. Phys. Solid Earth 2016, 52, 896-911. [CrossRef]

101. Baranov, V.A. A new method for interpretation of aeromagnetic maps: Psevdo-gravimetric anomalies. Geophysics 1957, 22, 359-383. [CrossRef]

102. Kazais, V.I. Seismic-gravity-magnetic modeling of the platform structures of the northern part of the Siberian platform. Geol. Oil Gas 1997, 11, 30-37. (In Russian)

103. Tuganova, E.V. Criteria for predicting of complex sulfide platinum-copper-nickel deposits. In Natural Resources of Taimyr; URR for TAO: Dudinka, Russia, 2004; Volume 2, pp. 65-84. (In Russian)

104. Dobretsov, N.L.; Vernikovsky, V.A. Mantle Plumes and Their Geologic Manifestations. Intern. Geol. Rev. 2001, 43, 771-787. [CrossRef]

105. Dobretsov, N.L.; Borisenko, A.S.; Izokh, A.E.; Zhmodik, S.M. A termochemical model of Eurasian Permo-Triassic mantle plumes as a basis for prediction and exploration for $\mathrm{Cu}-\mathrm{Ni}-\mathrm{PGE}$ rare metals ore deposits. Rus. Geol. Geophys. 2010, 51, 1159-1187. [CrossRef]

106. Dobretsov, N.L.; Borisenko, A.S.; Izokh, A.E. Termochemical deep mantle plums as a source of ore potencial on the planet. Nauka Pervykh Ruk 2011, 6, 37-43. (In Russian) 
107. Kuzmin, M.I.; Yarmolyuk, V.V. Mantle plums in Northeastern Asia and their role in the formation of endogenous deposits. Rus. Geol. Geophys. 2014, 2, 153-184.

108. Brügmann, G.E.; Naldrett, A.J.; Asif, M.; Lightfoot, P.C.; Gorbachev, N.S.; Fedorenko, V.A. Siderophile and chalcophile metals as tracers of the evolution of the Siberian trap in the Noril'sk region, Russia. Geochim. Cosmochim. Acta 1993, 57, 2001-2018. [CrossRef]

109. Lightfoot, P.C.; Keays, R.R. Siderophile and chalcophile metal variations in flood basalts from the Siberian Trap, Noril'sk Region: Implications for the origin of the Ni-Cu-PGE sulfide ores. Econ. Geol. 2005, 100, 439-462. [CrossRef]

110. Keays, R.R.; Lightfoot, P.C. Crustal sulfur is required to form magmatic Ni-Cu sulfide deposits: Evidence from chalcophile element signatures of Siberian and Deccan Trap basalts. Miner. Depos. 2010, 45, 241-257. [CrossRef]

111. Keays, R.R.; Lightfoot, P.C. Siderophile and chalcophile metal variations in Tertiary picrites and basalts from West Greenland with implications for the sulfide saturation history of continental flood basalt magmas. Miner. Depos. 2007, 42, 319-336. [CrossRef]

112. Lightfoot, P.C.; Hawkesworth, C.J. Flood basalts and magmatic Ni, Cu and PGE sulfide mineralization: Comparative geochemistry of the Noril'sk (Siberian Traps) and West Greenland sequences. Geophys. Monogr. Am. Geophys. Union 1997, 100, 357-380.

113. Lightfoot, P.C.; Hawkesworth, C.J.; Olshefsky, K.; Green, A.H.; Doherty, W.; Keays, R.R. Geochemistry of Tertiary tholeiites and picrites from Qeqertarssuaq (Disko Island) and the Nuussuaq Peninsula, West Greenland with implications for the mineral potential of comagmatic intrusions. Contrib. Miner. Pet. 1997, 128, 139-163. [CrossRef]

114. Begg, G.C.; Hronsky, J.A.M.; Arndt, N.T.; Griffin, W.L.; O’Reilly, S.Y.; Hayward, N. Lithospheric, Cratonic, and Geodynamic Setting of Ni-Cu-PGE Sulfide Deposits. Econ. Geol. 2010, 105, 1057-1070. [CrossRef]

115. Zotov, I.A. Transmagmatic Fluids in Magmatism and Ore Formation; Nauka: Moscow, Russia, 1989; p. 213. (In Russian)

116. Grinenko, L.N. Sources of sulfur of the nickeliferous and barren gabbrodolerite intrusions of the northwest Siberian platform. Intern. Geol. Rev. 1985, 27, 695-708. [CrossRef]

117. Li, C.; Naldrett, A.J.; Shmitt, A.K. Magmatic anhydrite-Sulfide assemblages in plumbing system of the Siberian Traps. Geology 2009, 37, 259-262. [CrossRef]

118. Distler, V.V.; Grokhovskaya, T.L.; Evstigneeva, T.L.; Sluzhenikin, S.F.; Filimonova, A.A.; Dyuzhikov, O.A. Petrology of Magmatic Sulfide Ore Formation; Nauka: Moscow, Russia, 1988; p. 232. (In Russian)

119. Krivolutskaya, N.A.; Sobolev, A.V.; Snisar, S.G.; Gongalskiy, B.I.; Hauff, B.; KuzminM, D.V.; Tushentsova, I.N.; Svirskaya, N.M.; Kononkova, N.N.; Schlychkova, T.B. Mineralogy, geochemistry and stratigraphy of the Maslovsky Pt-Cu-Ni sulfide deposit, Noril'sk Region, Russia: Implications for relationship of ore-bearing intrusions and lavas. Miner. Depos. 2012, 47,69-88. [CrossRef]

120. Ripley, E.M.; Lightfoot, P.C.; Li, C.; Elswick, E.R. Sulfur isotopic studies of continental flood basalts in the Noril'sk region: Implications for the association between lavas and ore-bearing intrusion. Geochim. Cosmochim. Acta 2003, 67, 2805-2817. [CrossRef]

121. Krivolutskaya, N.A.; Plechova, A.A.; Kostitsyn, Y.A.; Belyatsky, B.V.; Roshchina, I.A.; Svirskaya, N.M.; Kononkova, N.N. Geochemical aspects of the assimilation of host rocks by basalt during the formation of Noril'sk Cu-Ni ores. Petrology 2014, 22, 128-150. [CrossRef]

(C) 2019 by the authors. Licensee MDPI, Basel, Switzerland. This article is an open access article distributed under the terms and conditions of the Creative Commons Attribution (CC BY) license (http://creativecommons.org/licenses/by/4.0/). 